\title{
Improved existence for the characteristic initial value problem with the conformal Einstein field equations
}

\author{
David Hilditch ${ }^{1}$. Juan A. Valiente Kroon ${ }^{2}$ (D) Peng Zhao ${ }^{2}$
}

Received: 30 June 2020 / Accepted: 26 August 2020 / Published online: 5 September 2020

(c) The Author(s) 2020

\begin{abstract}
We adapt Luk's analysis of the characteristic initial value problem in general relativity to the asymptotic characteristic problem for the conformal Einstein field equations to demonstrate the local existence of solutions in a neighbourhood of the set on which the data are given. In particular, we obtain existence of solutions along a narrow rectangle along null infinity which, in turn, corresponds to an infinite domain in the asymptotic region of the physical spacetime. This result generalises work by Kánnár on the local existence of solutions to the characteristic initial value problem by means of Rendall's reduction strategy. In analysing the conformal Einstein equations we make use of the Newman-Penrose formalism and a gauge due to J. Stewart.
\end{abstract}

Keywords Characteristic initial value problem - Conformal Einstein field equations . Newman-Penrose formalism · Asymptotically simple spacetimes

\section{Contents}

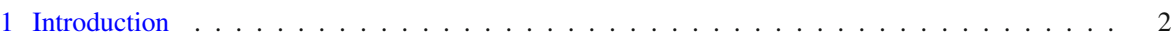

New insights .............................. 4

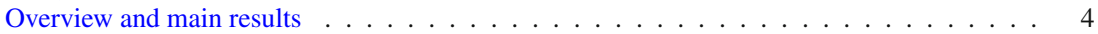

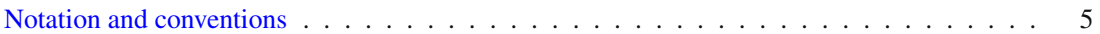

2 The vacuum conformal Einstein field equations . . . . . . . . . . . . . . . . . . 5

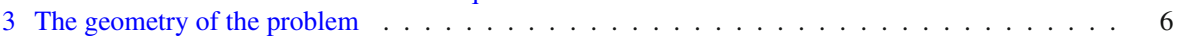

$凶 \quad$ Juan A. Valiente Kroon

j.a.valiente-kroon@qmul.ac.uk

David Hilditch

david.hilditch@tecnico.ulisboa.pt

Peng Zhao

p.zhao@qmul.ac.uk

1 CENTRA, Departamento de Física, Instituto Superior Técnico IST, Universidade de Lisboa UL, Avenida Rovisco Pais 1, 1049 Lisbon, Portugal

2 School of Mathematical Sciences, Queen Mary, University of London, Mile End Road, London E1 $4 \mathrm{NS}, \mathrm{UK}$ 
3.1 Basic setting . . . . . . . . . . . . . . . . . . . . . . 7

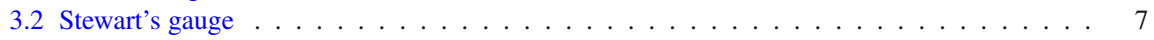

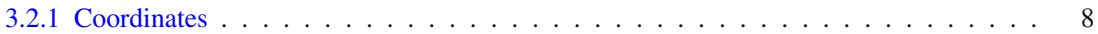

3.2 The NP frame . . . . . . . . . . . . . . . . . . . . . . . . . 8

3.2 .3 Spin connection coefficients . . . . . . . . . . . . . . . . . . . . . 10

3.2 .4 Equations for the frame coefficients . . . . . . . . . . . . . . . . . 10

3.2 .5 The conformal gauge conditions . . . . . . . . . . . . . . . . . 11

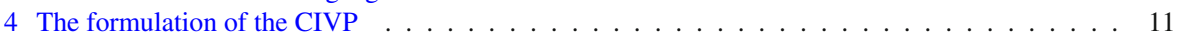

4.1 Specifiable free data . . . . . . . . . . . . . . . . . . . . . . . . . . . . . . . . . . . . 11

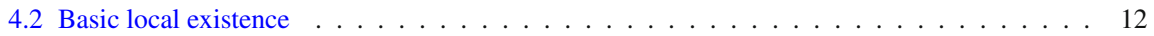

4.2.1 The reduced conformal field equations . . . . . . . . . . . . . . . . 12

4.2.2 Computation of the formal derivatives on $\mathcal{N}_{\star}^{\prime} \cup \mathscr{I}^{-}$and propagation of the constraints 15

4.2 .3 Summary . . . . . . . . . . . . . . . . . . . . . . 16

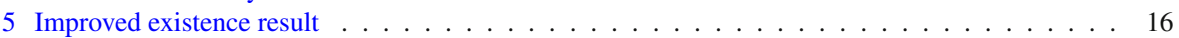

5.1 Integration identities and definitions of norms . . . . . . . . . . . . . . . 16

5.2 Estimates for the components of the frames and the conformal factor . . . . . . . . . . . 17

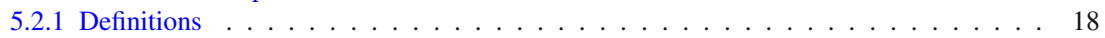

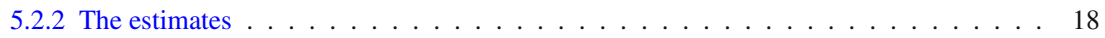

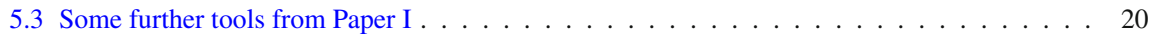

5.3 .1 General estimates for transport equations . . . . . . . . . . . . . . . . . . . . . . . . . . . . . . . . 20

5.3 .2 Grönwall-type inequalities . . . . . . . . . . . . . . . . . . . . 21

5.3 .3 Sobolev inequalities . . . . . . . . . . . . . . . . . . . . 22

6 Main estimates . . . . . . . . . . . . . . . . . . . . . . . . . 22

6.1 Norms . . . . . . . . . . . . . . . . . . . . . . 23

6.2 Estimates for the connection coefficients and the derivative of conformal factor . . . . . . 24

6.3 First estimates for the curvature . . . . . . . . . . . . . . . . . . . . . . . . . . . . . . . . . .

6.4 The energy estimates for the curvature . . . . . . . . . . . . . . . . . . . . . . . . . . . . . . . . . .

6.4.1 Analysis of the rescaled Weyl tensor . . . . . . . . . . . . . . . . . . . . . . . . . . . . . . . . . . . 45

6.4 .2 Analysis of the Ricci curvature . . . . . . . . . . . . . . . . . . . . . . . . . . . . . . . . . . . . . . . . . . . . .

7 Concluding the argument . . . . . . . . . . . . . . . . . . . . 53

A The conformal field equations in the NP formalism . . . . . . . . . . . . . . . . . . . 54

A.1 The NP field equations . . . . . . . . . . . . . . . . . . . . . . . . . . . . . . . . . . 54

A.2 Conformal vacuum Einstein field equations . . . . . . . . . . . . . . . . . . . . . . . . . . . . . . . . . . . 55

A.2.1 The first conformal Einstein field equation . . . . . . . . . . . . . . . . . . . . . 56

A.2.2 The second conformal Einstein field equation . . . . . . . . . . . . . . . . 56

A.2.3 The third conformal Einstein field equation . . . . . . . . . . . . . . . . . 56

A.2.4 The fourth conformal Einstein field equation . . . . . . . . . . . . . . . 57

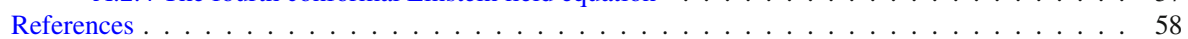

\section{Introduction}

This article is the second of a series in which we study the characteristic initial value problem (CIVP) in General Relativity in a range of settings. In [1] (henceforth Paper I) it has been shown that Luk's strategy - see [2], to obtain an improved local existence result for the characteristic problem for the vacuum Einstein field equations can be adapted to a different gauge based on the Newman-Penrose (NP) formalism, the Stewart gauge, see [3,4]. In the present article we consider the asymptotic characteristic initial value problem, a CIVP for Friedrich's conformal Einstein field equations (a regular conformal representation of the vacuum Einstein field equations) in which one of the null initial hypersurfaces is a portion of past null infinity, and show that Luk's strategy can also be adapted to this setting. Accordingly, we obtain a domain of existence of the solution to the conformal Einstein field equations on a narrow rectangle 
(a)

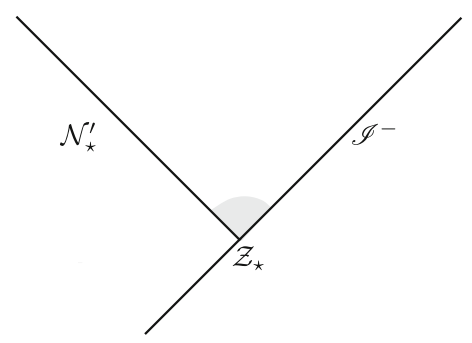

(b)

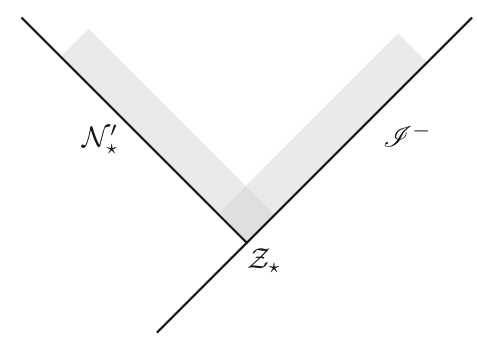

Fig. 1 Comparison of the existence domains for the characteristic problem: a existence domain using Rendall's strategy based on the reduction to a standard Cauchy problem; b existence domain using Luk's strategy - in principle, the long side of the rectangles extends for as much as one has control on the initial data

having a portion of null infinity as one of its long sides—-see Fig. 1. In doing so we improve Kánnár's local existence result for the asymptotic CIVP in which existence of a solution is only guaranteed in a neighbourhood of the intersection of the initial null hypersurfaces_-see [5] and also [6], Chapter 18. Expressed in terms of a solution to the Einstein field equations the improved rectangular existence domain corresponds, in fact, to an infinite domain. Kánnár's result is, in turn, an extension to the setting of smooth (i.e. $C^{\infty}$ ) functions of Friedrich's seminal analysis of the CIVP for the Einstein and conformal Einstein field equations in the analytic setting — see [7-9].

A different strategy to the proof of the main theorem in the present article has been given in [10]. This alternative proof makes use of the fact that the conformal Einstein field equations imply a set of quasilinear wave equations for which a general theory of improved existence of solutions to the characteristic problem had been developed. Thus, the approach used in the present equation provides a different, complementary insight into the structural properties of the conformal Einstein field equations.

An alternative asymptotic CIVP for the conformal Einstein field equations can be formulated by prescribing initial data on a cone representing past null infinity including its vertex-which corresponds to past timelike infinity. The setup of this CIVP was given in $[11,12]$. Statements about the existence of solutions to this problem have been obtained in $[13,14]$, see also [15]. These results also provide improved existence results - that is, the existence of solutions is not restricted to the tip of the cone of past null infinity but extends to a neighbourhood of the cone away from the vertex as long as there is control of the initial data. An important open problem in this respect is to obtain a solution which includes spatial infinity. The analysis in $[13,14]$ builds on the theory of the CIVP for characteristic cones developed in $[16,17]$. This theory makes use of systems of second order hyperbolic equations and is, in principle, different from Luk's approach.

The results in this article hold on portions of of null infinity which are away from the points $i^{0}$ and $i^{+}$representing, respectively, spatial and future timelike infinity. At these points both the conformal structure and the gauge used in the present analysis become singular. The extension of the analysis to include these points requires the 
construction of estimates which do not degerate at these caustics. In the case of spatial infinity, a tatalising possibility is the combination of the methods of the present article with the estimates for linear fields within the framework of the cylinder at spatial infinity developed in [18]. This analysis requires a detailed analysis of asymptotic expansions near spatial infinity.

\section{New insights}

The NP formalism has played an influential role in mathematical relativity. Nevertheless, its use in the formulation of existence results has been limited. The main motivations behind the analysis in this article is to understand the structural properties of the conformal Einstein field equations. In this spirit, we avoid the use of general theory as in [10] and give a detailed discussion of the various arguments to convince the reader that they indeed follow through. In order to keep the length of the article at bay, we focus primarily on those aspects of the analysis which are novel or not present in the discussion of Paper I. In this article we use the phrase improved existence result to mean an improvement on Kánnaár's existence result in a neighbourhood of the intersection of past null infinity and an incoming null cone. This improved result is, in a sense, optimal in that it provides existence in a neighbourhood of the initial hypersurfaces as long as one has control on the initial data see Fig. 1. Our main result is presented in Theorem 7 in Sect. 2. While the results of the present article have been stated, for conciseness with past null infinity and an incoming null cone as initial hypersurfaces, analogous results can be readily obtained, mutatis mutandi, for a setting where the data is prescribed on future null infinity and an outgoing light cone. In this case the development of the data is located in the past of the union of the initial null hypersurfaces.

\section{Overview and main results}

In Sect. 2 we start by providing a brief review of the main technical tool of this work: the conformal Einsten field equations and its relation to the vacuum Einstein field equations. In Sect. 3 we give a discussion of the geometric formulation of the asymptotic CIVP and the gauge choice behind this formulation-i.e. Stewart's gauge. Section 4 is concerned with the basic local existence theory of the asymptotic CIVP. In particular, we discuss the choices of freely specifiable initial data, the hyperbolic reduction procedure, the computation of formal derivatives and the propagation of the constraints and the gauge. The basic existence theorem is given in Theorem 1. Section 5 provides the basic setting for the formulation of the improved existence result. Section 6 discusses the construction of the estimates required to establish the improved existence result. Finally, Sect. 7 provides the precise statement of the main result of our analysis.

The article contains one appendix listing in explicit form the NP formulation of the conformal Einstein field equations used in the analysis. 


\section{Notation and conventions}

We take $\{a, b, c, \ldots\}$ to denote abstract tensor indices whereas $\{\mu, v, \lambda, \ldots\}$ will be used as spacetime coordinate indices with the values $0, \ldots, 3$. Our conventions for the curvature tensors are fixed by the relation

$$
\left(\nabla_{a} \nabla_{b}-\nabla_{b} \nabla_{a}\right) v^{c}=R_{d a b}^{c} v^{d}
$$

We make systematic use of the NP formalism as described, for example, in [19,20]. In particular, the signature of Lorentzian metrics is (+- - ). Many of our derivations, although straightforward, are fairly lengthy, so we have included in Appendix A a complete summary of the equations of the NP-formalism, highlighting the simplifications that occur with our particular gauge.

\section{The vacuum conformal Einstein field equations}

The purpose of this section is to provide a succinct summary of Friedrich's conformal Einstein field equations - see e.g. [8,21]. The reader interested in full details, derivation and discussion is referred to [6], Chapter 8. In this article we restrict our attention to the 4-dimensional vacuum case with vanishing Cosmological constant.

In what follows let $\left(\tilde{\mathcal{M}}, \tilde{g}_{a b}\right)$ denote a vacuum spacetime. Friedrich's conformal Einstein field equations are a regular conformal representation of the Einstein field equations. That is, they are equations for a conformally rescaled metric $g_{a b}=\Xi^{2} \tilde{g}_{a b}$, a conformal factor $\Xi$ and concommitants which are formally regular up to the conformal boundary, i.e. the points for which $\Xi=0$, and which imply, for $\Xi \neq 0$, a solution to the Einstein field equations. In the following, denote by $\left(\mathcal{M}, g_{a b}\right)$ the conformal extension of $\left(\tilde{\mathcal{M}}, \tilde{g}_{a b}\right)$ generated by the conformal factor $\Xi$. Following the usual terminology we refer to $\left(\tilde{\mathcal{M}}, \tilde{g}_{a b}\right)$ and $\left(\mathcal{M}, g_{a b}\right)$, respectively, as the physical and unphysical spacetimes. The sets of points of the conformal boundary giving rise to a hypersurface-i.e. the points for which $\mathbf{d} \Xi \neq 0$-are called null infinity, $\mathscr{I}$. In this article we assume, for simplicity, that $\mathscr{I}=\partial \mathcal{M}$. Spacetimes admitting this type of smooth conformal extension are known as asymptotically simple-see [6,19,22] for the classic precise definition. Null infinity can be shown to correspond to the endpoints of null geodesics and, thus, it consists of two disconnected components, past and future null infinity, $\mathscr{I}^{-}$and $\mathscr{I}^{+}$. In this article, for concreteness we restrict our discussion to a neighbourhood of $\mathscr{I}^{-}$.

In what follows let $\nabla_{a}$ denote the Levi-Civita connection of the metric $g_{a b}$ and let $R_{a b}, C^{a}{ }_{b c d}$ be its Ricci and Weyl tensors, respectively. In order to introduce the conformal Einstein field equations we further define the fields 


$$
\begin{aligned}
L_{a b} & \equiv \frac{1}{2} R_{a b}-\frac{1}{12} R g_{a b}, \\
d^{a}{ }_{b c d} & \equiv \Xi^{-1} C_{b c d}^{a}, \\
s & \equiv \frac{1}{4} \nabla^{a} \nabla_{a} \Xi+\frac{1}{24} R \Xi,
\end{aligned}
$$

the Schouten tensor, the rescaled Weyl tensor and the Friedrich scalar respectively. In terms of the latter the vacuum conformal Einstein field equations with vanishing Cosmological constant are given by

$$
\begin{aligned}
\nabla_{a} \nabla_{b} \Xi & =-\Xi L_{a b}+s g_{a b}, \\
\nabla_{a} s & =-L_{a c} \nabla^{c} \Xi, \\
\nabla_{c} L_{d b}-\nabla_{d} L_{c b} & =\nabla_{a} \Xi d^{a}{ }_{b c d}, \\
\nabla_{a} d^{a}{ }_{b c d} & =0, \\
6 \Xi s-3 \nabla_{c} \Xi \nabla^{c} \Xi & =0, \\
R_{d a b}^{c} & =C_{d a b}^{c}+2\left(\delta^{c}{ }_{[a} L_{b] d}-g_{d[a} L_{b]}^{c}\right) .
\end{aligned}
$$

More details can be found in [6]. In Appendix A.2 we give the spinorial counterpart of these equations and their components equations in Newman-Penrose (NP) frame. This spinorial formulation of the equations will be used systematically in the rest of the article. It is just recalled that the spinoral version of the equations follows directly by suitably contracting the tensorial equations with the soldering forms—see e.g. [6] for more details.

The relation between the conformal Einstein field Eqs. (2a)-(2f) and the Einstein field equations is expressed in the following result:

Proposition 1 (solutions of the conformal vacuum Einstein field equations as solutions to the vacuum Einstein field equations) Let

$$
\left(g_{a b}, \Xi, s, L_{a b}, d^{a}{ }_{b c d}\right)
$$

denote a solution to Eqs. (2a)-(2d) and (2f) such that $\Xi \neq 0$ on an open set $\mathcal{U} \subset \mathcal{M}$. If, in addition, Eq. (2e) is satisfied at a point $p \in \mathcal{U}$, then the metric $\tilde{g}_{a b}=\Xi^{-2} g_{a b}$ is a solution to the vacuum Einstein field equations on $\mathcal{U}$.

The proof can be found in Chapter 8 of [6].

\section{The geometry of the problem}

In this section, we will discuss the geometric and the gauge choices in the asymptotic CIVP on past null infinity. 


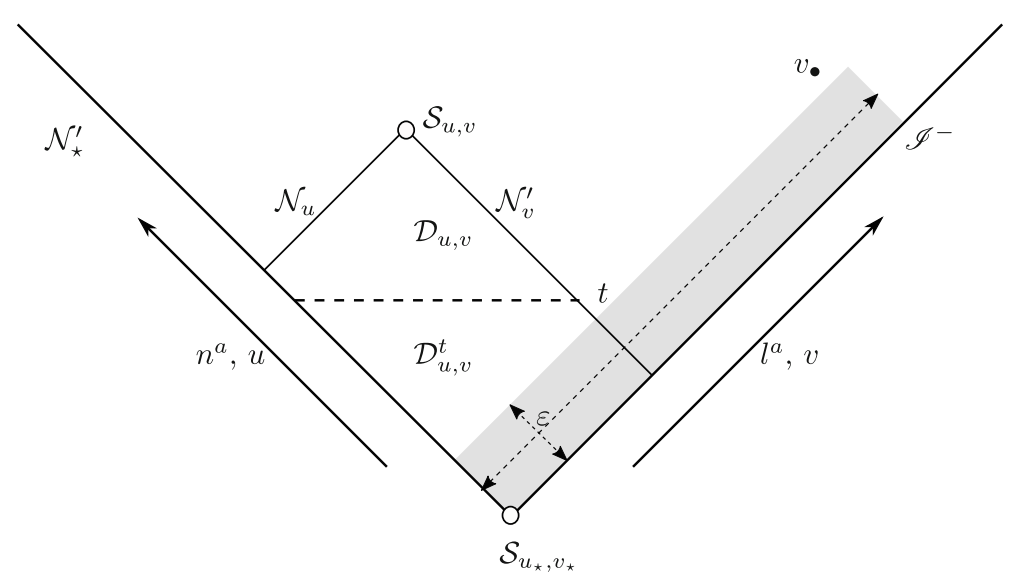

Fig. 2 Setup for Stewart's gauge. The construction makes use of a double null foliation of the future domain of dependence of the initial hypersurface $\mathscr{I}^{-} \cup \mathcal{N}_{\star}^{\prime}$. The coordinates and NP null tetrad are adapted to this geometric setting. The analysis in this article is focused on the arbitrarily thin grey rectangular domain along the hypersurface $\mathscr{I}^{-}$. The argument can be adapted, in a suitable manner, to a similar rectangle along $\mathcal{N}_{\star}^{\prime}$. See the main text for the definitions of the various regions and objects

\subsection{Basic setting}

Our basic geometric setting consists of an unphysical manifold $\mathcal{M}$ with a boundary and an edge. The boundary consists of two null hypersurfaces: $\mathscr{I}^{-}$, past null infinity on which $\Xi=0$; and $\mathcal{N}_{\star}^{\prime}$, an incoming null hypersurface with non-vacuum intersection $\mathcal{S}_{\star} \equiv \mathscr{I}^{-} \cap \mathcal{N}_{\star}^{\prime}$. We will assume that $\mathcal{S}_{\star} \approx \mathbb{S}^{2}$. In a neighbourhood $\mathcal{U}$ of $\mathcal{S}_{\star}$, one can introduce coordinates $x=\left(x^{\mu}\right)$ with $x^{0}=v$ and $x^{1}=u$ such that, at least in a neighbourhood of $\mathcal{S}_{\star}$ one can write

$$
\mathscr{I}^{-}=\{p \in \mathcal{U} \mid u(p)=0\}, \quad \mathcal{N}_{\star}^{\prime}=\{p \in \mathcal{U} \mid v(p)=0\}
$$

Given suitable data on $\left(\mathscr{I}^{-} \cup \mathcal{N}_{\star}^{\prime}\right) \cap \mathcal{U}$ one is interested in making statements about the existence and uniqueness of solutions to the conformal Einstein field equations on some open set

$$
\mathcal{V} \subset\{p \in \mathcal{U} \mid u(p) \geq 0, v(p) \geq 0\}
$$

which we identify with a subset of the future domain of dependence, $D^{+}\left(\mathscr{I}^{-} \cup \mathcal{N}_{\star}^{\prime}\right)$, of $\mathscr{I}^{-} \cup \mathcal{N}_{\star}^{\prime}$.

\subsection{Stewart's gauge}

The basic geometric setting described in the previous section is supplemented by a gauge choice first introduced by Stewart [19]. 


\subsubsection{Coordinates}

It is convenient to regard the 2-dimensional surface $\mathcal{S}_{\star}$ as a submanifold of a spacelike hypersurface $S$. The subsequent discussion will be restricted to the future of the hypersurface. As $\mathcal{S}_{\star} \approx \mathbb{S}^{2}$, one has that $\mathcal{S}_{\star}$ divides the spacelike hypersurface into two regions, the interior of $\mathcal{S}_{\star}$ and the exterior of $\mathcal{S}_{\star}$. Now, consider a foliation of the spacelike hypersurface by 2 -dimensional surfaces with the topology of $\mathbb{S}^{2}$ which includes $\mathcal{S}_{\star}$. At each of the 2-dimensional surfaces we assume there pass two null hypersurfaces. Further, we assume that one of these hypersurfaces has the property that the projection of the tangent vectors of their generators at $S$ point outwards. We call these null hypersurfaces outgoing light cones. Moreover, it is also assumed that one of these hypersurfaces has the property that the projection of the tangent vectors of their generators at $\mathcal{S}_{\star}$ point inwards. We call these null hypersurfaces ingoing light cones.

Thus, at least locally, one obtains a 1-parameter family of outgoing null hypersurfaces $\mathcal{N}_{u}$ and a 1-parameter family of ingoing null hypersurfaces $\mathcal{N}_{v}^{\prime}$. One can then define scalar fields $u$ and $v$ by the requirements, respectively, that $u$ is constant on each of the $\mathcal{N}_{u}$ and $v$ is constant on each $\mathcal{N}_{v}^{\prime}$. In particular, we assume that $\mathcal{N}_{0}=\mathscr{I}^{-}$and $\mathcal{N}_{0}^{\prime}=\mathcal{N}_{\star}^{\prime}$. Following standard usage, we call $u$ a retarded time and $v$ an advanced time. The scalar fields $u$ and $v$ will be used as coordinates in a neighbourhood of $\mathcal{S}_{\star}$. To complete the coordinate system, consider arbitrary coordinates $\left(x^{\mathcal{A}}\right)$ on $\mathcal{S}_{\star}$, with the index $\mathcal{A}^{\mathcal{A}}$ taking the values 2,3 . These coordinates are then propagated into $\mathscr{I}^{-}$by requiring them to be constant along the generators of $\mathscr{I}^{-}$. Once coordinates have been defined on $\mathscr{I}^{-}$, one can propagate them into $\mathcal{V}$ by requiring them to be constant along the generators of each $\mathcal{N}_{v}^{\prime}$. In this manner one obtains a coordinate system $\left(x^{\mu}\right)=\left(v, u, x^{\mathcal{A}}\right)$ in $\mathcal{V}$.

We use the notation $\mathcal{N}_{u}\left(v_{1}, v_{2}\right)$ to denote the part of the hypersurface $\mathcal{N}_{u}$ with $v_{1} \leq v \leq v_{2}$. Likewise $\mathcal{N}_{v}^{\prime}\left(u_{1}, u_{2}\right)$ has a similar definition. We denote the sphere intersected by $\mathcal{N}_{u}$ and $\mathcal{N}_{v}^{\prime}$ by $\mathcal{S}_{u, v}$. We define the region

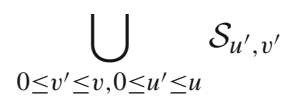

as $\mathcal{D}_{u, v}$. We also define the time function $t \equiv u+v$, and the truncated causal diamond,

$$
\mathcal{D}_{u, v}^{\tilde{t}} \equiv \mathcal{D}_{u, v} \cap\{t \leq \tilde{t}\} .
$$

Remark 1 It is observed that while the null coordinte $u$ has a compact range, this is, in principle, not the case for $v$.

\subsubsection{The NP frame}

A null Newman-Penrose (NP) tetrad is constructed by choosing vector fields $l^{a}$ and $n^{a}$ tangent to the generators of $\mathcal{N}_{u}$ and $\mathcal{N}_{v}^{\prime}$ respectively. Following the standard conventions we make use of the normalisation $g_{a b} l^{a} n^{b}=1$ which is preserved under boost transformations. This freedom can be used to set $n_{a}=\nabla_{a} v$. This requirement still 
leaves some freedom left as one can choose a relabelling of the form $v \mapsto V(v)$. Next, we choose the vector fields $m^{a}$ and $\bar{m}^{a}$ so that they are tangent to the surfaces $\mathcal{S}_{u, v} \equiv \mathcal{N}_{u} \cap \mathcal{N}_{v}^{\prime}$ and satisfy the conditions $g_{a b} m^{a} \bar{m}^{b}=-1, g_{a b} m^{a} m^{b}=0$. This leaves the freedom to perform a spin transformation at each point.

Now, observing that, by construction, on the generators of each null hypersurface $\mathcal{N}_{v}^{\prime}$ only the coordinate $u$ varies, one has that

$$
n^{\mu} \partial_{\mu}=Q \partial_{u}
$$

where $Q$ is a real function of the position. Further, since the vector $l^{a}$ is tangent to the generators of each $\mathcal{N}_{u}$ and $l^{a} n_{a}=l^{a} \nabla_{a} v=1$, one has that

$$
l^{\mu} \boldsymbol{\partial}_{\mu}=\boldsymbol{\partial}_{v}+C^{\mathcal{A}} \boldsymbol{\partial}_{\mathcal{A}}
$$

where, again, the components $C^{\mathcal{A}}$ are real functions of the position. By construction, the coordinates $\left(x^{\mathcal{A}}\right)$ do not vary along the generators of $\mathscr{I}^{-}$, that is, one has that $l^{a} \nabla_{a} x^{\mathcal{A}}=0$. Accordingly, one has

$$
C^{\mathcal{A}}=0 \text { on } \mathscr{I}^{-} \text {. }
$$

Finally, since $m^{a}$ and $\bar{m}^{a}$ span the tangent space of each surface $\mathcal{S}_{u, v}$ one has that

$$
m^{\mu} \boldsymbol{\partial}_{\mu}=P^{\mathcal{A}} \boldsymbol{\partial}_{\mathcal{A}}
$$

where the coefficients $P^{\mathcal{A}}$ are complex functions.

Summarising, we make the following assumption:

Assumption 1 On $\mathcal{V}$ one can find a Newman-Penrose frame $\left\{l^{a}, n^{a}, m^{a}, \bar{m}^{a}\right\}$ of the form:

$$
\begin{aligned}
\boldsymbol{l} & =\boldsymbol{\partial}_{v}+C^{\mathcal{A}} \boldsymbol{\partial}_{\mathcal{A}}, \\
\boldsymbol{n} & =Q \boldsymbol{\partial}_{u}, \\
\boldsymbol{m} & =P^{\mathcal{A}_{\boldsymbol{A}}} \boldsymbol{\partial}_{\mathcal{A}} .
\end{aligned}
$$

Remark 2 In view of the normalisation condition $g_{a b} m^{a} \bar{m}^{b}=-1$, there are only 3 independent real functions in the coefficients $P^{\mathcal{A}}$. Thus, $Q, C^{\mathcal{A}}$ together with $P^{\mathcal{A}}$ give six scalar fields describing the metric. The components $\left(g^{\mu v}\right)$ of the contravariant form of the metric $g_{a b}$ are of the form

$$
\left(g^{\mu \nu}\right)=\left(\begin{array}{ccc}
0 & Q & 0 \\
Q & 0 & Q C^{\mathcal{A}} \\
0 & Q C^{\mathcal{A}} & \sigma^{\mathcal{A B}}
\end{array}\right)
$$

where

$$
\sigma^{\mathcal{A B}} \equiv-\left(P^{\mathcal{A}} \overline{P^{\mathcal{B}}}+\overline{P^{\mathcal{A}}} P^{\mathcal{B}}\right)
$$

is the (contravariant) induced metric on $\mathcal{S}_{u, v}$. 
On $\mathcal{N}_{\star}^{\prime}$ one has that $\boldsymbol{n}=Q \boldsymbol{\partial}_{u}$. As the coordinates $\left(x^{\mathcal{A}}\right)$ are constant along the generators of $\mathscr{I}^{-}$and $\mathcal{N}_{\star}^{\prime}$, it follows that on $\mathcal{N}_{\star}^{\prime}$ the coefficient $Q$ is only a function of $u$. Thus, without loss of generality one can reparameterise $u$ so as to set $Q=1$ on $\mathcal{N}_{\star}^{\prime}$.

\subsubsection{Spin connection coefficients}

Direct inspection of the NP commutators (12a)-(12d) applied to the coordinates $\left(v, u, x^{2}, x^{3}\right)$ taking into account together with the remaining gauge freedom in the vector frame of Assumption 10 leads to the following:

Lemma 1 The NP frame of Assumption 10 can be chosen such that

$$
\begin{aligned}
& \kappa=v=\gamma=0, \\
& \rho=\bar{\rho}, \quad \mu=\bar{\mu}, \\
& \pi=\alpha+\bar{\beta},
\end{aligned}
$$

on $\mathcal{V}$ and, furthermore, with

$$
\epsilon-\bar{\epsilon}=0 \text { on } \mathcal{V} \cap \mathscr{I}^{-} \text {. }
$$

Proof The proof of this result is analogous to that of Lemma 1 in Paper I.

\subsubsection{Equations for the frame coefficients}

Taking into account the conditions of the spin connection coefficients given by (5a)$(5 \mathrm{c})$, the remaining commutators yield the equations

$$
\begin{aligned}
\Delta C^{\mathcal{A}} & =-(\bar{\tau}+\pi) P^{\mathcal{A}}-(\tau+\bar{\pi}) \bar{P}^{\mathcal{A}}, \\
\Delta P^{\mathcal{A}} & =-\mu P^{\mathcal{A}}-\bar{\lambda} \bar{P}^{\mathcal{A}}, \\
D P^{\mathcal{A}}-\delta C^{\mathcal{A}} & =(\bar{\rho}+\epsilon-\bar{\epsilon}) P^{\mathcal{A}}+\sigma \bar{P}^{\mathcal{A}}, \\
D Q & =-(\epsilon+\bar{\epsilon}) Q, \\
\bar{\delta} P^{\mathcal{A}}-\delta \bar{P}^{\mathcal{A}} & =(\alpha-\bar{\beta}) P^{\mathcal{A}}-(\bar{\alpha}-\beta) \bar{P}^{\mathcal{A}}, \\
\delta Q & =(\tau-\bar{\pi}) Q .
\end{aligned}
$$

Equations (6a)-(6b) allow the evolution of the frame coefficients $C^{\mathcal{A}}$ and $P^{\mathcal{A}}$ off of the null hypersurface $\mathcal{N}_{\star}^{\prime}$. Equations (6c)-(6d) allow the coefficients $Q$ and $P^{\mathcal{A}}$ to be evolved along the null generators of $\mathscr{I}^{-}$. Finally, (6e)-(6f) provide constraints for $Q$ and $P^{\mathcal{A}}$ on the spheres $\mathcal{S}_{u, v}$. 


\subsubsection{The conformal gauge conditions}

The conformal Einstein field equations have an in-built conformal freedom which can be exploited to simplify the geometric setting of the problem. This freedom allows us, in particular, to select in an indirect manner the conformal factor via a conformal gauge source function. As it is customary in the NP formalism let $\Lambda$ denote the Ricci scalar divided by 24 . One has the following:

Lemma 2 (conformal gauge conditions) Let $(\tilde{\mathcal{M}}, \tilde{\boldsymbol{g}})$ denote an asymptotically simple spacetime satisfying Ric $[\tilde{\boldsymbol{g}}]=0$ and let $(\mathcal{M}, \boldsymbol{g}, \boldsymbol{\Xi})$ with $\boldsymbol{g}=\Xi^{2} \tilde{\boldsymbol{g}}$ be a conformal extension for which the condition $\Xi=0$ describes past null infinity $\mathscr{I}^{-}$. Given the previous NP frame (4a)-(4c), the conformal factor $\Xi$ can be chosen so that given a null hypersurface $\mathcal{N}_{\star}^{\prime}$ intersecting $\mathscr{I}^{-}$on $\mathcal{S}_{\star} \approx \mathbb{S}^{2}$ one has

$$
\Lambda=-\frac{1}{24} R(x), \quad \text { in a neighourhood } \mathcal{V} \text { of } \mathcal{S}_{\star} \text { on } J^{+}\left(\mathcal{S}_{\star}\right)
$$

where $R(x)$ is an arbitrary function of the coordinates. Moreover, one has the additional gauge conditions

$$
\begin{aligned}
& \Sigma_{2}=1, \quad \mu=\rho=0 \text { on } \mathcal{S}_{\star}, \\
& \Phi_{22}=0 \quad \text { on } \mathcal{N}_{\star}^{\prime}, \\
& \Phi_{00}=0 \quad \text { on } \mathscr{I}^{-} .
\end{aligned}
$$

Proof The proof of the above result is analogous to that of Lemma 18.2 in [6].

\section{The formulation of the CIVP}

In this section we analyse general aspects of the asymptotic CIVP for the conformal vacuum Einstein field equations with data on the null hypersurface $\mathscr{I}^{-}$and $\mathcal{N}_{\star}^{\prime}$. A key feature of the setting is the existence of a hierarchical structure in the reduced conformal equations which allows to identify the basic reduced initial data set from which the full initial data on $\mathscr{I}^{-} \cup \mathcal{N}_{\star}^{\prime}$ for the conformal Einstein field equations can be computed.

\subsection{Specifiable free data}

The following result identifies a possible choice of freely specifiable initial data for the asymptotic CIVP:

Lemma 3 (freely specifiable data for the characteristic problem) Assume that the gauge condition given by Lemmas 1 and 2 is satisfied in a neighbourhood $\mathcal{V}$ of $\mathcal{S}_{\star}$. Initial data for the conformal Einstein field equations on $\mathscr{I}^{-} \cup \mathcal{N}_{\star}^{\prime}$ can be computed from the reduced data set $\mathbf{r}_{\star}$ consisting of: 


$$
\begin{aligned}
& \phi_{0}, \epsilon+\bar{\epsilon} \text { on } \mathscr{I}^{-}, \\
& \phi_{4} \text { on } \mathcal{N}_{\star}^{\prime}, \\
& \lambda, \phi_{2}+\bar{\phi}_{2}, \Phi_{20}, \phi_{3}, \quad P^{\mathcal{A}}, \text { on } \mathcal{S}_{\star} .
\end{aligned}
$$

Remark 3 An alternative, less symmetric, reduced initial data set is given by:

$$
\begin{aligned}
& \lambda, \epsilon+\bar{\epsilon} \text { on } \mathscr{I}^{-}, \\
& \phi_{4} \text { on } \mathcal{N}_{\star}^{\prime}, \\
& \phi_{3}, \phi_{2}+\bar{\phi}_{2}, P^{\mathcal{A}}, \text { on } \mathcal{S}_{\star} .
\end{aligned}
$$

Proof The proof of this result follows from a lengthy but straightforward computation on the same lines of Lemma 2 in Paper I. See also Section 18.2 in [5,6].

Remark 4 From the smoothness of the freely specifiable component $\phi_{4}$ on the incoming null hypersurface $\mathcal{N}_{\star}^{\prime}$ and, in particular at the intersection with $\mathscr{I}^{-}$it follows that that the resulting spacetime will satisfy the peeling behaviour near past null infinitysee e.g. [6], Chapter 10. A reformulation of our characteristic problem to future null infinity, for which now $\phi_{0}$ is freely specifiable data along an outgoing null hypersurface, gives rise mutatis mutandi to solutions with peeling at $\mathscr{I}^{+}$.

\subsection{Basic local existence}

To apply the theory of CIVP, as discussed say in Section 12.5 of [6], one has to extract a suitable symmetric hyperbolic evolution system out of the conformal field equations and the structure equations. The gauge introduced in Sect. 3.2 allows us to perform this reduction.

\subsubsection{The reduced conformal field equations}

In what follows, we group the four directional derivatives of the conformal factor and $s$ as

$$
\Sigma^{t} \equiv\left(\Sigma_{1}, \Sigma_{2}, \Sigma_{3}, \Sigma_{4}, s\right)
$$

the components of the frame as

$$
\boldsymbol{e}^{t} \equiv\left(C^{\mathcal{A}}, P^{\mathcal{A}}, Q\right)
$$

the spin connection coefficients not fixed by the gauge as

$$
\Gamma^{t} \equiv(\epsilon, \pi, \beta, \mu, \alpha, \lambda, \tau, \sigma, \rho),
$$

the independent components of the rescaled Weyl spinor as

$$
\boldsymbol{\phi}^{t} \equiv\left(\phi_{0}, \phi_{1}, \phi_{2}, \phi_{3}, \phi_{4}\right)
$$


and finally those of tracefree Ricci spinor as

$$
\boldsymbol{\Phi}^{t} \equiv\left(\Phi_{00}, \Phi_{01}, \Phi_{11}, \Phi_{02}, \Phi_{12}, \Phi_{22}\right)
$$

where ${ }^{t}$ denotes the operation of taking the transpose of a column vector.

A suitable symmetric hyperbolic system for the four directional derivatives of the conformal factor, the frame components and the spin coefficients can be obtained from Eqs. (15d)-(15f), (15f)*, (17b), (6a), (6b), (6d) and (13a)-(13d), (13f), (13g), (13k), $(13 \mathrm{~m}),(13 \mathrm{o})$, respectively. These can be written in the schematic form,

$$
\begin{aligned}
\mathcal{D}_{0} \Sigma & =\boldsymbol{B}_{0}(\boldsymbol{\Sigma}, \boldsymbol{\Gamma}, s) \boldsymbol{\Sigma}, \\
\mathcal{D}_{1} \boldsymbol{e} & =\boldsymbol{B}_{1}(\boldsymbol{\Gamma}, \boldsymbol{e}) \boldsymbol{e}, \\
\mathcal{D}_{2} \boldsymbol{\Gamma} & =\boldsymbol{B}_{2}(\boldsymbol{\Gamma}, \boldsymbol{\phi}, \boldsymbol{\Phi}) \boldsymbol{\Gamma},
\end{aligned}
$$

where $\mathcal{D}_{0}, \mathcal{D}_{1}$ and $\mathcal{D}_{2}$ are given by

$$
\begin{aligned}
& \mathcal{D}_{0} \equiv \operatorname{diag}(\Delta, \Delta, \Delta, \Delta, \Delta), \\
& \mathcal{D}_{1} \equiv \operatorname{diag}(\Delta, \Delta, D), \\
& \mathcal{D}_{2} \equiv \operatorname{diag}(\Delta, \Delta, \Delta, \Delta, \Delta, \Delta, D, D, D),
\end{aligned}
$$

and $\boldsymbol{B}_{0}, \boldsymbol{B}_{1}, \boldsymbol{B}_{2}$ are smooth matrix-valued functions of their arguments, whose explicit form will not be required.

The components of the third conformal field Eq. (2c), Eqs. (19a)-(19m) can be reorganised as

$$
\mathcal{D}_{3} \Phi=B_{3} \Phi
$$

where

$$
\mathcal{D}_{3}=\left(\begin{array}{cccccc}
\Delta & -\bar{\delta} & 0 & 0 & 0 & 0 \\
-\delta & D+2 \Delta & -\delta & -\bar{\delta} & 0 & 0 \\
0 & -\bar{\delta} & D+\Delta & 0 & -\bar{\delta} & 0 \\
0 & -\delta & 0 & D+\Delta & -\delta & 0 \\
0 & 0 & -\delta & -\bar{\delta} & 2 D+\Delta & -\delta \\
0 & 0 & 0 & 0 & -\bar{\delta} & D
\end{array}\right)
$$

with $\boldsymbol{B}_{\mathbf{3}}=\boldsymbol{B}_{\mathbf{3}}\left(\Phi, \phi, \Gamma, \Sigma_{i}\right)$. Writing

$$
\mathcal{D}_{3}=A_{3}^{\mu} \partial_{\mu}
$$

one has that

$$
\begin{aligned}
& \boldsymbol{A}_{3}^{v}=\operatorname{diag}(0,1,1,1,2,1), \\
& \boldsymbol{A}_{3}^{u}=\operatorname{diag}(Q, 2 Q, Q, Q, Q, 0) .
\end{aligned}
$$


and

$$
A_{3}^{\mathcal{A}}=\left(\begin{array}{cccccc}
0 & -\bar{P}^{\mathcal{A}} & 0 & 0 & 0 & 0 \\
-P^{\mathcal{A}} & C^{\mathcal{A}} & -P^{\mathcal{A}} & -\bar{P}^{\mathcal{A}} & 0 & 0 \\
0 & -\bar{P}^{\mathcal{A}} & C^{\mathcal{A}} & 0 & -\bar{P}^{\mathcal{A}} & 0 \\
0 & -P^{\mathcal{A}} & 0 & C^{\mathcal{A}} & -P^{\mathcal{A}} & 0 \\
0 & 0 & -P^{\mathcal{A}} & -\bar{P}^{\mathcal{A}} & 2 C^{\mathcal{A}} & -P^{\mathcal{A}} \\
0 & 0 & 0 & 0 & -\bar{P}^{\mathcal{A}} & C^{\mathcal{A}}
\end{array}\right)
$$

To be specific, the equations above are obtained through the combinations $(19 \mathrm{a})+(19 \mathrm{k})$, $(19 j)+2(19 b)+(191),(19 d) *+(19 h)^{*},(19 c)+(19 i),(19 e)+2(19 g)+(19 l)$ and $(19 f)+(19 m)$, respectively. It can be readily verified that the matrices $\boldsymbol{A}_{3}^{\mu}$ are Hermitian. Moreover,

$$
\boldsymbol{A}_{3}^{\mu}\left(l_{\mu}+n_{\mu}\right)=\operatorname{diag}(1,3,2,2,3,1)
$$

is likewise clearly positive definite.

The components of the fourth conformal Eqs. (2d), (21a)-(21h), can be grouped as

$$
\mathcal{D}_{4} \phi=\boldsymbol{B}_{4} \phi
$$

where

$$
\mathcal{D}_{4}=\left(\begin{array}{ccccc}
\Delta & -\delta & 0 & 0 & 0 \\
-\bar{\delta} & D+\Delta & -\delta & 0 & 0 \\
0 & -\bar{\delta} & D+\Delta & -\delta & 0 \\
0 & 0 & -\bar{\delta} & D+\Delta & -\delta \\
0 & 0 & 0 & -\bar{\delta} & D
\end{array}\right)
$$

and $\boldsymbol{B}_{4}=\boldsymbol{B}_{4}(\boldsymbol{\phi}, \boldsymbol{\Gamma})$. Again, writing

$$
\mathcal{D}_{4}=\boldsymbol{A}_{4}^{\mu} \partial_{\mu},
$$

one has that

$$
\begin{aligned}
& \boldsymbol{A}_{4}^{v}=\operatorname{diag}(0,1,1,1,1), \\
& \boldsymbol{A}_{4}^{u}=\operatorname{diag}(Q, Q, Q, Q, 0),
\end{aligned}
$$

and

$$
\boldsymbol{A}_{4}^{\mathcal{A}}=\left(\begin{array}{ccccc}
0 & -P^{\mathcal{A}} & 0 & 0 & 0 \\
-\bar{P}^{\mathcal{A}} & C^{\mathcal{A}} & -P^{\mathcal{A}} & 0 & 0 \\
0 & -\bar{P}^{\mathcal{A}} & C^{\mathcal{A}} & -P^{\mathcal{A}} & 0 \\
0 & 0 & -\bar{P}^{\mathcal{A}} & C^{\mathcal{A}} & -P^{\mathcal{A}} \\
0 & 0 & 0 & -\bar{P}^{\mathcal{A}} & C^{\mathcal{A}}
\end{array}\right)
$$


Specifically, the above matricial expressions are obtained from the combinations (21a), $(21 \mathrm{~b})+(21 \mathrm{e}),(21 \mathrm{c})+(21 \mathrm{f}),(21 \mathrm{~d})+(21 \mathrm{~g})$ and (21h). Again, the matrices $\boldsymbol{A}_{4}^{\mu}$ can be seen to be Hermitian and, moreover, one has that

$$
\boldsymbol{A}_{4}^{\mu}\left(l_{\mu}+n_{\mu}\right)=\operatorname{diag}(1,2,2,2,1)
$$

is clearly positive definite.

We can summarise the above discussion as:

Lemma 4 The evolution system

$$
\begin{aligned}
& \mathcal{D}_{0} \Sigma=B_{0} \Sigma \\
& \mathcal{D}_{1} \boldsymbol{e}=\boldsymbol{B}_{1} \boldsymbol{e}, \\
& \mathcal{D}_{2} \Gamma=\boldsymbol{B}_{2} \Gamma, \\
& \mathcal{D}_{3} \boldsymbol{\Phi}=\boldsymbol{B}_{3} \boldsymbol{\Phi}, \\
& \mathcal{D}_{4} \boldsymbol{\phi}=\boldsymbol{B}_{4} \boldsymbol{\phi},
\end{aligned}
$$

is symmetric hyperbolic with respect to the direction given by

$$
\tau^{a}=l^{a}+n^{a}
$$

\subsubsection{Computation of the formal derivatives on $\mathcal{N}_{\star}^{\prime} \cup \mathscr{I}^{-}$and propagation of the constraints}

As discussed in Section 12.5 of [6], the existence and uniqueness of solutions to a CIVP can be obtained via an auxiliary Cauchy problem on the spacelike hypersurface

$$
S \equiv\left\{p \in \mathbb{R} \times \mathbb{R} \times \mathbb{S}^{2} \mid v(p)+u(p)=0\right\} .
$$

The formulation of this problem depends crucially on Whitney's extension theorem, which requires being able to evaluate all derivatives (interior and transverse) of initial data on $\mathcal{N}_{\star}^{\prime} \cup \mathscr{I}^{-}$. One has the following:

Lemma 5 (computation of formal derivatives) Any arbitrary formal derivatives of the unknown functions $\{\boldsymbol{\Sigma}, \boldsymbol{e}, \boldsymbol{\Gamma}, \boldsymbol{\Phi}, \boldsymbol{\phi}\}$ on $\mathcal{N}_{\star}^{\prime} \cup \mathscr{I}^{-}$can be computed from the prescribed initial data $\boldsymbol{r}_{\star}$ for the reduced conformal field equations on $\mathcal{N}_{\star}^{\prime} \cup \mathscr{I}^{-}$.

Proof The statement follows from a careful inspection of the conformal field equations in the present gauge, see Section 18.2 in [5,6] for more details.

Moreover, using arguments similar to those discussed in [6], Section 12.5, one can establish the following result concerning the relation between the reduced equations and the full conformal vacuum Einstein field equations:

Proposition 2 (propagation of the constraints) A solution of the reduced conformal field Eqs. (8a)-(8e) on a neighbourhood $\mathcal{V}$ of $\mathcal{S}_{\star}$ on $J^{+}\left(\mathcal{S}_{\star}\right)$ that coincides with initial data on $\mathcal{N}_{\star}^{\prime} \cup \mathscr{I}^{-}$satisfying the conformal equations gives rise to a solution of the conformal Einstein field Eqs. (2a)-(2f) on $\mathcal{V}$. 
In addition, one has that:

Corollary 1 (preservation of the conformal gauge) Let $\boldsymbol{u}$ denote a solution to the characteristic problem for the conformal field equations on a neighbourhood $\mathcal{V}$ of $\mathcal{S}_{\star}$ on $\mathrm{J}^{+}\left(\mathcal{S}_{\star}\right)$ which satisfies the gauge conditions given in Lemmas 1 and 2. Then the metric $\boldsymbol{g}$ constructed from the components of the solution $\boldsymbol{u}$ satisfies the conformal vacuum Einstein field equations in a gauge for which $R[\boldsymbol{g}]=R(x)$.

\subsubsection{Summary}

Combining the analysis above and applying the theory of the CIVP for the symmetric hyperbolic systems of Section 12.5 of [6], one obtains the following existence result:

Theorem 1 (existence and uniqueness to the standard asymptotic characteristic problem) Given a smooth reduced initial data set $\boldsymbol{r}_{\star}$ for the conformal Einstein field equations on $\mathcal{N}_{\star}^{\prime} \cup \mathscr{I}^{-}$, there exists a unique smooth solution of the conformal field equations in a neighbourhood $\mathcal{V}$ of $\mathcal{S}_{\star}$ on $J^{+}\left(\mathcal{S}_{\star}\right)$ which implies the prescribed initial data on $\mathcal{N}_{\star}^{\prime} \cup \mathscr{I}^{-}$. Moreover, this solution to the conformal Einstein field equations implies, in turn, a solution to the vacuum Einstein field equations in a neighbourhood of past null infinity.

Remark 5 Although the region $\mathcal{V}$ is, in the unphysical picture, finite, from the physical point of view, it corresponds to an infinite domain of the asymptotic region near past null infinity.

\section{Improved existence result}

In this section we provide the basic setting for the improved local existence result for the asymptotic CIVP for the conformal Einstein field equations using Luk's method. Our analysis builds on the general formalism developed in Paper I. Accordingly, in order to avoid tedious repetition we focus our attention on the novel aspects and specific challenges raised by the conformal field equations. Thus, results and definitions already appearing in Paper I are stated without proofs and where appropriate we quote results directly.

The main difference between the present analysis and that of Paper I is that when dealing with the conformal Einstein field equations one has more unknown equations to take care of. Specifically, we now have the conformal factor, its derivatives and the components of the tracefree Ricci tensor.

\subsection{Integration identities and definitions of norms}

In this section we recall some of the basic definitions introduced in Paper I.

Integration In what follows let $\phi$ denote a scalar field. We define the integration on $\mathcal{S}_{u, v}$ as

$$
\int_{\mathcal{S}_{u, v}} \phi \equiv \int_{\mathcal{S}_{u, v}} \phi \mathrm{d} \sigma,
$$


where $\mathrm{d} \sigma \equiv \sqrt{|\operatorname{det} \sigma|} \mathrm{d} x^{2} \mathrm{~d} x^{3}$ denotes the volume element of the induced metric $\sigma$ on $\mathcal{S}_{u, v}$. On the truncated causal diamonds $\mathcal{D}_{u, v}^{t}$, we define integration using the volume form of the spacetime metric as follows:

$$
\begin{aligned}
\int_{\mathcal{D}_{u, v}^{t}} \phi & \equiv \int_{0}^{u} \int_{0}^{\tilde{v}} \int_{\mathcal{S}_{u, v}} \phi \sqrt{|\operatorname{det} \boldsymbol{g}|} \mathrm{d} x^{2} \mathrm{~d} x^{3} \mathrm{~d} v \mathrm{~d} u \\
& =\int_{0}^{u} \int_{0}^{\tilde{v}} \int_{\mathcal{S}_{u, v}} Q^{-1} \phi \sqrt{|\operatorname{det} \sigma|} \mathrm{d} x^{2} \mathrm{~d} x^{3} \mathrm{~d} v \mathrm{~d} u
\end{aligned}
$$

with $\tilde{v}=\min (v, t-u)$. Integration in the full causal diamond is denoted in the obvious way with omission of the label $t$. As there are no canonical volume forms on $\mathcal{N}_{u}$ and $\mathcal{N}_{v}^{\prime}$, we define integration on these null hypersurfaces by

$$
\begin{aligned}
\int_{\mathcal{N}_{u}(0, v)} \phi & \equiv \int_{0}^{v} \int_{\mathcal{S}_{u, v}} \phi \sqrt{|\operatorname{det} \sigma|} \mathrm{d} x^{2} \mathrm{~d} x^{3} \mathrm{~d} v^{\prime}, \\
\int_{\mathcal{N}_{v}^{\prime}(0, u)} \phi & \equiv \int_{0}^{u} \int_{\mathcal{S}_{u, v}} \phi \sqrt{|\operatorname{det} \sigma|} \mathrm{d} x^{2} \mathrm{~d} x^{3} \mathrm{~d} u^{\prime} .
\end{aligned}
$$

We also define

$$
\int_{\mathcal{N}_{u}^{t}} \phi \equiv \int_{\mathcal{N}_{u}\left(I^{t}\right)} \phi, \quad \int_{\mathcal{N}_{v}^{\prime} t} \phi \equiv \int_{\mathcal{N}_{v}^{\prime}[0, \varepsilon]^{t}} \phi
$$

where $I^{t} \equiv\left[0, \min \left(v_{\bullet}, t-u\right)\right]$ for $v_{\bullet} \in \mathbb{R}^{+}$denotes the truncated long integration interval, and $[0, \varepsilon]^{t} \equiv[0, \min (\varepsilon, t-v)]$ the truncated short integration interval.

Norms For $1 \leq p<\infty$, we define the following $L^{p}$-type norms:

$$
\begin{aligned}
\|\phi\|_{L^{p}\left(\mathcal{S}_{u, v}\right)} & \equiv\left(\int_{\mathcal{S}_{u, v}}|\phi|^{p}\right)^{1 / p}, \quad\|\phi\|_{L^{p}\left(\mathcal{N}_{u}^{t}\right)} \equiv\left(\int_{\mathcal{N}_{u}^{t}}|\phi|^{p}\right)^{1 / p}, \\
\|\phi\|_{L^{p}\left(\mathcal{N}_{v}^{\prime} t\right)} & \equiv\left(\int_{\mathcal{N}_{v}^{\prime} t}|\phi|^{p}\right)^{1 / p} .
\end{aligned}
$$

The corresponding $L^{\infty}$ norm is defined by

$$
\|\phi\|_{L^{\infty}\left(\mathcal{S}_{u, v}\right)} \equiv \sup _{\mathcal{S}_{u, v}}|\phi|
$$

\subsection{Estimates for the components of the frames and the conformal factor}

A first step in the analysis in Paper I was the construction of basic estimates for the components of the frame in terms of the initial conditions. A similar step is required for the conformal Einstein field equations. The main difference in this case is that one 
also needs to obtain some basic control on the conformal factors and its derivatives. These estimates are constructed presently.

\subsubsection{Definitions}

Following Paper I, in the following it will be convenient to define the following norm measuring the size of the initial value of the components of the frame:

$$
\Delta_{e_{\star}} \equiv \sup _{\mathscr{I}^{-}, \mathcal{N}_{\star}^{\prime}}\left(|Q|,\left|Q^{-1}\right|,\left|C^{\mathcal{A}}\right|,\left|P^{\mathcal{A}}\right|\right) .
$$

Moreover, we define a scalar

$$
\chi \equiv \Delta \log Q
$$

which is at the same level of connection coefficients as it is the derivative of a component of frame. This scalar provides a component of the connection which does not arise in the original NP formalism, but is needed to obtain a complete set of $\Delta$-equations for the frame. From the definition of $\chi$ and the NP Ricci identities we readily obtain

$$
D \chi=2 \Phi_{11}+\Psi_{2}+\bar{\Psi}_{2}+2 \alpha \tau+2 \bar{\beta} \tau+2 \bar{\alpha} \bar{\tau}+2 \beta \bar{\tau}+2 \tau \bar{\tau}-(\epsilon+\bar{\epsilon}) \chi .
$$

Now, as a consequence of the gauge choice $Q=1$ on $\mathcal{N}_{\star}^{\prime}$, the initial data for $\chi$ on $\mathcal{N}_{\star}^{\prime}$ is 0 . For convenience we also define

$$
\varpi \equiv \beta-\bar{\alpha}
$$

corresponding to the only independent component of the connection on the spheres $\mathcal{S}_{u, v}$.

\subsubsection{The estimates}

Following the main strategy in Paper I, we construct estimates for the components of the frame and the conformal factor through the analysis of $\Delta$-equations under the following bootstrap assumption:

Assumption 2 (assumption to control the coefficients of the frame and the conformal factor) Assume that we have a solution to the vacuum conformal Einstein field equations in Stewart's gauge satisfying

$$
\left\|\left\{\chi, \mu, \lambda, \alpha, \beta, \tau, \Sigma_{2}\right\}\right\|_{L^{\infty}\left(\mathcal{S}_{u, v}\right)} \leq \Delta_{\Gamma}
$$

on a truncated causal diamond $\mathcal{D}_{u, v_{\bullet}}^{t}$, where $\Delta_{\Gamma}$ is some (possibly large) constant. 
The construction of the estimates proceeds along the following steps:

Step 1 We integrate $\chi=\Delta \log Q=\partial_{u} Q$ in the short direction so as to obtain

$$
\left|Q-Q_{\star}\right|=\left|\int_{0}^{\varepsilon} \chi \mathrm{d} u\right| \leq \int_{0}^{\varepsilon}|\chi| \mathrm{d} u \leq \int_{0}^{\varepsilon} \Delta_{\Gamma} \mathrm{d} u=\Delta_{\Gamma} \varepsilon
$$

for any $v$. Then we have

$$
\left\|Q-Q_{\star}\right\|_{L^{\infty}\left(\mathcal{S}_{u, v}\right)} \leq \Delta_{\Gamma} \varepsilon
$$

So there is a constant $C$ depending on the initial data such that

$$
Q^{-1}, \quad Q \leq C\left(\Delta_{e_{\star}}\right)
$$

Step 2 To estimate the conformal factor $\Xi$, we integrate $\Xi$ along the short direction

$$
|\Xi|=\left|\int_{0}^{\varepsilon} Q^{-1} \Sigma_{2} \mathrm{~d} u\right| \leq C\left(\Delta_{\Gamma}\right) \varepsilon
$$

Accordingly, we have the following lemma:

Lemma 6 (control of conformal factor) Under Assumption 10, if $\varepsilon>0$ is sufficiently small, there exists a constant $C$ depending on the size of the initial data such that

$$
\|\Xi\|_{L^{\infty}\left(\mathcal{S}_{u, v}\right)} \leq C\left(\Delta_{\Gamma}\right) \varepsilon
$$

on $\mathcal{D}_{u, v_{\bullet}}^{t}$.

Step 3 Integrating $P^{\mathcal{A}}$ in the short direction using Eq. (6b) one readily obtains the following lemma:

Lemma 7 (control on the components of the frame, I) Under Assumption 10, if $\varepsilon>0$ is sufficiently small, there exists a constant $C$ depending on the size of the initial data such that

$$
\left\|\left\{P^{\mathcal{A}},\left(P^{\mathcal{A}}\right)^{-1}\right\}\right\|_{L^{\infty}\left(\mathcal{S}_{u, v}\right)} \leq C\left(\Delta_{e_{\star}}\right)
$$

on $\mathcal{D}_{u, v_{\bullet}}^{t}$. Moreover, since

$$
\sigma^{\mathcal{A B}}=-P^{\mathcal{A}} \bar{P}^{\mathcal{B}}-P^{\mathcal{B}} \bar{P}^{\mathcal{A}}
$$

we also obtain that

$$
\begin{aligned}
\left|\sigma^{\mathcal{A B}}\right|,\left|\sigma_{\mathcal{A B}}\right| & \leq C\left(\Delta_{e_{\star}}\right), \\
c\left(\Delta_{e_{\star}}\right) & \leq \operatorname{det} \sigma \leq C\left(\Delta_{e_{\star}}\right) .
\end{aligned}
$$


Thus, for any vector $v^{a}$ on $\mathcal{S}_{u, v}$, we have that the norms

$$
\int_{\mathcal{S}_{u, v}}\left(\sigma_{\mathcal{A B}} v^{\mathcal{A}} v^{\mathcal{B}}\right)^{p / 2}, \quad \text { and } \quad \int_{\mathcal{S}_{u, v}}\left(\left(v^{1}\right)^{2}+\left(v^{2}\right)^{2}\right)^{p / 2},
$$

are equivalent. Finally, one also has

$$
\sup _{u, v}\left|\operatorname{Area}\left(\mathcal{S}_{u, v}\right)-\operatorname{Area}\left(\mathcal{S}_{0, v}\right)\right| \leq C \Delta_{\Gamma} \varepsilon .
$$

Step 4 Integrating $C^{\mathcal{A}}$ in the short direction using Eq. (6a) yields the lemma

Lemma 8 (control on the components of the frame, II) Choosing $\varepsilon$ suitably, since $C_{\star}^{\mathcal{A}}=0$ on $\mathscr{I}^{-}$one has that

$$
\left\|C^{\mathcal{A}}\right\|_{L^{\infty}\left(\mathcal{S}_{u, v}\right)} \leq C \Delta_{\Gamma} \varepsilon
$$

on $\mathcal{D}_{u, v}^{t}$.

\subsection{Some further tools from Paper I}

In this section we introduce some frequently used inequalities in the main estimates for the transport equations on hypersurfaces of constant $u$ or $v$. A detailed proof of these results can be found in Paper I.

\subsubsection{General estimates for transport equations}

A key tool in our analysis are formulae that allow us to follow the evolution of integrals along null hypersurfaces.

Lemma 9 (computing derivatives of integrals over $\mathcal{S}_{u, v}$ ) Given a scalar $\phi$ one has that

$$
\begin{aligned}
\frac{\mathrm{d}}{\mathrm{d} v} \int_{\mathcal{S}_{u, v}} \phi & =\int_{\mathcal{S}_{u, v}}(D \phi-2 \rho \phi), \\
\frac{\mathrm{d}}{\mathrm{d} u} \int_{\mathcal{S}_{u, v}} \phi & =\int_{\mathcal{S}_{u, v}} Q^{-1}(\Delta \phi+2 \mu \phi),
\end{aligned}
$$

along the outgoing and incoming null geodesics that rule $\mathcal{N}_{v}^{\prime}$ and $\mathcal{N}_{u}$.

Lemma 10 (integral over causal diamonds of derivatives of a scalar) Let $\phi$ be a scalar field. One has

$$
\begin{aligned}
& \int_{\mathcal{D}_{u, v}} D \phi=\int_{\mathcal{N}_{v}^{\prime}} Q^{-1} \phi-\int_{\mathcal{N}_{0}^{\prime}} Q^{-1} \phi+\int_{\mathcal{D}_{u, v}}(2 \rho+\epsilon+\bar{\epsilon}) \phi \\
& \int_{\mathcal{D}_{u, v}} \Delta \phi=\int_{\mathcal{N}_{u}} \phi-\int_{\mathcal{N}_{0}} \phi-\int_{\mathcal{D}_{u, v}} 2 \mu \phi .
\end{aligned}
$$




\subsubsection{Grönwall-type inequalities}

The following estimates will be used repeatedly in our main analysis. Again, for proofs and further discussion, the reader is referred to Paper I.

Proposition 3 (control of the $L^{p}$-norm with transport equations) We work under Assumption 10. Let $\xi$ denote a tensor field on $\mathcal{S}_{u, v}$ of arbitrary type and assume on $\mathcal{D}_{u, v_{\bullet}}^{t}$ that

$$
\sup _{u, v}\|\{\rho, \mu\}\|_{L^{\infty}\left(\mathcal{S}_{u, v}\right)} \leq \mathcal{O}
$$

Then there exists $\varepsilon_{\star}=\varepsilon_{\star}\left(\Delta_{e_{\star}}, \mathcal{O}\right)$ such that for all $\varepsilon \leq \varepsilon_{\star}$ and for every $1 \leq p<\infty$, we have the estimates

$$
\begin{aligned}
& \|\xi\|_{L^{p}\left(\mathcal{S}_{u, v}\right)} \leq C(\mathcal{O}, I)\left(\|\xi\|_{L^{p}\left(S_{u, 0}\right)}+\int_{0}^{v}\|D \xi\|_{L^{p}\left(\mathcal{S}_{u, v^{\prime}}\right)} \mathrm{d} v^{\prime}\right) \\
& \|\xi\|_{L^{p}\left(\mathcal{S}_{u, v}\right)} \leq 2\left(\|\xi\|_{L^{p}\left(\mathcal{S}_{0, v}\right)}+C\left(\Delta_{e_{\star}}, \mathcal{O}\right) \int_{0}^{u}\|\Delta \xi\|_{L^{p}\left(\mathcal{S}_{u^{\prime}, v}\right)} \mathrm{d} u^{\prime}\right)
\end{aligned}
$$

where I denotes the long direction interval.

Proposition 4 (supremum norm of solutions to transport equations) Work under Assumption 10. There exists $\varepsilon_{\star}$ such that for all $\varepsilon \leq \varepsilon_{\star}$, we have

$$
\begin{aligned}
& \|\xi\|_{L^{\infty}\left(\mathcal{S}_{u, v}\right)} \leq\|\xi\|_{L^{\infty}\left(\mathcal{S}_{u, 0}\right)}+\int_{0}^{v}\|D \xi\|_{L^{\infty}\left(\mathcal{S}_{u, v^{\prime}}\right)} \mathrm{d} v^{\prime} \\
& \|\xi\|_{L^{\infty}\left(\mathcal{S}_{u, v}\right)} \leq\|\xi\|_{L^{\infty}\left(\mathcal{S}_{0, v}\right)}+C\left(\Delta_{e_{\star}}\right) \int_{0}^{u}\|\Delta \xi\|_{L^{\infty}\left(\mathcal{S}_{u^{\prime}, v}\right)} \mathrm{d} u^{\prime} .
\end{aligned}
$$

Proposition 5 ( $L^{4}$ norm of solutions to transport equations) Work under Assumption 10 and assume on $\mathcal{D}_{u, v_{\bullet}}^{t}$ that

$$
\sup _{u, v}\|\{\rho, \mu\}\|_{L^{\infty}\left(\mathcal{S}_{u, v}\right)} \leq \mathcal{O}
$$

There exists $\varepsilon_{\star}$ such that for all $\varepsilon \leq \varepsilon_{\star}$, we have the estimates

$$
\begin{aligned}
\|\xi\|_{L^{4}\left(\mathcal{S}_{u, v}\right) \leq} & C\left(\Delta_{e_{\star}}, \mathcal{O}\right)\left(\|\xi\|_{L^{4}\left(\mathcal{S}_{u, 0}\right)}+\|D \xi\|_{L^{2}\left(\mathcal{N}_{u}(0, v)\right)}^{1 / 2}\right. \\
& \left.\left(\|\xi\|_{L^{2}\left(\mathcal{N}_{u}(0, v)\right)}^{2}+\|\not \nabla \xi\|_{L^{2}\left(\mathcal{N}_{u}(0, v)\right)}^{2}\right)^{1 / 4}\right) \\
\|\xi\|_{L^{4}\left(\mathcal{S}_{u, v}\right) \leq} & 2\left(\|\xi\|_{L^{4}\left(\mathcal{S}_{0, v}\right)}+C\left(\Delta_{e_{\star}}\right)\|\Delta \xi\|_{L^{2}\left(\mathcal{N}_{v}^{\prime}(0, u)\right)}^{1 / 2}\right. \\
& \left.\left(\|\xi\|_{L^{2}\left(\mathcal{N}_{v}^{\prime}(0, u)\right)}^{2}+\|\not \nabla \xi\|_{L^{2}\left(\mathcal{N}_{v}^{\prime}(0, u)\right)}^{2}\right)^{1 / 4}\right) .
\end{aligned}
$$




\subsubsection{Sobolev inequalities}

In this subsection we present some useful estimates of the $L^{p}$-norms of a scalar in terms of its $L^{2}$-norms and those of its derivatives. These inequalities are all derived from the isoperimetric Sobolev inequality of $\mathcal{S}_{u, v}$.

Proposition 6 (Sobolev-type inequality, I) Work under Assumption 10. Let $\phi$ be a scalar field on $\mathcal{S}_{u, v}$ which is square-integrable with square-integrable first covariant derivatives. Then for each $2<p<\infty, \phi \in L^{p}\left(\mathcal{S}_{u, v}\right)$, there exists $\varepsilon_{\star}=\varepsilon_{\star}\left(\Delta_{e_{\star}}, \Delta_{\Gamma}\right)$ such that as long as $\varepsilon \leq \varepsilon_{\star}$, we have

$$
\|\phi\|_{L^{p}\left(\mathcal{S}_{u, v}\right)} \leq G_{p}(\boldsymbol{\sigma})\left(\|\phi\|_{L^{2}\left(\mathcal{S}_{u, v}\right)}+\|\not \nabla \phi\|_{L^{2}\left(\mathcal{S}_{u, v}\right)}\right)
$$

where $G_{p}(\sigma)$ is a constant which also depends on the isoperimetric constant $\mathcal{I}\left(\mathcal{S}_{u, v}\right)$ and $p$, but is controlled by some $C\left(\Delta_{e_{\star}}\right), \nabla$ is the induced connection on $\mathcal{S}_{u, v}$ associated with the metric $\sigma$.

Moreover, we have the following:

Proposition 7 (Sobolev-type inequality, II) Work under Assumption 10. There exists $\varepsilon_{\star}=\varepsilon_{\star}\left(\Delta_{e_{\star}}, \Delta_{\Gamma}\right)$ such that as long as $\varepsilon \leq \varepsilon_{\star}$, we have

$$
\|\phi\|_{L^{\infty}\left(\mathcal{S}_{u, v}\right)} \leq G_{p}(\sigma)\left(\|\phi\|_{L^{p}\left(\mathcal{S}_{u, v}\right)}+\|\not \nabla \phi\|_{L^{p}\left(\mathcal{S}_{u, v}\right)}\right),
$$

with $2<p<\infty$ and $G_{p}(\sigma) \leq C\left(\Delta_{e_{\star}}\right)$ as above.

Corollary 2 (Sobolev-type inequality, III) Work under Assumption 10. There exists $\varepsilon_{\star}=\varepsilon_{\star}\left(\Delta_{e_{\star}}, \Delta_{\Gamma}\right)$ such that as long as $\varepsilon \leq \varepsilon_{\star}$, we have

$$
\begin{aligned}
& \|\phi\|_{L^{4}\left(\mathcal{S}_{u, v}\right)} \leq G(\boldsymbol{\sigma})\left(\|\phi\|_{L^{2}\left(\mathcal{S}_{u, v}\right)}+\|\not \nabla \phi\|_{L^{2}\left(\mathcal{S}_{u, v}\right)}\right) \\
& \|\phi\|_{L^{\infty}\left(\mathcal{S}_{u, v}\right)} \leq G(\boldsymbol{\sigma})\left(\|\phi\|_{L^{2}\left(\mathcal{S}_{u, v}\right)}+\|\not \nabla \phi\|_{L^{2}\left(\mathcal{S}_{u, v}\right)}+\left\|\not \nabla^{2} \phi\right\|_{L^{2}\left(\mathcal{S}_{u, v}\right)}\right)
\end{aligned}
$$

again with $G(\sigma) \leq C\left(\Delta_{e_{\star}}\right)$.

Remark 6 The isoperimetric constant can be shown to be controlled by the area of the surface $\mathcal{S}_{u, v}$.

\section{Main estimates}

In this section we discuss the construction of the main estimates to obtain the improved existence results for the asymptotic CIVP for the conformal Einstein field equations. The strategy of the arguments resemble that in Einstein field equations. As many of the ideas and techniques are similar to those in Paper I, as elsewhere, in this section we focus our attention on the particular aspects arising from the use of the conformal Einstein equations. 


\subsection{Norms}

The argument in this and subsequent sections relies on the use of a number of tailormade norms. We define the following:

(i) Norm for the initial value of the connection coefficients, given by

$$
\begin{aligned}
\Delta_{\Gamma_{\star}} \equiv & \sup _{\mathcal{S}_{u, v} \subset \mathscr{I}^{-}, \mathcal{N}_{\star}^{\prime} \Gamma \in\{\mu, \lambda, \rho, \sigma, \alpha, \beta, \tau, \epsilon\}} \sup \\
& \max \left\{1,\|\Gamma\|_{L^{\infty}\left(\mathcal{S}_{u, v}\right)}, \sum_{i=0}^{1}\left\|\mathbb{\nabla}^{i} \Gamma\right\|_{L^{4}\left(\mathcal{S}_{u, v}\right)}, \sum_{i=0}^{2}\left\|\nabla^{i} \Gamma\right\|_{L^{2}\left(\mathcal{S}_{u, v}\right)}\right\} .
\end{aligned}
$$

(ii) Norm for the initial value of the derivative of conformal factor $\Sigma_{a}$, given by

$$
\begin{aligned}
\Delta_{\Sigma_{\star}} \equiv & \sup _{\mathcal{S}_{u, v} \subset \mathscr{I}^{-}} \\
& \max \left\{1,\left\|\Sigma_{2}\right\|_{L^{\infty}\left(\mathcal{S}_{u, v}\right)}, \sum_{i=0}^{1}\left\|\mathbb{X}^{i} \Sigma_{2}\right\|_{L^{4}\left(\mathcal{S}_{u, v}\right)}, \sum_{i=0}^{2}\left\|\mathbb{\nabla}^{i} \Sigma_{2}\right\|_{L^{2}\left(\mathcal{S}_{u, v}\right)}\right\} .
\end{aligned}
$$

(iii) Norm for the initial value of the Ricci curvature components, given by

$$
\begin{aligned}
\Delta_{\Phi_{\star}} \equiv & \sup _{\mathcal{S}_{u, v} \subset \mathscr{I}^{-}, \mathcal{N}_{\star}^{\prime} \Phi \in\left\{\Phi_{00}, \Phi_{01}, \Phi_{02}, \Phi_{11}, \Phi_{12}\right\}} \sup \\
& \max \left\{1, \sum_{i=0}^{1}\left\|\mathbb{X}^{i} \Phi\right\|_{L^{4}\left(\mathcal{S}_{u, v}\right)}, \sum_{i=0}^{2}\left\|\mathbb{X}^{i} \Phi\right\|_{L^{2}\left(\mathcal{S}_{u, v}\right)}\right\} \\
& +\sum_{i=0}^{3} \sup _{\Phi \in\left\{\Phi_{00}, \Phi_{01}, \Phi_{02}, \Phi_{11}, \Phi_{12}\right\}}\left\|\mathbb{X}^{i} \Phi\right\|_{L^{2}(\mathscr{I}-)} \\
& +\sup _{\Phi \in\left\{\Phi_{01}, \Phi_{02}, \Phi_{11}, \Phi_{12}, \Phi_{22}\right\}}\left\|\mathbb{X}^{i} \Phi\right\|_{L^{2}\left(\mathcal{N}_{\star}^{\prime}\right)} .
\end{aligned}
$$

(iv) Norm for the initial value of the rescaled Weyl curvature components, given by

$$
\begin{aligned}
\Delta_{\phi_{\star}} \equiv & \sup _{\mathcal{S}_{u, v} \subset \mathscr{I}-, \mathcal{N}_{\star}^{\prime} \phi \in\left\{\phi_{0}, \phi_{1}, \phi_{2}, \phi_{3}, \phi_{4}\right\}} \sup \\
& \max \left\{1, \sum_{i=0}^{1}\left\|\mathbb{\nabla}^{i} \phi\right\|_{L^{4}\left(\mathcal{S}_{u, v}\right)}, \sum_{i=0}^{2}\left\|\mathbb{X}^{i} \phi\right\|_{L^{2}\left(\mathcal{S}_{u, v}\right)}\right\} \\
& +\sum_{i=0}^{3} \sup _{\phi \in\left\{\phi_{0}, \phi_{1}, \phi_{2}, \phi_{3}\right\}}\left\|\mathbb{\nabla}^{i} \phi\right\|_{L^{2}\left(\mathscr{I}^{-}\right)}+\sup _{\phi \in\left\{\phi_{1}, \phi_{2}, \phi_{3}, \phi_{4}\right\}}\left\|\mathbb{P}^{i} \phi\right\|_{L^{2}\left(\mathcal{N}_{\star}^{\prime}\right)} .
\end{aligned}
$$


(v) Norm for the components of the Ricci curvature components at later null hypersurfaces, given by

$$
\begin{aligned}
\Delta_{\Phi} \equiv & \sum_{i=0}^{3} \sup _{\Phi \in\left\{\Phi_{00}, \Phi_{01}, \Phi_{02}, \Phi_{11}, \Phi_{12}\right\}}\left\|\mathbb{\nabla}^{i} \Phi\right\|_{L^{2}\left(\mathcal{N}_{u}^{t}\right)} \\
& +\sup _{\Phi \in\left\{\Phi_{01}, \Phi_{02}, \Phi_{11}, \Phi_{12}, \Phi_{22}\right\}}\left\|\mathbb{\nabla}^{i} \Phi\right\|_{L^{2}\left(\mathcal{N}_{v}^{\prime t}\right)},
\end{aligned}
$$

where the suprema in $u$ and $v$ are taken over $\mathcal{D}_{u, v_{\bullet}}^{t}$.

(vi) Supremum-type norm over the $L^{2}$-norm of the components of the Ricci curvature at spheres of constant $u, v$, given by

$$
\Delta_{\Phi}(\mathcal{S}) \equiv \sum_{i=0}^{2} \sup _{u, v}\left\|\mathbb{\nabla}^{i}\left\{\Phi_{00}, \Phi_{01}, \Phi_{02}, \Phi_{11}, \Phi_{12}\right\}\right\|_{L^{2}\left(\mathcal{S}_{u, v}\right)},
$$

where the supremum is taken over $\mathcal{D}_{u, v_{\bullet}}^{t}$.

(vii) Norm for the components of the Weyl tensor at later null hypersurfaces, given by

$$
\Delta_{\phi} \equiv \sum_{i=0}^{3} \sup _{\phi \in\left\{\phi_{0}, \phi_{1}, \phi_{2}, \phi_{3}\right\}}\left\|\mathbb{\nabla}^{i} \phi\right\|_{L^{2}\left(\mathcal{N}_{u}^{t}\right)}+\sup _{\phi \in\left\{\phi_{1}, \phi_{2}, \phi_{3}, \phi_{4}\right\}}\left\|\mathbb{Z}^{i} \phi\right\|_{L^{2}\left(\mathcal{N}_{v}^{\prime t}\right)},
$$

where the suprema in $u$ and $v$ are taken over $\mathcal{D}_{u, v_{\bullet}}^{t}$.

(viii) Supremum-type norm over the $L^{2}$-norm of the components of the rescaled Weyl curvature at spheres of constant $u, v$, given by,

$$
\Delta_{\phi}(\mathcal{S}) \equiv \sum_{i=0}^{2} \sup _{u, v}\left\|\mathbb{X}^{i}\left\{\phi_{0}, \phi_{1}, \phi_{2}, \phi_{3}\right\}\right\|_{L^{2}\left(\mathcal{S}_{u, v}\right)},
$$

with the supremum taken over $\mathcal{D}_{u, v_{\bullet}}^{t}$ and in which $u$ will be taken sufficiently small to apply our estimates.

\subsection{Estimates for the connection coefficients and the derivative of conformal factor}

In this subsection, we prove estimates for connection coefficients and derivatives of the conformal factor. We assume first that the norms of curvature are bounded and prove that the short range $\varepsilon$ can be chosen such that connection coefficients and the derivative of conformal factor can be controlled by initial data and $\Delta_{\Phi}(\mathcal{S})$. This can be achieved by considering the transport equations. For the connection coefficients $\tau$ and $\chi$, we only have their long direction $D$ equations. However, the fact that there is no quadratic term in $\tau$ or $\chi$ themselves allows us to regard these as linear equations for $\tau$ 
and $\chi$. Then the Grönwall-type inequalities will show us that these two connection coefficients are bounded. Accordingly, except for $\tau$ and $\chi$, we can analyse the $\Delta$ equations for the connection coefficients and the derivatives of conformal. The small range of $\varepsilon$ does not let them drift too far from their initial data on $\mathscr{I}^{-}$. Consequently, we find that although $\Sigma_{1}, \Sigma_{3}$ and $\Sigma_{4}$ are all small, the component $\Sigma_{1}$ has a different power of $\varepsilon$ than $\Sigma_{3}$ and $\Sigma_{4}$ in our estimates.

Proposition 8 (control on the supremum norm of the connection coefficients and the derivatives of the conformal factor) Assume that we have a solution of the vacuum conformal Einstein field equations in Stewart's gauge in a region $\mathcal{D}_{u, v_{\bullet}}^{t}$ with

$$
\sup _{u, v}\left\|\left\{\mu, \lambda, \alpha, \beta, \epsilon, \rho, \sigma, \tau, \chi, \Sigma_{1}, \Sigma_{2}, \Sigma_{3}, \Sigma_{4}\right\}\right\|_{L^{\infty}\left(\mathcal{S}_{u, v}\right)} \leq \Delta_{\Gamma, \Sigma}
$$

for some positive $\Delta_{\Gamma, \Sigma}$. Assume also that

$$
\begin{aligned}
& \Delta_{\Phi}(\mathcal{S})<\infty, \quad \Delta_{\Phi}<\infty, \quad \Delta_{\phi}(\mathcal{S})<\infty, \quad \Delta_{\phi}<\infty, \\
& \sup _{u, v}\left\|\mathbb{X}^{i} \tau\right\|_{L^{2}\left(\mathcal{S}_{u, v}\right)}<\infty, \quad i=2,3,
\end{aligned}
$$

on the same domain. Then there exists

$$
\varepsilon_{\star}=\varepsilon_{\star}\left(I, \Delta_{e_{\star}}, \Delta_{\Gamma_{\star}}, \sup _{u, v}\left\|\mathbb{D}^{2} \tau\right\|_{L^{2}\left(\mathcal{S}_{u, v}\right)}, \sup _{u, v}\left\|\not \mathbb{P}^{3} \tau\right\|_{L^{2}\left(\mathcal{S}_{u, v}\right)}, \Delta_{\Sigma_{\star}}, \Delta_{\phi}, \Delta_{\Phi}\right),
$$

such that when $\varepsilon \leq \varepsilon_{\star}$, we have

$$
\begin{aligned}
& \sup _{u, v}\|\{\mu, \lambda, \rho, \sigma, \alpha, \beta, \epsilon\}\|_{L^{\infty}\left(\mathcal{S}_{u, v}\right)} \leq 3 \Delta_{\Gamma_{\star}}, \\
& \sup _{u, v}\|\{\tau, \chi\}\|_{L^{\infty}\left(\mathcal{S}_{u, v}\right) \leq C\left(I, \Delta_{e_{\star}}, \Delta_{\Gamma_{\star}}, \Delta_{\Phi}(\mathcal{S})\right),} \sup _{u, v}\left\|\Sigma_{2}\right\|_{L^{\infty}\left(\mathcal{S}_{u, v}\right) \leq 3 \Delta_{\Sigma_{\star}},} \\
& \sup _{u, v}\left\|\left\{\Sigma_{1}, \Sigma_{3}, \Sigma_{4}\right\}\right\|_{L^{\infty}\left(\mathcal{S}_{u, v}\right)} \leq C\left(I, \Delta_{e_{\star}}, \Delta_{\Gamma_{\star}}, \Delta_{\Sigma_{\star}}, \Delta_{\Phi}(\mathcal{S})\right) \varepsilon, \\
& \sup _{u, v}\|s\|_{L^{\infty}\left(\mathcal{S}_{u, v}\right)} \leq C\left(\Delta_{e_{\star}}, \Delta_{\Sigma_{\star}}, \Delta_{\Phi}\right) \varepsilon^{1 / 2},
\end{aligned}
$$

on $\mathcal{D}_{u, v_{\bullet}}^{t}$.

Proof Basic bootstrap assumption Place the following bootstrap assumptions:

$$
\begin{aligned}
& \sup _{u, v}\|\{\mu, \lambda, \rho, \sigma, \alpha, \beta, \epsilon\}\|_{L^{\infty}\left(\mathcal{S}_{u, v}\right)} \leq 4 \Delta_{\Gamma_{\star}}, \\
& \sup _{u, v}\left\|\left\{\Sigma_{1}, \Sigma_{2}, \Sigma_{3}, \Sigma_{4}\right\}\right\|_{L^{\infty}\left(\mathcal{S}_{u, v}\right)} \leq 4 \Delta_{\Sigma_{\star}} .
\end{aligned}
$$


Estimate for $\tau$. First we prove that $\|\tau\|_{L^{\infty}\left(\mathcal{S}_{u, v}\right)} \leq C\left(I, \Delta_{e_{\star}}, \Delta_{\Gamma_{\star}}, \Delta_{\Phi}(\mathcal{S})\right)$. We make use of the $D$-direction equation of $\tau,(13 \mathrm{~b})$,

$$
D \tau=(\epsilon-\bar{\epsilon}+\rho) \tau+\sigma \bar{\tau}+\bar{\pi} \rho+\pi \sigma+\Xi \phi_{1}+\Phi_{01} .
$$

The above equation crucially contains no $\tau^{2}$ terms. Making use of the Sobolev inequality in the Proposition 7, we obtain that

$$
\begin{aligned}
\left\|\phi_{i}\right\|_{L^{\infty}\left(\mathcal{S}_{u, v}\right)} & \leq \Delta_{\phi}(\mathcal{S})<\infty, \quad i=0,1,2,3 \\
\left\|\Phi_{H}\right\|_{L^{\infty}\left(\mathcal{S}_{u, v}\right)} & \leq \Delta_{\Phi}(\mathcal{S})<\infty
\end{aligned}
$$

where $\Phi_{H}=\left\{\Phi_{00}, \Phi_{01}, \Phi_{02}, \Phi_{11}, \Phi_{12}\right\}$. Then the inequalities in Proposition 4 show that

$$
\begin{aligned}
\|\tau\|_{L^{\infty}\left(\mathcal{S}_{u, v}\right) \leq} & \|\tau\|_{L^{\infty}\left(\mathcal{S}_{u, 0}\right)}+\int_{0}^{v}\|D \tau\|_{L^{\infty}\left(\mathcal{S}_{u, v^{\prime}}\right)} \mathrm{d} v^{\prime} \\
\leq & \Delta_{\Gamma_{\star}}+C\left(\Delta_{\Gamma_{\star}}, \Delta_{e_{\star}}, \Delta_{\Phi}(\mathcal{S})\right) v_{\bullet}+C\left(I, \Delta_{\Sigma_{\star}}, \Delta_{e_{\star}}, \Delta_{\phi}(\mathcal{S})\right) \varepsilon \\
& +C\left(\Delta_{\Gamma_{\star}}\right) \int_{0}^{v}\|\tau\|_{L^{\infty}\left(\mathcal{S}_{u, v^{\prime}}\right)} \mathrm{d} v^{\prime} .
\end{aligned}
$$

Now, choosing $\varepsilon$ sufficiently small, it follows from Grönwall's inequality that

$$
\|\tau\|_{L^{\infty}\left(\mathcal{S}_{u, v}\right)} \leq C\left(I, \Delta_{e_{\star}}, \Delta_{\Gamma_{\star}}, \Delta_{\Phi}(\mathcal{S})\right)
$$

Estimate for $\chi$. In order to estimate $\chi$, we use the $D$-direction Eq. (9) for $\chi$. A similar analysis as before yields

$$
\|\chi\|_{L^{\infty}\left(\mathcal{S}_{u, v}\right)} \leq C\left(I, \Delta_{e_{\star}}, \Delta_{\Gamma_{\star}}, \Delta_{\Phi}(\mathcal{S})\right)
$$

Estimates for $\mu, \lambda, \alpha, \beta$ and $\epsilon$. To estimate the coefficients $\mu$ and $\lambda$, we consider Eq. (13g) and (13o):

$$
\begin{aligned}
\Delta \mu & =-\mu^{2}-\lambda \bar{\lambda}-\Phi_{22}, \\
\Delta \lambda & =-2 \mu \lambda-\Xi \phi_{4} .
\end{aligned}
$$

Making use of the inequalities in Proposition 4 for the short direction, we obtain that

$$
\begin{aligned}
\|\mu\|_{L^{\infty}\left(\mathcal{S}_{u, v}\right)} & \leq\|\mu\|_{L^{\infty}\left(\mathcal{S}_{0, v}\right)}+C\left(\Delta_{e_{\star}}\right) \int_{0}^{\varepsilon}\|\Delta \mu\|_{L^{\infty}\left(\mathcal{S}_{u^{\prime}, v}\right)} \mathrm{d} u^{\prime} \\
& \leq \Delta_{\Gamma_{\star}}+C\left(\Delta_{e_{\star}}, \Delta_{\Gamma_{\star}}\right) \varepsilon+C\left(\Delta_{e_{\star}}\right) \int_{0}^{u}\left\|\Phi_{22}\right\|_{L^{\infty}\left(\mathcal{S}_{u^{\prime}, v}\right)} \mathrm{d} u^{\prime} .
\end{aligned}
$$


From the Sobolev and Hölder inequalities, we further find that

$$
\begin{aligned}
\int_{0}^{u}\left\|\Phi_{22}\right\|_{L^{\infty}\left(\mathcal{S}_{u^{\prime}, v}\right)} \mathrm{d} u^{\prime} & \leq C\left(\Delta_{e_{\star}}\right) \int_{0}^{u} \sum_{i=0}^{2}\left\|\nabla^{i} \Phi_{22}\right\|_{L^{2}\left(\mathcal{S}_{u^{\prime}, v}\right)} \mathrm{d} u^{\prime} \\
& =\sum_{i=0}^{2} C\left(\Delta_{e_{\star}}\right) \int_{0}^{u}\left(\int_{\mathcal{S}}\left|\mathbb{\nabla}^{i} \Phi_{22}\right|^{2}\right)^{1 / 2} \mathrm{~d} u^{\prime} \\
& \leq\left(\sum_{i=0}^{2} C\left(\Delta_{e_{\star}}\right) \int_{0}^{u} \int_{\mathcal{S}}\left|\mathbb{X}^{i} \Phi_{22}\right|^{2} \mathrm{~d} u^{\prime}\right)^{1 / 2}\left(\int_{0}^{u} 1 \mathrm{~d} u^{\prime}\right)^{1 / 2} \\
& \leq C\left(\Delta_{e_{\star}}\right) \varepsilon^{1 / 2}|| \mathbb{X}^{i} \Phi_{22} \|_{L^{2}\left(\mathcal{N}_{v}^{\prime}(0, u)\right)}
\end{aligned}
$$

Hence we obtain that

$$
\|\mu\|_{L^{\infty}\left(\mathcal{S}_{u, v}\right)} \leq \Delta_{\Gamma_{\star}}+C\left(\Delta_{e_{\star}}, \Delta_{\Gamma_{\star}}\right) \varepsilon+C \varepsilon^{1 / 2} \Delta_{\Phi} .
$$

For the connection coefficient $\lambda$, a similar computation yields

$$
\begin{aligned}
\|\lambda\|_{L^{\infty}\left(\mathcal{S}_{u, v}\right)} & \leq \Delta_{\Gamma_{\star}}+C\left(\Delta_{e_{\star}}, \Delta_{\Gamma_{\star}}\right) \varepsilon+C\left(\Delta_{e_{\star}}\right) \int_{0}^{u}\left\|\Xi \phi_{4}\right\|_{L^{\infty}\left(\mathcal{S}_{u^{\prime}, v}\right)} d u^{\prime}, \\
& \leq \Delta_{\Gamma_{\star}}+C\left(\Delta_{e_{\star}}, \Delta_{\Gamma_{\star}}\right) \varepsilon+C \varepsilon^{3 / 2} \Delta_{\phi} .
\end{aligned}
$$

With the same method, we can estimate $\alpha, \beta$ and $\epsilon$ by using their short direction structure Eqs. (13k), (13d) and (13a):

$$
\begin{aligned}
\Delta \alpha & =-\mu \alpha-\lambda \beta-\lambda \tau-\Xi \phi_{3}, \\
\Delta \beta & =-\bar{\lambda} \alpha-\mu \beta-\tau \mu-\Phi_{12}, \\
\Delta \epsilon & =-\alpha \bar{\pi}-\beta \pi-\alpha \tau-\beta \bar{\tau}-\pi \tau-\Xi \phi_{2}-\Phi_{11} .
\end{aligned}
$$

The details are omitted.

Estimates for $\rho$ and $\sigma$. In this case, the relevant $\Delta$-transport equations are the structure Eqs. (13i) and (13r):

$$
\begin{aligned}
\Delta \rho & =\bar{\delta} \tau-\mu \rho-\lambda \sigma-\alpha \tau+\bar{\beta} \tau-\tau \bar{\tau}-\Xi \phi_{2}, \\
\Delta \sigma & =\delta \tau-\bar{\lambda} \rho-\mu \sigma+\bar{\alpha} \tau-\beta \tau-\tau^{2}-\Phi_{02} .
\end{aligned}
$$

In order to estimate $\delta \tau$ and $\bar{\delta} \tau$, we make use of the Sobolev inequalities in Corollary 2 and partial integration on $\mathcal{S}_{u, v}$ to obtain

$$
\begin{aligned}
\|\not \nabla \tau\|_{L^{\infty}\left(\mathcal{S}_{u, v}\right)} \leq & C\left(\Delta_{e_{\star}}\right) \sum_{i=1}^{3}\left\|\nabla^{i} \tau\right\|_{L^{2}\left(\mathcal{S}_{u, v}\right)} \leq C\left(\Delta_{e_{\star}}\right)\left(\|\tau\|_{L^{2}\left(\mathcal{S}_{u, v}\right)}\right. \\
& \left.+\left\|\mathbb{D}^{2} \tau\right\|_{L^{2}\left(\mathcal{S}_{u, v}\right)}+\left\|\mathbb{D}^{3} \tau\right\|_{L^{2}\left(\mathcal{S}_{u, v}\right)}\right)
\end{aligned}
$$


Then the Hölder inequality

$$
\|\tau\|_{L^{2}\left(\mathcal{S}_{u, v}\right)} \leq\|\tau\|_{L^{\infty}\left(\mathcal{S}_{u, v}\right)} \operatorname{Area}(\mathcal{S})^{1 / 2}
$$

and the assumptions

$$
\sup _{u, v}\left\|\mathbb{D}^{2} \tau\right\|_{L^{2}\left(\mathcal{S}_{u, v}\right)}, \quad \sup _{u, v}\left\|\mathbb{X}^{3} \tau\right\|_{L^{2}\left(\mathcal{S}_{u, v}\right)}<\infty
$$

show us that $\|\mathbb{\nabla} \tau\|_{L^{\infty}\left(\mathcal{S}_{u, v}\right)}$ is bounded. So we can estimate the $\|\mathbb{\nabla} \tau\|_{L^{\infty}\left(\mathcal{S}_{u, v}\right)}$ term in the short direction using Eqs. (13i) and (13r) for $\sigma$ and $\rho$, respectively.

Estimate for $s$. Before estimating the derivatives of the conformal factor, we first analyse the Friedrich scalar $s$. Making use of the conformal Einstein field Eq. (17b),

$$
\Delta s=-\Sigma_{1} \Phi_{22}-\Sigma_{2} \Phi_{11}+\Sigma_{3} \Phi_{21}+\Sigma_{4} \Phi_{12}
$$

and the initial value $s{\mid \mathscr{I}_{-}}^{=} 0$, we readily have that

$$
\begin{aligned}
\|s\|_{L^{\infty}\left(\mathcal{S}_{u, v}\right)} & \leq C\left(\Delta_{e_{\star}}\right) \int_{0}^{u}\left\|\Sigma_{2} \Phi_{11}-\Sigma_{4} \Phi_{12}-\Sigma_{3} \Phi_{21}+\Sigma_{1} \Phi_{22}\right\|_{L^{\infty}\left(\mathcal{S}_{u^{\prime}, v}\right)} \mathrm{d} u^{\prime} \\
& \leq C\left(\Delta_{e_{\star}}, \Delta_{\Sigma_{\star}}, \Delta_{\Phi}(\mathcal{S})\right) \varepsilon+C\left(\Delta_{e_{\star}}, \Delta_{\Sigma_{\star}}, \Delta_{\Phi}\right) \varepsilon^{1 / 2} .
\end{aligned}
$$

Estimate for $\Sigma_{2}$. Making use of the conformal Einstein field Eq. (15e)

$$
\Delta \Sigma_{2}=-\Xi \Phi_{22}
$$

we have that

$$
\begin{aligned}
\left\|\Sigma_{2}\right\|_{L^{\infty}\left(\mathcal{S}_{u, v}\right)} & \leq \Delta_{\Sigma_{\star}}+C\left(\Delta_{e_{\star}}\right) \int_{0}^{u}\left\|\Xi \Phi_{22}\right\|_{L^{\infty}\left(\mathcal{S}_{u^{\prime}, v}\right.} \mathrm{d} u^{\prime} \\
& \leq \Delta_{\Sigma_{\star}}+C\left(\Delta_{e_{\star}}, \Delta_{\Sigma_{\star}}, \Delta_{\Phi}\right) \varepsilon^{3 / 2} .
\end{aligned}
$$

Thus, we can choose $\varepsilon_{\star}$ sufficiently small such that $\left\|\Sigma_{2}\right\|_{L^{\infty}\left(\mathcal{S}_{u, v}\right)}$ remains close to its initial value.

Estimate for $\Sigma_{1}$. Next, Eq. (15d)

$$
\Delta \Sigma_{1}=-\Sigma_{4} \tau-\Sigma_{3} \bar{\tau}+s-\Xi \Phi_{11}
$$

and the initial value $\left.\Sigma_{1}\right|_{\mathscr{I}^{-}}=0$, gives that

$$
\begin{aligned}
\left\|\Sigma_{1}\right\|_{L^{\infty}\left(\mathcal{S}_{u, v}\right) \leq} & C\left(\Delta_{e_{\star}}\right) \int_{0}^{u}\left\|-\Sigma_{4} \tau-\Sigma_{3} \bar{\tau}+s-\Xi \Phi_{11}\right\|_{L^{\infty}\left(S_{u^{\prime}, v}\right)} \mathrm{d} u^{\prime} \\
\leq & C\left(I, \Delta_{e_{\star}}, \Delta_{\Gamma_{\star}}, \Delta_{\Sigma_{\star}}, \Delta_{\Phi}(\mathcal{S})\right) \varepsilon+C\left(\Delta_{e_{\star}}, \Delta_{\Sigma_{\star}}, \Delta_{\Phi}\right) \varepsilon^{3 / 2} \\
& +C\left(I, \Delta_{e_{\star}}, \Delta_{\Gamma_{\star}}, \Delta_{\Sigma_{\star}}, \Delta_{\Phi}(\mathcal{S})\right) \varepsilon^{2} .
\end{aligned}
$$


Estimates for $\Sigma_{3}$ and $\Sigma_{4}$. Equation (15f)

$$
\Delta \Sigma_{3}=-\Sigma_{2} \tau-\Xi \Phi_{12}
$$

readily gives that

$$
\begin{aligned}
\left\|\Sigma_{3}\right\|_{L^{\infty}\left(\mathcal{S}_{u, v}\right)} & \left.\leq C\left(\Delta_{e_{\star}}\right) \int_{0}^{u}\left\|\Sigma_{2} \tau+\Xi \Phi_{12}\right\|_{L^{\infty}\left(S_{u^{\prime}, v}\right.}\right) \mathrm{d} u^{\prime}, \\
& \leq C\left(I, \Delta_{e_{\star}}, \Delta_{\Gamma_{\star}}, \Delta_{\Sigma_{\star}}, \Delta_{\Phi}(\mathcal{S})\right) \varepsilon+C\left(\Delta_{e_{\star}}, \Delta_{\Sigma_{\star}}, \Delta_{\Phi}(\mathcal{S})\right) \varepsilon^{2} .
\end{aligned}
$$

The method is the same for $\Sigma_{4}$.

Concluding the argument. From the estimates for the NP connection coefficients and $\Sigma_{A A^{\prime}}$ constructed above it follows that one can choose

$$
\begin{aligned}
\varepsilon_{\star} & =\varepsilon_{\star}\left(I, \Delta_{e_{\star}}, \Delta_{\Gamma_{\star}}, \sup _{u, v}\left\|\mathbb{D}^{2} \tau\right\|_{L^{2}\left(\mathcal{S}_{u, v}\right)},\right. \\
& \left.\sup _{u, v}\left\|\mathbb{D}^{3} \tau\right\|_{L^{2}\left(\mathcal{S}_{u, v}\right)}, \Delta_{\Sigma_{\star}}, \Delta_{\phi}, \Delta_{\Phi}, \Delta_{\phi}, \Delta_{\Phi}(\mathcal{S})\right),
\end{aligned}
$$

sufficiently small so that

$$
\begin{aligned}
& \sup _{u, v}\|\{\mu, \lambda, \alpha, \beta, \epsilon, \rho, \sigma\}\|_{L^{\infty}\left(\mathcal{S}_{u, v}\right)} \leq 3 \Delta_{\Gamma_{\star}}, \\
& \sup _{u, v}\left\|\Sigma_{2}\right\|_{L^{\infty}\left(\mathcal{S}_{u, v}\right) \leq 3 \Delta_{\Sigma_{\star}},} \\
& \sup _{u, v}\left\|\left\{\Sigma_{1}, \Sigma_{3}, \Sigma_{4}\right\}\right\|_{L^{\infty}\left(\mathcal{S}_{u, v}\right)} \leq C\left(I, \Delta_{e_{\star}}, \Delta_{\Gamma_{\star}}, \Delta_{\Sigma_{\star}}, \Delta_{\Phi}(\mathcal{S})\right) \varepsilon .
\end{aligned}
$$

Accordingly, we have improved our initial bootstrap assumption.

Now we use the similar method to analyse the $L^{4}$ estimate of the connection coefficients and the derivative of conformal factor.

Proposition 9 (control on the $L^{4}$-norm of the connection coefficients and the derivatives of the conformal factor) With the same assumptions in Proposition 8, and additionally assuming that

$$
\sup _{u, v}\left\|\mathbb{Z}\left\{\mu, \lambda, \alpha, \beta, \epsilon, \rho, \sigma, \Sigma_{1}, \Sigma_{2}, \Sigma_{3}, \Sigma_{4}\right\}\right\|_{L^{4}\left(\mathcal{S}_{u, v}\right)} \leq \Delta_{\Gamma, \Sigma},
$$

in the truncated diamond $\mathcal{D}_{u, v_{\bullet}}^{t}$, we find that there exists

$$
\begin{gathered}
\varepsilon_{\star}=\varepsilon_{\star}\left(I, \Delta_{e_{\star}}, \Delta_{\Gamma_{\star}}, \sup _{u, v}\left\|\not \mathbb{D}^{2} \tau\right\|_{L^{2}\left(\mathcal{S}_{u, v}\right)}, \sup _{u, v}\left\|\mathbb{D}^{3} \tau\right\|_{L^{2}\left(\mathcal{S}_{u, v}\right)},\right. \\
\left.\Delta_{\Sigma_{\star}}, \Delta_{\phi}, \Delta_{\Phi}, \Delta_{\phi}(\mathcal{S}), \Delta_{\Phi}(\mathcal{S})\right),
\end{gathered}
$$


such that when $\varepsilon \leq \varepsilon_{\star}$, we have

$$
\begin{aligned}
& \sup _{u, v}\|\mathbb{X}\{\tau, \chi\}\|_{L^{4}\left(\mathcal{S}_{u, v}\right)} \leq C\left(I, \Delta_{e_{\star}}, \Delta_{\Gamma_{\star}}, \Delta_{\Phi}(\mathcal{S})\right), \\
& \sup _{u, v}\|\mathbb{X}\{\mu, \lambda, \rho, \sigma, \alpha, \beta, \epsilon\}\|_{L^{4}\left(\mathcal{S}_{u, v}\right)} \leq 3 \Delta_{\Gamma_{\star}}, \\
& \sup _{u, v}\left\|\mathbb{D} \Sigma_{2}\right\|_{L^{4}\left(\mathcal{S}_{u, v}\right)} \leq 3 \Delta_{\Sigma_{\star}}, \\
& \sup _{u, v}\left\|\mathbb{D}\left\{\Sigma_{1}, \Sigma_{3}, \Sigma_{4}\right\}\right\|_{L^{4}\left(\mathcal{S}_{u, v}\right)} \leq C\left(I, \Delta_{e_{\star}}, \Delta_{\Gamma_{\star}}, \Delta_{\Sigma_{\star}}, \Delta_{\Phi}(\mathcal{S})\right) \varepsilon .
\end{aligned}
$$

on $\mathcal{D}_{u, v_{\bullet}}^{t}$.

Proof Basic bootstrap assumption We make bootstrap assumptions

$$
\begin{aligned}
& \sup _{u, v}\|\mathbb{Z}(\mu, \lambda, \rho, \sigma, \alpha, \beta, \epsilon)\|_{L^{4}\left(\mathcal{S}_{u, v}\right)} \leq 4 \Delta_{\Gamma_{\star}} \\
& \sup _{u, v}\left\|\mathbb{Z}\left\{\Sigma_{1}, \Sigma_{2}, \Sigma_{3}, \Sigma_{4}\right\}\right\|_{L^{4}\left(\mathcal{S}_{u, v}\right)} \leq 4 \Delta_{\Sigma_{\star}} .
\end{aligned}
$$

Estimates for $\not \nabla \tau$. First, we estimate the $L^{4}\left(\mathcal{S}_{u, v}\right)$ norm of $\not \nabla \tau$. Apply the $\delta$-derivative to the $D$-direction equation of $\tau$ and the commutator of directional covariant derivatives we obtain

$$
\begin{aligned}
D \delta \tau= & (\rho+\bar{\rho}+2 \epsilon-2 \bar{\epsilon}) \delta \tau+\sigma \bar{\delta} \tau+\sigma \delta \bar{\tau}+\delta(\epsilon-\bar{\epsilon}+\rho) \tau+\bar{\tau} \delta \sigma+\rho \delta \bar{\pi} \\
& +\bar{\pi} \delta \rho+\sigma \delta \pi+\pi \delta \sigma+\Gamma^{3}+\Sigma_{3} \phi_{1}+\Xi \delta \phi_{1}+\Xi \phi_{1} \Gamma+\delta \Phi_{01}+\Phi_{01} \Gamma .
\end{aligned}
$$

In order to estimate the terms in $\|\Gamma \not \Gamma \Gamma\|_{L^{4}\left(\mathcal{S}_{u, v}\right)}$, we use the Hölder inequality and split it as

$$
\|\Gamma \not \nabla \Gamma\|_{L^{4}\left(\mathcal{S}_{u, v}\right)} \leq\|\Gamma\|_{L^{\infty}\left(\mathcal{S}_{u, v}\right)}\|\not \nabla \Gamma\|_{L^{4}\left(\mathcal{S}_{u, v}\right)}
$$

Now, Proposition 8 shows that terms of the form $\|\Gamma\|_{L^{\infty}\left(\mathcal{S}_{u, v}\right)}$ are, in fact, bounded. Making use of the Sobolev inequality in Proposition 6 and the long direction inequality in Proposition 5, we find that

$$
\begin{aligned}
\| \delta \tau & \left\|_{L^{4}\left(\mathcal{S}_{u, v}\right)}+\right\| \bar{\delta} \tau \|_{L^{4}\left(\mathcal{S}_{u, v}\right)} \\
\leq & C\left(\|\delta \tau\|_{L^{4}\left(S_{u, 0}\right)}+\|\bar{\delta} \tau\|_{L^{4}\left(S_{u, 0}\right)}+\int_{0}^{v}\|D \delta \tau\|_{L^{4}\left(S_{u, v^{\prime}}\right)}+\|D \bar{\delta} \tau\|_{L^{4}\left(S_{u, v^{\prime}}\right)} \mathrm{d} v^{\prime}\right) \\
\leq & C\left(I, \Delta_{e_{\star}}, \Delta_{\Gamma_{\star}}, \Delta_{\Phi}(\mathcal{S})\right)+C\left(I, \Delta_{e_{\star}}, \Delta_{\Gamma_{\star}}, \Delta_{\Sigma_{\star}}, \Delta_{\Phi}(\mathcal{S}), \Delta_{\phi}(\mathcal{S})\right) \varepsilon \\
& +C\left(\Delta_{\Gamma_{\star}}\right) \int_{0}^{v}\left(\|\delta \tau\|_{L^{4}\left(\mathcal{S}_{u, v^{\prime}}\right)}+\|\bar{\delta} \tau\|_{L^{4}\left(\mathcal{S}_{u, v^{\prime}}\right)}\right) \mathrm{d} v^{\prime} .
\end{aligned}
$$


Thus Grönwall's inequality gives

$$
\begin{aligned}
\|\delta \tau\|_{L^{4}\left(\mathcal{S}_{u, v}\right)}+\|\bar{\delta} \tau\|_{L^{4}\left(\mathcal{S}_{u, v}\right) \leq} & C\left(I, \Delta_{e_{\star}}, \Delta_{\Gamma_{\star}}, \Delta_{\Phi}(\mathcal{S})\right) \\
& +C\left(I, \Delta_{e_{\star}}, \Delta_{\Gamma_{\star}}, \Delta_{\Sigma_{\star}}, \Delta_{\Phi}(\mathcal{S}), \Delta_{\phi}(\mathcal{S})\right) \varepsilon .
\end{aligned}
$$

Accordingly, for a small range $\varepsilon$, we obtain that

$$
\|\mathbb{\nabla} \tau\|_{L^{4}\left(\mathcal{S}_{u, v}\right)} \leq C\left(I, \Delta_{e_{\star}}, \Delta_{\Gamma_{\star}}, \Delta_{\Phi}(\mathcal{S})\right) .
$$

Estimates for $\not \chi$. A direct computation shows that

$$
\begin{aligned}
D \delta \chi & =(\bar{\rho}-2 \bar{\epsilon}) \delta \chi+\sigma \bar{\delta} \chi+\Gamma \delta \Gamma-\chi \delta(\epsilon+\bar{\epsilon})+\Sigma_{3}\left(\phi_{2}+\bar{\phi}_{2}\right) \\
& +\Xi \delta\left(\phi_{2}+\bar{\phi}_{2}\right)+\delta \Phi_{11},
\end{aligned}
$$

where $\Gamma$ represents a combination of the connection coefficients whose particular form is not required. A similar equation can be obtained for $D \bar{\delta} \chi$. Using the same method as for the coefficient $\tau$, we obtain that $\|\mathbb{\nabla} \chi\|_{L^{4}\left(\mathcal{S}_{u, v}\right)} \leq C\left(I, \Delta_{e_{\star}}, \Delta_{\Gamma_{\star}}, \Delta_{\Phi}(\mathcal{S})\right)$.

Estimates for $\not\{\mu, \lambda, \rho, \sigma, \alpha, \beta, \epsilon\}$. Applying the operator $\Delta$ to Eqs. (13g) and (13o) we find that

$$
\begin{aligned}
\Delta \delta \mu & =(\tau-\bar{\alpha}-\beta)\left(\mu^{2}+\lambda \bar{\lambda}\right)-3 \mu \delta \mu-\bar{\lambda} \bar{\delta} \mu-\lambda \delta \bar{\lambda}-\bar{\lambda} \delta \lambda-\delta \Phi_{22}, \\
\Delta \delta \lambda & =(\tau-\bar{\alpha}-\beta)\left(2 \mu \lambda+\Xi \phi_{4}\right)-3 \mu \delta \lambda-\bar{\lambda} \bar{\delta} \lambda-2 \lambda \delta \mu-\Sigma_{3} \phi_{4}-\Xi \delta \phi_{4} .
\end{aligned}
$$

Now, a direct computation applying Proposition 3 shows that we can find an $\varepsilon_{\star}$ such that when $\varepsilon \leq \varepsilon_{\star}$, we have

$$
\|\mathbb{X}\{\mu, \lambda\}\|_{L^{4}\left(\mathcal{S}_{u, v}\right)} \leq 3 \Delta_{\Gamma_{\star}}
$$

We can estimate $\delta \alpha, \delta \beta$ and $\delta \epsilon$ by using the same method. Since we are using the assumption $\sup _{u, v}\left\|\nabla^{3} \tau\right\|_{L^{2}\left(\mathcal{S}_{u, v}\right)}<\infty$ in the truncated causal diamond, the Sobolev inequalities of Corollary 2 show that $\left\|\not \mathbb{Z}^{2} \tau\right\|_{L^{4}\left(\mathcal{S}_{u, v}\right)}$ is finite. Proceeding in a similar way we can estimate $\delta \sigma$ and $\delta \rho$ by applying $\delta$ to Eqs. (13i) and (13r).

Estimate for $\not \nabla \Sigma_{2}$. Applying $\delta$ to the short direction Eq. (15e) for $\Sigma_{2}$ and using the commutators we find that

$$
\begin{aligned}
\Delta \delta \Sigma_{2}= & -\Xi \delta \Phi_{22}-\Sigma_{3} \Phi_{22}+\Xi \Phi_{22}(\tau-\bar{\pi})+\Xi \Phi_{21} \bar{\lambda}+\Xi \Phi_{12} \mu, \\
& +\Sigma_{2}(\pi \bar{\lambda}+\bar{\pi} \mu)-\Sigma_{3}\left(\lambda \bar{\lambda}+\mu^{2}\right)-2 \Sigma_{4} \bar{\lambda} \bar{\mu} .
\end{aligned}
$$

Similar arguments to the ones used for the connection coefficients show that

$$
\begin{aligned}
\left\|\mathbb{} \Sigma_{2}\right\|_{L^{4}\left(\mathcal{S}_{u, v}\right) \leq} & 2 \Delta_{\Sigma_{\star}}+C\left(I, \Delta_{e_{\star}}, \Delta_{\Gamma_{\star}}, \Delta_{\Sigma_{\star}}\right) \varepsilon \\
& +C\left(I, \Delta_{e_{\star}}, \Delta_{\Gamma_{\star}}, \Delta_{\Sigma_{\star}}, \Delta_{\Phi}(\mathcal{S})\right) \varepsilon^{2}+o\left(\varepsilon^{2}\right) .
\end{aligned}
$$


Accordingly, the $\varepsilon_{\star}$ can be chosen sufficiently small to ensure that $\left\|\not \nabla \Sigma_{2}\right\|_{L^{4}\left(\mathcal{S}_{u, v}\right)}$ is no more than $3 \Delta_{\Sigma_{\star}}$.

Estimate for $\not \nabla \Sigma_{1}$. Making use of the equation for $\Delta \delta \Sigma_{1}$ :

$$
\begin{aligned}
\Delta \delta \Sigma_{1}= & -\Sigma_{1} \Phi_{12}-\Sigma_{2} \Phi_{01}+\Sigma_{4} \Phi_{02}+s(\bar{\pi}-\tau)+\Xi \Phi_{11}(\tau-\bar{\pi})+\Sigma_{4} \tau(\tau-\bar{\pi}) \\
& +\Sigma_{3} \bar{\tau}(\tau-\bar{\pi})-\mu \delta \Sigma_{1}-\bar{\tau} \delta \Sigma_{3}-\tau \delta \Sigma_{4}-\Xi \delta \Phi_{11}-\Sigma_{4} \delta \tau-\Sigma_{3} \delta \bar{\tau}-\bar{\lambda} \bar{\delta} \Sigma_{1},
\end{aligned}
$$

it follows from the bootstrap assumption, that

$$
\left\|\mathbb{Z} \Sigma_{1}\right\|_{L^{4}\left(\mathcal{S}_{u, v}\right)} \leq C\left(I, \Delta_{e_{\star}}, \Delta_{\Gamma_{\star}}, \Delta_{\Sigma_{\star}}, \Delta_{\Phi}(\mathcal{S})\right) \varepsilon+o(\varepsilon) .
$$

Estimate for $\not \nabla \Sigma_{3,4}$. A direct computation yields the equation

$$
\begin{aligned}
\Delta \delta \Sigma_{3}= & -\Sigma_{3} \Phi_{12}+\Xi \Phi_{12}(\tau-\bar{\pi})+\Sigma_{2} \tau(\tau-\bar{\pi})-\tau \delta \Sigma_{2} \\
& -\Sigma_{2} \delta \tau-\mu \delta \Sigma_{3}-\Xi \delta \Phi_{12}-\bar{\lambda} \delta \Sigma_{3} .
\end{aligned}
$$

accordingly, one can readily find that

$$
\left\|\mathbb{Z} \Sigma_{3}\right\|_{L^{4}\left(\mathcal{S}_{u, v}\right)} \leq C\left(I, \Delta_{e_{\star}}, \Delta_{\Gamma_{\star}}, \Delta_{\Sigma_{\star}}, \Delta_{\Phi}(\mathcal{S})\right) \varepsilon+o(\varepsilon) .
$$

A similar result holds for $\left\|\mathbb{Z} \Sigma_{4}\right\|_{L^{4}(S)}$. It follows from the previous discussion that when $\varepsilon$ is suitably small, we can improve the bootstrap assumption.

Concluding the argument. From the analysis above, it follows we can choose

$$
\begin{aligned}
& \varepsilon_{\star}= \varepsilon_{\star}\left(I, \Delta_{e_{\star}}, \Delta_{\Gamma_{\star}}, \sup _{u, v}\left\|\mathbb{X}^{2} \tau\right\|_{L^{2}\left(\mathcal{S}_{u, v}\right)}, \sup _{u, v}\left\|\mathbb{Z}^{3} \tau\right\|_{L^{2}\left(\mathcal{S}_{u, v}\right)}, \Delta_{\Sigma_{\star}},\right. \\
&\left.\Delta_{\phi}, \Delta_{\Phi}, \Delta_{\phi}(\mathcal{S}), \Delta_{\Phi}(\mathcal{S})\right),
\end{aligned}
$$

sufficiently small so that

$$
\begin{aligned}
& \sup _{u, v}\|\mathbb{Z}\{\mu, \lambda, \rho, \sigma, \alpha, \beta, \epsilon\}\|_{L^{4}\left(\mathcal{S}_{u, v}\right)} \leq 3 \Delta_{\Gamma_{\star}}, \\
& \sup _{u, v}\left\|\mathbb{Z} \Sigma_{2}\right\|_{L^{4}\left(\mathcal{S}_{u, v}\right)} \leq 3 \Delta_{\Sigma_{\star}}, \\
& \sup _{u, v}\left\|\mathbb{X}\left\{\Sigma_{1}, \Sigma_{3}, \Sigma_{4}\right\}\right\|_{L^{4}\left(\mathcal{S}_{u, v}\right)} \leq C\left(I, \Delta_{e_{\star}}, \Delta_{\Gamma_{\star}}, \Delta_{\Sigma_{\star}}, \Delta_{\Phi}(\mathcal{S})\right) \varepsilon .
\end{aligned}
$$

The above estimates improve the bootstrap assumption.

The discussion of this section is concluded with $L^{2}$-estimates for the connection coefficients and the derivative of conformal factor.

Proposition 10 (control on the $L^{2}$-norm of the connection coefficients and the derivatives of the conformal factor) Assume that we have a solution of the vacuum conformal Einstein field equations in Stewart's gauge in a region $\mathcal{D}_{u, v}^{t}$. with 


$$
\begin{aligned}
& \sup _{u, v}\left\|\left\{\mu, \lambda, \alpha, \beta, \epsilon, \rho, \sigma, \tau, \chi, \Sigma_{1}, \Sigma_{2}, \Sigma_{3}, \Sigma_{4}\right\}\right\|_{L^{\infty}\left(S_{u, v}\right)} \leq \Delta_{\Gamma, \Sigma}, \\
& \sup _{u, v}\left\|\mathbb{X}\left\{\mu, \lambda, \alpha, \beta, \epsilon, \rho, \sigma, \Sigma_{1}, \Sigma_{2}, \Sigma_{3}, \Sigma_{4}\right\}\right\|_{L^{4}\left(\mathcal{S}_{u, v}\right)} \leq \Delta_{\Gamma, \Sigma}, \\
& \sup _{u, v}\left\|\mathbb{X}^{2}\left\{\mu, \lambda, \alpha, \beta, \epsilon, \rho, \sigma, \tau, \Sigma_{1}, \Sigma_{2}, \Sigma_{3}, \Sigma_{4}\right\}\right\|_{L^{2}\left(\mathcal{S}_{u, v}\right)} \leq \Delta_{\Gamma, \Sigma},
\end{aligned}
$$

for some positive $\Delta_{\Gamma, \Sigma}$. Assume also

$$
\sup _{u, v}\left\|\mathbb{X}^{3} \tau\right\|_{L^{2}\left(S_{u, v}\right)}<\infty, \quad \Delta_{\Phi}(\mathcal{S})<\infty, \quad \Delta_{\Phi}<\infty, \quad \Delta_{\phi}(\mathcal{S})<\infty, \quad \Delta_{\phi}<\infty
$$

on the same domain. We have that there exists

$$
\varepsilon_{\star}=\varepsilon_{\star}\left(I, \Delta_{e_{\star}}, \Delta_{\Gamma_{\star}}, \Delta_{\Sigma_{\star}} \sup _{u, v}\left\|\nabla^{3} \tau\right\|_{L^{2}\left(\mathcal{S}_{u, v}\right)}, \Delta_{\phi}, \Delta_{\Phi}, \Delta_{\phi}(\mathcal{S}), \Delta_{\Phi}(\mathcal{S})\right),
$$

such that when $\varepsilon \leq \varepsilon_{\star}$, we have that

$$
\begin{aligned}
& \sup _{u, v}\left\|\mathbb{D}^{2}\{\tau, \chi\}\right\|_{L^{2}\left(\mathcal{S}_{u, v}\right)} \leq C\left(I, \Delta_{e_{\star}}, \Delta_{\Gamma_{\star}}, \Delta_{\Phi}(\mathcal{S})\right), \\
& \sup _{u, v}\left\|\mathbb{D}^{2}\{\mu, \lambda, \alpha, \beta, \epsilon, \rho, \sigma\}\right\|_{L^{2}\left(\mathcal{S}_{u, v}\right)} \leq 3 \Delta_{\Gamma_{\star}}, \\
& \sup _{u, v}\left\|\mathbb{D}^{2} \Sigma_{2}\right\|_{L^{4}\left(\mathcal{S}_{u, v}\right)} \leq 3 \Delta_{\Sigma_{\star}}, \\
& \sup _{u, v}\left\|\mathbb{D}^{2}\left\{\Sigma_{1}, \Sigma_{3}, \Sigma_{4}\right\}\right\|_{L^{4}\left(\mathcal{S}_{u, v}\right)} \leq C\left(I, \Delta_{e_{\star}}, \Delta_{\Gamma_{\star}}, \Delta_{\Sigma_{\star}}, \Delta_{\Phi}(\mathcal{S})\right) \varepsilon .
\end{aligned}
$$

Proof Basic bootstrap assumption. We make following bootstrap assumptions:

$$
\begin{aligned}
& \sup _{u, v}\left\|\mathbb{Z}^{2}\{\mu, \lambda, \rho, \sigma, \alpha, \beta, \epsilon\}\right\|_{L^{2}\left(\mathcal{S}_{u, v}\right)} \leq 4 \Delta_{\Gamma_{\star}}, \\
& \sup _{u, v}\left\|\mathbb{D}^{2}\left\{\Sigma_{1}, \Sigma_{2}, \Sigma_{3}, \Sigma_{4}\right\}\right\|_{L^{2}\left(\mathcal{S}_{u, v}\right)} \leq 4 \Delta_{\Sigma_{\star}} .
\end{aligned}
$$

Estimates for $\left\|\nabla^{2} \tau\right\|_{L^{2}\left(\mathcal{S}_{u, v}\right)}$ and $\left\|\nabla^{2} \chi\right\|_{L^{2}\left(\mathcal{S}_{u, v}\right)}$. Applying the operator $\delta$ to the equation for $D \delta \tau$ and using the commutators, one obtains following the $D$-direction equation of $\delta^{2} \tau$ :

$$
\begin{aligned}
D \delta^{2} \tau= & \Gamma \delta^{2} \tau+\Gamma \delta^{2} \bar{\tau}+\Gamma \bar{\delta} \delta \tau+\Gamma \delta \bar{\delta} \tau+\Gamma_{1}^{4}+\Gamma_{1} \delta^{2} \Gamma_{1} \\
& +\delta \Gamma_{1} \delta \Gamma_{1}+\Gamma_{1}^{2} \delta \Gamma_{1}+\delta^{2} \Phi_{01}+\Gamma_{1} \delta \Phi_{01}+\Phi_{01} \delta \Gamma_{1}+\Phi_{01} \Gamma_{1}^{2} \\
& +\delta \Sigma_{3} \phi_{1}+2 \Sigma_{3} \delta \phi_{1}+\Xi \delta \phi_{1}+\Xi \phi_{1} \Gamma^{2}+\Xi \Gamma_{1} \delta \phi_{1}+\Xi \phi_{1} \delta \Gamma_{1}+\Xi \delta^{2} \phi_{1} \\
& +\Sigma_{3} \phi_{1} \Gamma_{1}
\end{aligned}
$$


where $\Gamma$ contains a combination of the coefficients $\rho, \sigma, \epsilon, \Gamma_{1}$ contains a combination of $\tau, \alpha, \beta, \sigma, \epsilon, \rho$. A similar computation renders equations for $D \bar{\delta} \tau, D \delta \bar{\delta} \tau$. Terms of the form $\delta \Gamma_{1} \delta \Gamma_{1}$ can be handled using the Hölder inequality

$$
\left\|\delta \Gamma_{1} \delta \Gamma_{1}\right\|_{L^{2}\left(\mathcal{S}_{u, v}\right)} \leq\left\|\delta \Gamma_{1}\right\|_{L^{4}\left(\mathcal{S}_{u, v}\right)}|| \delta \Gamma_{1} \|_{L^{4}\left(\mathcal{S}_{u, v}\right)},
$$

where Proposition 9 shows that the bound is finite. The analysis for the term $\delta \Sigma_{3} \phi_{1}$ is the same. More precisely, one has that

$$
\begin{aligned}
\left\|\delta \Sigma_{3} \phi_{1}\right\|_{L^{2}\left(\mathcal{S}_{u, v}\right)} & \leq\left\|\delta \Sigma_{3}\right\|_{L^{4}\left(\mathcal{S}_{u, v}\right)}\left\|\phi_{1}\right\|_{L^{4}\left(\mathcal{S}_{u, v}\right)} \\
& \leq C\left(\Delta_{e_{\star}}\right)\left\|\delta \Sigma_{3}\right\|_{L^{4}\left(\mathcal{S}_{u, v}\right)}\left(\left\|\phi_{1}\right\|_{L^{2}\left(\mathcal{S}_{u, v}\right)}+\left\|\not \nabla \phi_{1}\right\|_{L^{2}\left(\mathcal{S}_{u, v}\right)}\right) .
\end{aligned}
$$

Similar arguments can be employed in the rest of the terms for the equation for $D \delta^{2} \tau$ so that with the long direction inequality in Proposition 3 we obtain

$$
\begin{aligned}
\left\|\delta^{2} \tau\right\|_{L^{2}\left(\mathcal{S}_{u, v}\right)} \leq & C\left(I, \Delta_{\Gamma_{\star}}\right)\left(\left\|\delta^{2} \tau\right\|_{L^{2}\left(\mathcal{S}_{u, 0}\right)}+\int_{0}^{v}\left\|D \delta^{2} \tau\right\|_{L^{2}\left(\mathcal{S}_{u, v^{\prime}}\right)} \mathrm{d} v^{\prime}\right), \\
\leq & C\left(I, \Delta_{e_{\star}}, \Delta_{\Gamma_{\star}}, \Delta_{\Phi}(\mathcal{S})\right)+C\left(I, \Delta_{e_{\star}}, \Delta_{\Gamma_{\star}}, \Delta_{\Sigma_{\star}}, \Delta_{\Phi}(\mathcal{S}), \Delta_{\phi}(\mathcal{S})\right) \varepsilon \\
& +C\left(I, \Delta_{e_{\star}}, \Delta_{\Gamma_{\star}}\right) \int_{0}^{v}\left\|\nabla^{2} \tau\right\|_{L^{2}\left(\mathcal{S}_{u, v^{\prime}}\right)} \mathrm{d} v^{\prime} .
\end{aligned}
$$

Similar estimates can be obtained for $\bar{\delta}^{2} \tau, \delta \bar{\delta} \tau$ and $\bar{\delta} \delta \tau$. To estimate $\|\delta \tau\|_{L^{2}\left(\mathcal{S}_{u, v}\right)}$ we can make use of the fact that the area of $\mathcal{S}_{u, v}$ is bounded so that

$$
\|\delta \tau\|_{L^{2}\left(\mathcal{S}_{u, v}\right)} \leq C\left(\Delta_{e_{\star}}, \Delta_{\Gamma_{\star}}\right)\|\delta \tau\|_{L^{4}\left(\mathcal{S}_{u, v}\right)},
$$

hence, Proposition 9 shows us that this is also finite. From inequality (32) of Paper I we get

$$
\begin{aligned}
\left\|\mathbb{X}^{2} \tau\right\|_{L^{2}\left(\mathcal{S}_{u, v}\right) \leq} & C\left(I, \Delta_{e_{\star}}, \Delta_{\Gamma_{\star}}, \Delta_{\Phi}(\mathcal{S})\right)+C\left(I, \Delta_{e_{\star}}, \Delta_{\Gamma_{\star}}, \Delta_{\Gamma_{\star}}, \Delta_{\Phi}(\mathcal{S}), \Delta_{\phi}(\mathcal{S})\right) \varepsilon \\
& +C\left(I, \Delta_{e_{\star}}, \Delta_{\Gamma_{\star}}\right) \int_{0}^{v}\left\|\mathbb{Z}^{2} \tau\right\|_{L^{2}\left(\mathcal{S}_{u, v^{\prime}}\right)} \mathrm{d} v^{\prime},
\end{aligned}
$$

so that using Grönwall's inequality we conclude that

$$
\left\|\mathbb{Z}^{2} \tau\right\|_{L^{2}\left(\mathcal{S}_{u, v}\right)} \leq C\left(I, \Delta_{e_{\star}}, \Delta_{\Gamma_{\star}}, \Delta_{\Phi}(\mathcal{S})\right)+C\left(I, \Delta_{e_{\star}}, \Delta_{\Gamma_{\star}}, \Delta_{\phi}(\mathcal{S})\right) \varepsilon .
$$

Hence, one finds that $\left\|\not \nabla^{2} \tau\right\|_{L^{2}\left(\mathcal{S}_{u, v}\right)}$ is bounded by a constant $C\left(I, \Delta_{e_{\star}}, \Delta_{\Gamma_{\star}}, \Delta_{\Phi}(\mathcal{S})\right)$. Using the same analysis, we can conclude that $\left\|\nabla^{2} \chi\right\|_{L^{2}\left(\mathcal{S}_{u, v}\right)}$ is bounded.

Estimates for the remaining spin connection coefficients. Estimates for the remaining connection coefficients can be obtained by the same methods as in Proposition 9 namely, first we compute equations for $\Delta \delta^{2} \Gamma$ and $\Delta \bar{\delta} \delta \Gamma$, and make use of the short direction inequality in Proposition 3 to find that 


$$
\left\|\mathbb{X}^{2}\{\mu, \lambda, \alpha, \beta, \epsilon, \sigma, \rho\}\right\|_{L^{2}\left(\mathcal{S}_{u, v}\right)} \leq 3 \Delta_{\Gamma_{\star}}
$$

for sufficiently small $\varepsilon$.

Estimates for $\nabla^{2} \Sigma_{2}$. A direct calculation shows that

$$
\begin{aligned}
\Delta \delta^{2} \Sigma_{2}= & \Gamma \delta^{2} \Sigma_{2}+\delta \Gamma \delta \Sigma_{2}+\Gamma^{2} \delta \Sigma_{2}+\Xi \delta^{2} \Phi_{22}+\Xi \Phi_{22} \delta \Gamma \\
& +\Phi_{22} \delta \Sigma_{3}+\Sigma_{3} \delta \Phi_{22}+\Xi \Gamma \delta \Phi_{22}+\Sigma_{3} \Phi_{22} \Gamma+\Xi \Phi_{22} \Gamma^{2} .
\end{aligned}
$$

The other short direction equation for the remaining second order spherical derivatives of $\Sigma_{2}$ have the same structure. From these equations we obtain that

$$
\left\|\nabla^{2} \Sigma_{2}\right\|_{L^{2}\left(\mathcal{S}_{u, v}\right)} \leq 2 \Delta_{\Sigma_{\star}}+C\left(I, \Delta_{e_{\star}}, \Delta_{\Gamma_{\star}}, \Delta_{\Sigma_{\star}}, \Delta_{\Phi}(\mathcal{S})\right) \varepsilon+o(\varepsilon) .
$$

The term $o(\varepsilon)$ arises from the presence of $\delta^{i} \Xi, i=0,1,2$.

Estimates for $\not^{2} \Sigma_{1}$ and $\not^{2} \Sigma_{3,4}$. Again, a direct computation yields the equation

$$
\begin{aligned}
\Delta \delta^{2} \Sigma_{1}= & \Gamma \delta^{2} \Sigma_{1}+\Gamma \delta^{2} \Sigma^{\prime}+\Sigma \Gamma \delta \Gamma+\delta \Sigma \delta \Gamma+\Sigma \delta^{2} \Gamma+\Sigma \Gamma^{3}+\Gamma^{2} \delta \Sigma+\Gamma \Sigma \Phi, \\
& +\Gamma^{2} \Xi \Phi+s \Gamma^{2}+s \delta \Gamma+\Phi \delta \Sigma+\Sigma \delta \Phi+\Xi \Gamma \delta \Phi+\Xi \Phi \delta \Gamma+\Xi \delta^{2} \Phi,
\end{aligned}
$$

where $\Gamma$ contains $\tau, \Sigma$ contains $\Sigma_{2}$, and $\Sigma^{\prime}$ does not contain $\Sigma_{1}$, while $\Phi$ does not contain $\Phi_{22}$. Making use of the same arguments as for $\Sigma_{2}$, we obtain that

$$
\left\|\mathbb{W}^{2} \Sigma_{1}\right\|_{L^{2}\left(\mathcal{S}_{u, v}\right)} \leq C\left(I, \Delta_{e_{\star}}, \Delta_{\Gamma_{\star}}, \Delta_{\Sigma_{\star}}, \Delta_{\Phi}(\mathcal{S})\right) \varepsilon+o(\varepsilon)
$$

Similar arguments give

$$
\left\|\mathbb{W}^{2} \Sigma_{3}\right\|_{L^{2}\left(\mathcal{S}_{u, v}\right)} \leq C\left(I, \Delta_{e_{\star}}, \Delta_{\Gamma_{\star}}, \Delta_{\Sigma_{\star}}, \Delta_{\Phi}(\mathcal{S})\right) \varepsilon+o(\varepsilon)
$$

Concluding the argument. From the analysis in the previous paragraphs it follows that we can choose

$$
\varepsilon_{\star}=\varepsilon_{\star}\left(I, \Delta_{e_{\star}}, \Delta_{\Gamma_{\star}}, \sup _{u, v}\left\|\mathbb{D}^{3} \tau\right\|_{L^{2}\left(\mathcal{S}_{u, v}\right)}, \Delta_{\Sigma_{\star}}, \Delta_{\phi}, \Delta_{\Phi}, \Delta_{\phi}(\mathcal{S}), \Delta_{\Phi}(\mathcal{S})\right),
$$

sufficiently small so that

$$
\begin{aligned}
& \sup _{u, v}\left\|\mathbb{D}^{2}\{\mu, \lambda, \rho, \sigma, \alpha, \beta, \epsilon\}\right\|_{L^{2}\left(\mathcal{S}_{u, v}\right)} \leq 3 \Delta_{\Gamma_{\star}}, \\
& \sup _{u, v}\left\|\mathbb{X}^{2} \Sigma_{2}\right\|_{L^{2}\left(\mathcal{S}_{u, v}\right)} \leq 3 \Delta_{\Sigma_{\star}}, \\
& \sup _{u, v}\left\|\mathbb{D}^{2}\left\{\Sigma_{1}, \Sigma_{3}, \Sigma_{4}\right\}\right\|_{L^{2}\left(\mathcal{S}_{u, v}\right)} \leq C\left(I, \Delta_{e_{\star}}, \Delta_{\Gamma_{\star}}, \Delta_{\Sigma_{\star}}, \Delta_{\Phi}(\mathcal{S})\right) \varepsilon .
\end{aligned}
$$

The above estimates improve the bootstrap assumptions. 


\subsection{First estimates for the curvature}

Building on the $L^{p}$-estimates for the connection coefficients and the derivative of the conformal factor obtained in the previous section, we now show that the norms $\Delta_{\Phi}(\mathcal{S})$ and $\Delta_{\phi}(\mathcal{S})$ are bounded by the initial data. This is achieved in the next two propositions.

Proposition 11 (basic control of the Ricci curvature) Assume that we are given a solution to the vacuum CEFEs in Stewart's gauge satisfying the assumptions of Proposition 10. Then there exists

$$
\varepsilon_{\star}=\varepsilon_{\star}\left(I, \Delta_{e_{\star}}, \Delta_{\Gamma_{\star}}, \Delta_{\Sigma_{\star}}, \Delta_{\Phi_{\star}}, \Delta_{\Phi}, \Delta_{\phi}, \sup _{u, v}\left\|\mathbb{Z}^{3} \tau\right\|_{L^{2}\left(\mathcal{S}_{u, v}\right)}\right)
$$

such that for $\varepsilon \leq \varepsilon_{\star}$, we have

$$
\Delta_{\Phi}(\mathcal{S})<3 \Delta_{\Phi_{\star}}
$$

on $\mathcal{D}_{u, v_{\bullet}}^{t}$.

Proof Bootstrap assumption. We make the following bootstrap assumption:

$$
\sup _{u, v}\left\|\mathbb{D}^{i}\left\{\Phi_{00}, \Phi_{01}, \Phi_{02}, \Phi_{11}, \Phi_{12}\right\}\right\|_{L^{2}\left(\mathcal{S}_{u, v}\right)} \leq 4 \Delta_{\Phi_{\star}}, \quad i=0, \ldots, 2 .
$$

$L^{2}$-norm of the components $\left\{\Phi_{00}, \Phi_{01}, \Phi_{02}, \Phi_{11}, \Phi_{12}\right\}$. We focus on the $L^{2}(\mathcal{S})$ norm of $\left\{\Phi_{00}, \Phi_{01}, \Phi_{02}, \Phi_{11}, \Phi_{12}\right\}$. We will use the short direction Eqs. (19a)-(19e) to estimate these components. We take $\Phi_{11}$ as an example. The relevant equation is in this case given by

$$
\Delta \Phi_{11}=\delta \Phi_{21}+2 \beta \Phi_{21}-\bar{\lambda} \Phi_{20}-2 \mu \Phi_{11}+\bar{\rho} \Phi_{22}-\tau \Phi_{21}-\bar{\tau} \Phi_{21}+\Sigma_{2} \bar{\phi}_{2}-\Sigma_{4} \bar{\phi}_{3} .
$$

It follows then that

$$
\begin{aligned}
\| & \Phi_{11} \|_{L^{2}\left(\mathcal{S}_{u, v}\right)} \\
\leq & 2\left(\left\|\Phi_{11}\right\|_{L^{2}\left(\mathcal{S}_{0, v}\right)}+C\left(\Delta_{e_{\star}}, \Delta_{\Gamma_{\star}}\right) \int_{0}^{u}\left\|\Delta \Phi_{11}\right\|_{L^{2}\left(\mathcal{S}_{u^{\prime}, v}\right)} \mathrm{d} u^{\prime}\right), \\
\leq & 2 \Delta_{\Phi_{\star}}+C\left(\Delta_{e_{\star}}, \Delta_{\Gamma_{\star}}\right) \int_{0}^{u}\left(\left\|\delta \Phi_{21}\right\|_{L^{2}\left(\mathcal{S}_{u^{\prime}, v}\right)}+\left\|\bar{\rho} \Phi_{22}\right\|_{L^{2}\left(\mathcal{S}_{u^{\prime}, v}\right)}+\left\|\Sigma_{2} \bar{\phi}_{2}\right\|_{L^{2}\left(\mathcal{S}_{u^{\prime}, v}\right)},\right. \\
& \left.+\left\|2 \beta \Phi_{21}+\bar{\lambda} \Phi_{20}+2 \mu \Phi_{11}+\tau \Phi_{21}+\bar{\tau} \Phi_{21}\right\|_{L^{2}\left(\mathcal{S}_{u^{\prime}, v}\right)}+\left\|\Sigma_{4} \bar{\phi}_{3}\right\|_{L^{2}\left(\mathcal{S}_{u^{\prime}, v}\right)}\right) \mathrm{d} u^{\prime} .
\end{aligned}
$$

Using the Hölder inequality, the first three terms can be transformed to a norm on the light cone. More precisely, one has 


$$
\begin{aligned}
\int_{0}^{u}\left\|\delta \Phi_{21}\right\|_{L^{2}\left(\mathcal{S}_{u^{\prime}, v}\right)} d u^{\prime} & =\int_{0}^{u}\left(\int_{\mathcal{S}_{u^{\prime}, v}}\left|\delta \Phi_{21}\right|^{2}\right)^{1 / 2} \mathrm{~d} u^{\prime} \\
& \leq\left(\int_{0}^{u} \int_{\mathcal{S}_{u^{\prime}, v}}\left|\delta \Phi_{21}\right|^{2}\right)^{1 / 2}\left(\int_{0}^{u} 1\right)^{1 / 2} \\
& \leq \varepsilon^{1 / 2}|| \delta \Phi_{21} \|_{L^{2}\left(\mathcal{N}_{v}^{\prime}(0, u)\right)} \leq \Delta_{\Phi} \varepsilon^{1 / 2}
\end{aligned}
$$

Similarly, one has that

$$
\begin{aligned}
\int_{0}^{u} & \left\|\bar{\rho} \Phi_{22}\right\|_{L^{2}\left(\mathcal{S}_{u^{\prime}, v}\right)} d u^{\prime} \leq C\left(\Delta_{\Gamma_{\star}}, \Delta_{\Phi}\right) \varepsilon^{1 / 2} \\
& \int_{0}^{u}\left\|\Sigma_{2} \bar{\phi}_{2}\right\|_{L^{2}\left(\mathcal{S}_{u^{\prime}, v}\right)} d u^{\prime} \leq C\left(\Delta_{\Sigma_{\star}}, \Delta_{\phi}\right) \varepsilon^{1 / 2} .
\end{aligned}
$$

The (large) fourth term can be estimated as follows:

$$
\int_{0}^{u}\|\Gamma \Phi\|_{L^{2}\left(\mathcal{S}_{u^{\prime}, v}\right)} \mathrm{d} u^{\prime} \leq \int_{0}^{u}\|\Gamma\|_{L^{\infty}\left(\mathcal{S}_{u^{\prime}, v}\right)}\|\Phi\|_{L^{2}\left(\mathcal{S}_{u^{\prime}, v}\right)} \mathrm{d} u^{\prime} \leq C\left(I, \Delta_{e_{\star}}, \Delta_{\Gamma_{\star}}, \Delta_{\Phi_{\star}}\right) \varepsilon .
$$

For the last term we have that

$$
\begin{aligned}
\int_{0}^{u}\left\|\Sigma_{4} \bar{\phi}_{3}\right\|_{L^{2}\left(\mathcal{S}_{u^{\prime}, v}\right)} d u^{\prime} & \leq \int_{0}^{u}\left\|\Sigma_{4}\right\|_{L^{\infty}\left(\mathcal{S}_{u^{\prime}, v}\right)}\left\|\bar{\phi}_{3}\right\|_{L^{2}\left(\mathcal{S}_{u^{\prime}, v}\right)} \mathrm{d} u^{\prime} \\
& \leq C \varepsilon\left\|\phi_{3}\right\|_{L^{2}\left(\mathcal{N}_{v}^{\prime}(0, u)\right)} \varepsilon^{1 / 2} \leq C\left(I, \Delta_{e_{\star}}, \Delta_{\Gamma_{\star}}, \Delta_{\Sigma_{\star}}, \Delta_{\phi}\right) \varepsilon^{3 / 2}
\end{aligned}
$$

Hence, we find that

$$
\begin{aligned}
\left\|\Phi_{11}\right\|_{L^{2}\left(\mathcal{S}_{u, v}\right) \leq} & 2 \Delta_{\Phi_{\star}}+C\left(\Delta_{e_{\star}}, \Delta_{\Sigma_{\star}}, \Delta_{\Gamma_{\star}}, \Delta_{\Phi}, \Delta_{\phi}\right) \varepsilon^{1 / 2}+C\left(I, \Delta_{e_{\star}}, \Delta_{\Gamma_{\star}}, \Delta_{\Phi_{\star}}\right) \varepsilon \\
& +C\left(I, \Delta_{e_{\star}}, \Delta_{\Gamma_{\star}}, \Delta_{\Sigma_{\star}}, \Delta_{\phi}\right) \varepsilon^{3 / 2}
\end{aligned}
$$

Accordingly, $\varepsilon_{\star}$ can be chosen sufficiently small so that $\left\|\Phi_{11}\right\|_{L^{2}\left(\mathcal{S}_{u, v}\right)}$ is less than $3 \Delta_{\Phi_{\star}}$, and similarly for the remaining terms. Consequently, we have improved the bootstrap assumption and finished Step 1, that is, we have

$$
\sup _{u, v}\left\|\left(\Phi_{00}, \Phi_{01}, \Phi_{02}, \Phi_{11}, \Phi_{12}\right)\right\|_{L^{2}\left(\mathcal{S}_{u, v}\right)} \leq 3 \Delta_{\Phi_{\star}}
$$

Estimates for $\left\|\not \mathbb{Z}\left\{\Phi_{00}, \Phi_{01}, \Phi_{02}, \Phi_{11}, \Phi_{12}\right\}\right\|_{L^{2}\left(\mathcal{S}_{u, v}\right)}$. We now focus on the $L^{2}\left(\mathcal{S}_{u, v}\right)$ norm of the first derivative of the Ricci curvature. We take $\not \nabla \Phi_{11}$ as an example. Using the results of Proposition 3 we readily have 


$$
\begin{aligned}
& \left\|\mathbb{P} \Phi_{11}\right\|_{L^{2}\left(\mathcal{S}_{u, v}\right)} \\
& \quad \leq 2\left(\left\|\not \nabla \Phi_{11}\right\|_{L^{2}\left(\mathcal{S}_{0, v}\right)}+C\left(\Delta_{e_{\star}}, \Delta_{\Gamma_{\star}}\right) \int_{0}^{u}\left(\int_{\mathcal{S}_{u^{\prime}, v}} \Delta\left\langle\not \nabla \Phi_{11}, \not \nabla \Phi_{11}\right\rangle_{\sigma}\right)^{1 / 2} \mathrm{~d} u^{\prime}\right), \\
& \leq 2 \Delta_{\Phi_{\star}}+C\left(\Delta_{e_{\star}}, \Delta_{\Gamma_{\star}}\right) \int_{0}^{u}\left(\int_{\mathcal{S}_{u^{\prime}, v}}\left|\not \nabla \Phi_{11}\right|\left(\left|\Delta \delta \Phi_{11}\right|+\left|\Delta \bar{\delta} \Phi_{11}\right|\right)\right)^{1 / 2} \mathrm{~d} u^{\prime},
\end{aligned}
$$

while the short direction equation for $\delta \Phi_{11}$ is given by

$$
\begin{aligned}
\Delta \delta \Phi_{11}= & \delta^{2} \Phi_{21}+\Sigma_{2} \bar{\phi}_{2}(\bar{\pi}-\tau)+\bar{\phi}_{2} \delta \Sigma_{2}+\Sigma_{2} \delta \bar{\phi}_{2} \\
& +\Sigma_{4} \bar{\phi}_{3}(\tau-\bar{\pi})-\bar{\phi}_{3} \delta \Sigma_{4}+\Sigma_{4} \delta \bar{\phi}_{3} \\
& +\Phi_{22} \bar{\rho}(\bar{\pi}-\tau)+\bar{\rho} \delta \Phi_{22}+\Phi_{22} \delta \bar{\rho}+\Phi \Gamma^{2}+\Gamma \delta \Phi+\Phi \delta \Gamma .
\end{aligned}
$$

Here the letter $\Phi$ is used to denote $\left\{\Phi_{20}, \Phi_{21}, \Phi_{11}\right\}$. The first term on the right hand side of the previous equation, $\delta^{2} \Phi_{21}$, can be controlled by

$$
\begin{aligned}
\int_{0}^{u}\left(\int_{\mathcal{S}_{u^{\prime}, v}}\left|\not \nabla \Phi_{11}\right|\left|\nabla^{2} \Phi_{21}\right|\right)^{1 / 2} \mathrm{~d} u^{\prime} & \leq \int_{0}^{u}\left(\left.\int_{\mathcal{S}_{u^{\prime}, v}}|\not| \Phi_{11}\right|^{2}\right)^{1 / 4}\left(\int_{\mathcal{S}_{u^{\prime}, v}}\left|\nabla^{2} \Phi_{21}\right|^{2}\right)^{1 / 4} \mathrm{~d} u^{\prime} \\
& \leq \sup _{u, v}\left\|\not \nabla \Phi_{11}\right\|_{L^{2}\left(\mathcal{S}_{u, v}\right)}^{1 / 2} \mid\left\|\nabla^{2} \Phi_{21}\right\|_{L^{2}\left(\mathcal{N}_{v}^{\prime}(0, u)\right)}^{1 / 2} \varepsilon^{3 / 4} \\
& \leq C\left(\Delta_{\Phi_{\star}}, \Delta_{\Phi}\right) \varepsilon^{3 / 4}
\end{aligned}
$$

In the case of the terms

$$
\Sigma_{2} \bar{\phi}_{2}(\bar{\pi}-\tau)+\bar{\phi}_{2} \delta \Sigma_{2}+\Sigma_{2} \delta \bar{\phi}_{2}+\Phi_{22} \bar{\rho}(\bar{\pi}-\tau)+\bar{\rho} \delta \Phi_{22}+\Phi_{22} \delta \bar{\rho},
$$

the use of the estimates of the curvature of the light cone (rather than on the sphere) gives a contribution with the same power of $\varepsilon$. Furthermore, the terms

$$
\Sigma_{4} \bar{\phi}_{3}(\tau-\bar{\pi}), \quad \text { and } \quad \Sigma_{4} \delta \bar{\phi}_{3}
$$

contribute with a power $\varepsilon^{5 / 4}$ since $\left\|\Sigma_{4}\right\|_{L^{\infty}\left(\mathcal{S}_{u, v}\right)}$ is controlled by $\varepsilon$ in Proposition 8 . For the term $\bar{\phi}_{3} \delta \Sigma_{4}$ we have that

$$
\begin{aligned}
& \int_{0}^{u}\left(\int_{\mathcal{S}_{u^{\prime}, v}}\left|\mathbb{\nabla} \Phi_{11} \| \bar{\phi}_{3} \not \nabla \Sigma_{4}\right|\right)^{1 / 2} \mathrm{~d} u^{\prime} \\
& \quad \leq \sup _{u, v}\left\|\mathbb{\nabla} \Phi_{11}\right\|_{L^{2}\left(\mathcal{S}_{u, v}\right)}^{1 / 2}\left\|\mathbb{\nabla} \Sigma_{4}\right\|_{L^{2}\left(\mathcal{S}_{u, v}\right)}^{1 / 2} \int_{0}^{u}\left\|\phi_{3}\right\|_{L^{\infty}\left(\mathcal{S}_{u^{\prime}, v}\right.}^{1 / 2} \mathrm{~d} u^{\prime} \\
& \quad \leq C\left(\Delta_{e_{\star}}\right) \sup _{u, v}\left\|\mathbb{\nabla} \Phi_{11}\right\|_{L^{2}\left(\mathcal{S}_{u, v}\right)}^{1 / 2}\left\|\not \nabla \Sigma_{4}\right\|_{L^{4}\left(\mathcal{S}_{u, v}\right)}^{1 / 2} \sum_{i=0}^{2}\left\|\mathbb{X}^{i} \phi_{3}\right\|_{L^{2}\left(\mathcal{N}_{v}^{\prime}(0, u)\right)}^{1 / 2} \varepsilon^{3 / 4} \\
& \leq C\left(I, \Delta_{e_{\star}}, \Delta_{\Gamma_{\star}}, \Delta_{\Sigma_{\star}}, \Delta_{\Phi_{\star}}, \Delta_{\phi}\right) \varepsilon^{5 / 4} .
\end{aligned}
$$


Here we have used the Sobolev inequality and Proposition 8. Next, the term $\Phi \Gamma^{2}$ gives us

$$
\begin{aligned}
& \int_{0}^{u}\left(\int_{\mathcal{S}_{u^{\prime}, v}}|\nabla| \Phi_{11} \| \Phi \Gamma^{2} \mid\right)^{1 / 2} \mathrm{~d} u^{\prime} \\
& \quad \leq \sum_{i=0}^{2} C\left(\Delta_{e_{\star}}\right) \sup _{u, v}\|\Gamma\|_{L^{\infty}\left(\mathcal{S}_{u, v}\right)}\left\|\not \nabla \Phi_{11}\right\|_{L^{2}\left(\mathcal{S}_{u, v}\right)}^{1 / 2}\left\|\nabla^{i} \Phi\right\|_{L^{2}\left(\mathcal{S}_{u, v}\right)}^{1 / 2} \varepsilon^{3 / 4} \\
& \quad \leq C\left(I, \Delta_{e_{\star}}, \Delta_{\Gamma_{\star}}, \Delta_{\Phi_{\star}}\right) \varepsilon^{3 / 4} .
\end{aligned}
$$

Terms $\Gamma \delta \Phi$ and $\Phi \delta \Gamma$ give a similar contribution. Putting everything together we find that

$$
\begin{aligned}
\left\|\mathbb{D} \Phi_{11}\right\|_{L^{2}\left(\mathcal{S}_{u, v}\right)} & \leq 2 \Delta_{\Phi_{\star}}+C\left(I, \Delta_{e_{\star}}, \Delta_{\Gamma_{\star}}, \Delta_{\Phi_{\star}}\right) \varepsilon^{3 / 4} \\
& +C\left(I, \Delta_{e_{\star}}, \Delta_{\Gamma_{\star}}, \Delta_{\Sigma_{\star}}, \Delta_{\Phi_{\star}}, \Delta_{\phi}\right) \varepsilon^{5 / 4}
\end{aligned}
$$

so that it is possible to choose a suitably small $\varepsilon_{\star}$ to improve the bootstrap assumption. Estimates for $\left\|\mathbb{Z}^{2}\left\{\Phi_{00}, \Phi_{01}, \Phi_{02}, \Phi_{11}, \Phi_{12}\right\}\right\|_{L^{2}\left(\mathcal{S}_{u, v}\right)}$. We present the analysis of $\not{ }^{2} \Phi_{11}$ as an example. The relevant short direction equation is

$$
\begin{aligned}
\Delta \delta^{2} \Phi_{11}= & \delta^{3} \Phi_{21}+\Phi \delta^{2} \Gamma+\Gamma \delta^{2} \Phi+\delta \Phi \delta \Gamma+\Phi \Gamma \delta \Gamma+\Gamma^{2} \delta \Phi \\
& +\Phi \Gamma^{3}+\Sigma \delta \phi+\phi \delta^{2} \Sigma+\delta \Sigma \delta \phi+\Sigma \Gamma \delta \phi+\phi \Gamma \delta \Sigma+\Sigma \phi \Gamma^{2}
\end{aligned}
$$

Then, making use of the short direction Grönwall-type estimate one obtains

$$
\begin{aligned}
& \left\|\mathbb{X}^{2} \Phi_{11}\right\|_{L^{2}\left(\mathcal{S}_{u, v}\right)} \\
& \quad \leq 2\left(\left\|\nabla^{2} \Phi_{11}\right\|_{L^{2}\left(\mathcal{S}_{0, v}\right)}+C\left(\Delta_{e_{\star}}, \Delta_{\Gamma_{\star}}\right) \int_{0}^{u}\left(\int_{\mathcal{S}_{u^{\prime}, v}} \Delta\left\langle\nabla^{2} \Phi_{11}, \nabla^{2} \Phi_{11}\right\rangle_{\sigma}\right)^{1 / 2} \mathrm{~d} u^{\prime}\right) \\
& \quad \leq 2 \Delta_{\Phi_{\star}}+C\left(\Delta_{e_{\star}}, \Delta_{\Gamma_{\star}}\right) \int_{0}^{u}\left(\int_{\mathcal{S}_{u^{\prime}, v}}\left|\nabla^{2} \Phi_{11}\right|\left(\left|\Delta T_{1}\right|+\left|\Delta T_{2}\right|\right)\right)^{1 / 2} \mathrm{~d} u^{\prime}
\end{aligned}
$$

where

$$
T_{1} \equiv \bar{\delta} \bar{\delta} \Phi_{11}+(\bar{\beta}-\alpha) \bar{\delta} \Phi_{11}, \quad T_{2} \equiv \bar{\delta} \delta \Phi_{11}+(\alpha-\bar{\beta}) \delta \Phi_{11}
$$

Since $\Phi$ contains only the components $\left\{\Phi_{11}, \Phi_{20}, \Phi_{21}, \Phi_{22}\right\}$, we can analyse terms which contain $\Phi$ in a similar way. Namely, we make use of the Hölder inequality to separate the product terms, and then we make use of the Sobolev embedding theorem. When we encounter the terms $\nabla^{i} \Phi_{22}$ and $\nabla^{3} \Phi_{21}$, we can make use of the estimate on the light cone. Finally, a quick inspection of the remaining terms reveals that only 
those related to $\Sigma_{2}$ contribute to the integration. For example, the term $\Sigma_{2} \delta \phi$ gives

$$
\begin{aligned}
& \int_{0}^{u}\left(\int_{\mathcal{S}_{u^{\prime}, v}}\left|\mathbb{X}^{2} \Phi_{11} \| \Sigma_{2} \delta \phi\right|\right)^{1 / 2} \mathrm{~d} u^{\prime} \\
& \quad \leq \int_{0}^{u}\left\|\nabla^{2} \Phi_{11}\right\|_{L^{2}\left(\mathcal{S}_{u^{\prime}, v}\right)}^{1 / 2}\left\|\Sigma_{2}\right\|_{L^{\infty}\left(\mathcal{S}_{u^{\prime}, v}\right)}^{1 / 2}\|\not \nabla \phi\|_{L^{2}\left(\mathcal{S}_{u^{\prime}, v}\right)}^{1 / 2} \mathrm{~d} u^{\prime} \\
& \quad \leq \sup _{u, v}\left\|\nabla^{2} \Phi_{11}\right\|_{L^{2}\left(\mathcal{S}_{u, v}\right)}^{1 / 2}\left\|\Sigma_{2}\right\|_{L^{\infty}\left(\mathcal{S}_{u, v}\right)}^{1 / 2}\|\not \nabla \phi\|_{L^{2}\left(\mathcal{N}_{v}^{\prime}(0, u)\right)}^{1 / 2} \varepsilon^{3 / 4} \\
& \quad \leq C\left(\Delta_{\Sigma_{\star}}, \Delta_{\Phi_{\star}}, \Delta_{\phi}\right) \varepsilon^{3 / 4} .
\end{aligned}
$$

Similarly, the Hölder and the Sobolev inequalities allow us to analyse other terms which are also controlled by $\varepsilon$. Putting everything together one finds that

$$
\sup _{u, v}\left\|\nabla^{2}\left\{\Phi_{00}, \Phi_{01}, \Phi_{02}, \Phi_{11}, \Phi_{12}\right\}\right\|_{L^{2}\left(\mathcal{S}_{u, v}\right)} \leq 3 \Delta_{\Phi_{\star}}
$$

Concluding the argument. From the estimates obtained in the previous paragraphs one concludes that

$$
\sup _{u, v}\left\|\mathbb{P}^{i}\left\{\Phi_{00}, \Phi_{01}, \Phi_{02}, \Phi_{11}, \Phi_{12}\right\}\right\|_{L^{2}\left(\mathcal{S}_{u, v}\right)} \leq 3 \Delta_{\Phi_{\star}}, \quad i=0, \ldots, 2 .
$$

Hence, we have improved the starting bootstrap assumption.

Using a similar method, we can obtain the following result:

Proposition 12 Assume that we are given a solution to the vacuum CEFEs in Stewart's gauge satisfying the same assumptions of Proposition 10. Then there exists

$$
\varepsilon_{\star}=\varepsilon_{\star}\left(I, \Delta_{e_{\star}}, \Delta_{\Gamma_{\star}}, \Delta_{\Sigma_{\star}}, \Delta_{\Phi_{\star}}, \Delta_{\Phi}, \Delta_{\phi}, \sup _{u, v}\left\|\mathbb{Z}^{3} \tau\right\|_{L^{2}\left(\mathcal{S}_{u, v}\right)}\right)
$$

such that for $\varepsilon \leq \varepsilon_{\star}$, we have

$$
\Delta_{\phi}(\mathcal{S})<3 \Delta_{\phi_{\star}}
$$

In order to estimate the curvature, we need $L^{2}\left(\mathcal{S}_{u, v}\right)$-estimates of the connection coefficients and the derivatives of the conformal factor up to third order. These estimates can be obtained, except for $\rho$ and $\sigma$, by a method similar to the one used in the previous proof. For these coefficients, instead of considering their $n$-direction equations, we make use of their long direction equations and the Codazzi equation to obtain the required estimates.

Proposition 13 (further control on the $L^{2}$-norm of the connection coefficients) Assume again that we have a solution of the vacuum CEFEs in Stewart's gauge in a region $\mathcal{D}_{u, v}^{t}$. with 


$$
\begin{aligned}
& \sup _{u, v}\left\|\left\{\mu, \lambda, \alpha, \beta, \epsilon, \rho, \sigma, \tau, \chi, \Sigma_{1}, \Sigma_{2}, \Sigma_{3}, \Sigma_{4}\right\}\right\|_{L^{\infty}\left(S_{u, v}\right)} \leq \infty, \\
& \sup _{u, v}\left\|\not \mathbb{X}\left\{\mu, \lambda, \alpha, \beta, \epsilon, \rho, \sigma, \Sigma_{1}, \Sigma_{2}, \Sigma_{3}, \Sigma_{4}\right\}\right\|_{L^{4}\left(\mathcal{S}_{u, v}\right)} \leq \infty, \\
& \sup _{u, v}\left\|\mathbb{X}^{2}\left\{\mu, \lambda, \alpha, \beta, \epsilon, \rho, \sigma, \tau, \Sigma_{1}, \Sigma_{2}, \Sigma_{3}, \Sigma_{4}\right\}\right\|_{L^{2}\left(\mathcal{S}_{u, v}\right)} \leq \infty, \\
& \Delta_{\Phi}(\mathcal{S})<\infty, \quad \Delta_{\Phi}<\infty, \quad \Delta_{\phi}(\mathcal{S})<\infty, \quad \Delta_{\phi}<\infty
\end{aligned}
$$

for some positive $\Delta_{\Gamma, \Sigma}$ and furthermore that

$$
\sup _{u, v}\left\|\mathbb{X}^{3}\left\{\mu, \lambda, \alpha, \beta, \epsilon, \tau, \Sigma_{1}, \Sigma_{2}, \Sigma_{3}, \Sigma_{4}\right\}\right\|_{L^{2}\left(\mathcal{S}_{u, v}\right)}<\infty
$$

on $\mathcal{D}_{u, v_{\bullet}}^{t}$. Then there exists $\varepsilon_{\star}=\varepsilon_{\star}\left(I, \Delta_{e_{\star}}, \Delta_{\Gamma_{\star}}, \Delta_{\Sigma_{\star}}, \Delta_{\Phi_{\star}}, \Delta_{\Phi}, \Delta_{\phi}\right)$ such that for $\varepsilon \leq \varepsilon_{\star}$, we have

$$
\begin{aligned}
& \sup _{u, v}\left\|\mathbb{X}^{3}\{\mu, \lambda, \alpha, \beta, \epsilon\}\right\|_{L^{2}\left(\mathcal{S}_{u, v}\right)} \leq 3 \Delta_{\Gamma_{\star}}, \\
& \sup _{u, v}\left\|\mathbb{X}^{3}\{\rho, \sigma\}\right\|_{L^{2}\left(\mathcal{S}_{u, v}\right)} \leq C\left(I, \Delta_{e_{\star}}, \Delta_{\Gamma_{\star}}, \Delta_{\Phi_{\star}}, \Delta_{\Phi}\right), \\
& \sup _{u, v}\left\|\mathbb{X}^{3}\{\tau, \chi\}\right\|_{L^{2}\left(\mathcal{S}_{u, v}\right)} \leq C\left(I, \Delta_{e_{\star}}, \Delta_{\Gamma_{\star}}, \Delta_{\Phi_{\star}}, \Delta_{\Phi}\right), \\
& \sup _{u, v}\left\|\mathbb{X}^{3} \Sigma_{2}\right\|_{L^{2}\left(\mathcal{S}_{u, v}\right)} \leq 3 \Delta_{\Sigma_{\star}}, \\
& \sup _{u, v}\left\|\mathbb{X}^{3}\left\{\Sigma_{1}, \Sigma_{3}, \Sigma_{4}\right\}\right\|_{L^{2}\left(\mathcal{S}_{u, v}\right)} \leq C\left(I, \Delta_{e_{\star}}, \Delta_{\Gamma_{\star}}, \Delta_{\Sigma_{\star}}, \Delta_{\Phi_{\star}}\right) \varepsilon .
\end{aligned}
$$

Proof Bootstrap assumption. We make the following bootstrap assumption to start the proof:

$$
\begin{aligned}
& \sup _{u, v}\left\|\mathbb{X}^{3}\{\mu, \lambda, \alpha, \beta, \epsilon\}\right\|_{L^{2}\left(\mathcal{S}_{u, v}\right)} \leq 4 \Delta_{\Gamma_{\star}}, \\
& \sup _{u, v}\left\|\mathbb{Z}^{3} \tau\right\|_{L^{2}\left(\mathcal{S}_{u, v}\right)} \leq \Delta_{\tau}, \\
& \sup _{u, v}\left\|\mathbb{X}^{3}\left\{\Sigma_{1}, \Sigma_{2}, \Sigma_{3}, \Sigma_{4}\right\}\right\|_{L^{2}\left(\mathcal{S}_{u, v}\right)} \leq 4 \Delta_{\Sigma_{\star}},
\end{aligned}
$$

where $\Delta_{\tau}$ is a constant whose value will be fixed later.

Estimates for $\rho$ and $\sigma$. We first estimate $\rho$ and $\sigma$ using the long direction Eqs. (13m) and (13f) as we want to avoid the higher derivatives on sphere in the short direction equations. From the full expression of $\left\|\nabla^{3} \rho\right\|_{L^{2}\left(\mathcal{S}_{u, v}\right)}$ (see Appendix C in Paper I), we will analyse four typical terms namely, $\delta^{3} \rho, \xi \delta^{2} \rho, \delta \xi \delta \rho$ and $\xi^{2} \delta \rho$. For the term $\delta^{3} \rho$, we have

$$
\begin{aligned}
D \delta^{3} \rho= & \Gamma^{5}+\Gamma^{3} \delta \Gamma+\Gamma(\delta \Gamma)^{2}+\Gamma^{2} \delta^{2} \Gamma+\delta \Gamma \delta^{2} \Gamma+\rho \delta^{3}(\epsilon+\bar{\epsilon}) \\
& +(4 \epsilon-2 \bar{\epsilon}+5 \rho) \delta^{3} \rho+\sigma \delta^{3} \bar{\sigma}+\bar{\sigma} \delta^{3} \sigma+\sigma \delta^{2} \bar{\delta} \rho+\delta^{3} \Phi_{00}
\end{aligned}
$$


The term $\delta \Gamma \delta^{2} \Gamma$ can be estimated as

$$
\begin{aligned}
\left\|\delta \Gamma \delta^{2} \Gamma\right\|_{L^{2}\left(\mathcal{S}_{u, v}\right)} & \leq\|\mathbb{\nabla} \Gamma\|_{L^{4}\left(\mathcal{S}_{u, v}\right)}\left\|\mathbb{X}^{2} \Gamma\right\|_{L^{4}\left(\mathcal{S}_{u, v}\right)} \\
& \leq C\left(\Delta_{e_{\star}}\right)\|\mathbb{X} \Gamma\|_{L^{4}\left(\mathcal{S}_{u, v}\right)}\left(\left\|\mathbb{Z}^{2} \Gamma\right\|_{L^{2}\left(\mathcal{S}_{u, v}\right)}+\left\|\mathbb{Z}^{3} \Gamma\right\|_{L^{2}\left(\mathcal{S}_{u, v}\right)}\right),
\end{aligned}
$$

where $\Gamma$ contains $\epsilon, \rho$ and $\sigma$. Then, making use of the norm of $\Phi_{00}$ on the long light cone, we find that

$$
\begin{aligned}
\int_{0}^{v}\left\|\delta^{3} \Phi_{00}\right\|_{L^{2}\left(\mathcal{S}_{u, v^{\prime}}\right)} \mathrm{d} v^{\prime} & \leq\left(\int_{0}^{v} \int_{\mathcal{S}_{u, v^{\prime}}}\left|\delta^{3} \Phi_{00}\right|^{2} \mathrm{~d} v^{\prime}\right)^{1 / 2}\left(\int_{0}^{v} 1 \mathrm{~d} v^{\prime}\right)^{1 / 2} \\
& \leq C(I)\left\|\mathbb{X}^{3} \Phi_{00}\right\|_{L^{2}\left(\mathcal{N}_{u}(0, v)\right)}
\end{aligned}
$$

Hence, the long direction of inequality in Proposition 3 yields

$$
\begin{aligned}
\left\|\delta^{3} \rho\right\|_{L^{2}\left(\mathcal{S}_{u, v}\right) \leq} & C\left(I, \Delta_{e_{\star}}, \Delta_{\Gamma_{\star}}, \Delta_{\Phi_{\star}}, \Delta_{\Phi}\right) \\
& +C\left(I, \Delta_{\Gamma_{\star}}\right) \int_{0}^{v}\left(\left\|\nabla^{3} \rho\right\|_{L^{2}\left(\mathcal{S}_{u, v^{\prime}}\right)}+\left\|\mathbb{D}^{3} \sigma\right\|_{L^{2}\left(\mathcal{S}_{u, v^{\prime}}\right)}\right) \mathrm{d} v^{\prime}
\end{aligned}
$$

For the term $\varpi \delta^{2} \rho$, we readily find that

$$
\left\|\varpi \delta^{2} \rho\right\|_{L^{2}\left(\mathcal{S}_{u, v}\right)} \leq\|\varpi\|_{L^{\infty}\left(\mathcal{S}_{u, v}\right)}\left\|\nabla^{2} \rho\right\|_{L^{2}\left(\mathcal{S}_{u, v}\right)} \leq C\left(\Delta_{\Gamma_{\star}}\right) .
$$

Similar estimates can be found for $\delta \varpi \delta \rho$ and $\varpi^{2} \delta \rho$. Hence, we conclude that

$$
\begin{aligned}
\left\|\nabla^{3} \rho\right\|_{L^{2}\left(\mathcal{S}_{u, v}\right) \leq} & C\left(I, \Delta_{e_{\star}}, \Delta_{\Gamma_{\star}}, \Delta_{\Phi_{\star}}, \Delta_{\Phi}\right) \\
& +C\left(I, \Delta_{\Gamma_{\star}}\right) \int_{0}^{v}\left(\left\|\mathbb{D}^{3} \rho\right\|_{L^{2}\left(\mathcal{S}_{u, v^{\prime}}\right)}+\left\|\mathbb{D}^{3} \sigma\right\|_{L^{2}\left(\mathcal{S}_{u, v^{\prime}}\right)}\right) \mathrm{d} v^{\prime}
\end{aligned}
$$

From here, using Grönwall's inequality one finds that

$$
\left\|\nabla^{3} \rho\right\|_{L^{2}\left(\mathcal{S}_{u, v}\right)} \leq C\left(I, \Delta_{e_{\star}}, \Delta_{\Gamma_{\star}}, \Delta_{\Phi_{\star}}, \Delta_{\Phi}\right)+C\left(I, \Delta_{\Gamma_{\star}}\right) \int_{0}^{v}\left\|\nabla^{3} \sigma\right\|_{L^{2}\left(\mathcal{S}_{u, v^{\prime}}\right)} \mathrm{d} v^{\prime}
$$

In order to estimate $\left\|\mathbb{X}^{3} \sigma\right\|_{L^{2}\left(\mathcal{S}_{u, v}\right)}$, we need to control the third order derivatives of $\sigma$ for example $\left\|\delta^{3} \sigma\right\|_{L^{2}\left(\mathcal{S}_{u, v}\right)}$. Using integration by parts and the structure equation (Codazzi equation) (13q) one finds that

$$
\begin{aligned}
\left\|\delta^{3} \sigma\right\|_{L^{2}\left(\mathcal{S}_{u, v}\right) \leq} & \left\|\delta^{3} \rho\right\|_{L^{2}\left(\mathcal{S}_{u, v}\right)}+C\left(I, \Delta_{e_{\star}}, \Delta_{\Gamma_{\star}}, \Delta_{\Phi_{\star}}\right)+\left\|\nabla^{2} \Phi_{01}\right\|_{L^{2}\left(\mathcal{S}_{u, v}\right)} \\
& +C\left(I, \Delta_{e_{\star}}, \Delta_{\Gamma_{\star}}, \Delta_{\Sigma_{\star}}, \Delta_{\Phi_{\star}}\right) \sum_{i=0}^{3}\left\|\nabla^{i} \phi_{0}\right\|_{L^{2}\left(\mathcal{S}_{u, v}\right)} \varepsilon
\end{aligned}
$$


so that, in fact, one has

$$
\begin{aligned}
\left\|\nabla^{3} \rho\right\|_{L^{2}\left(\mathcal{S}_{u, v}\right) \leq} & C\left(I, \Delta_{e_{\star}}, \Delta_{\Gamma_{\star}}, \Delta_{\Phi_{\star}}, \Delta_{\Phi}\right)+C\left(I, \Delta_{e_{\star}}, \Delta_{\Gamma_{\star}}, \Delta_{\Sigma_{\star}}, \Delta_{\Phi_{\star}}, \Delta_{\phi}\right) \varepsilon \\
& +C\left(I, \Delta_{\Gamma_{\star}}\right) \int_{0}^{v}\left\|\mathbb{X}^{3} \rho\right\|_{L^{2}\left(\mathcal{S}_{u, v^{\prime}}\right)} \mathrm{d} v^{\prime}
\end{aligned}
$$

Now, using Grönwall's inequality one concludes that

$$
\left\|\mathbb{D}^{3} \rho\right\|_{L^{2}\left(\mathcal{S}_{u, v}\right)} \leq C\left(I, \Delta_{e_{\star}}, \Delta_{\Gamma_{\star}}, \Delta_{\Phi_{\star}}, \Delta_{\Phi}\right)+C\left(I, \Delta_{e_{\star}}, \Delta_{\Gamma_{\star}}, \Delta_{\Sigma_{\star}}, \Delta_{\Phi_{\star}}, \Delta_{\phi}\right) \varepsilon
$$

so that $\left\|\mathbb{X}^{3} \rho\right\|_{L^{2}(S)_{u, v}}$ is bounded. Moreover, one has that

$$
\left\|\mathbb{P}^{3} \sigma\right\|_{L^{2}\left(\mathcal{S}_{u, v}\right)} \leq C\left(I, \Delta_{e_{\star}}, \Delta_{\Gamma_{\star}}, \Delta_{\Phi_{\star}}, \Delta_{\Phi}\right)+C\left(I, \Delta_{e_{\star}}, \Delta_{\Gamma_{\star}}, \Delta_{\Sigma_{\star}}, \Delta_{\Phi_{\star}}, \Delta_{\phi}\right) \varepsilon
$$

Estimates for $\tau$ and $\chi$. The $\Delta$-equation for $\nabla^{3} \tau$ can be obtained from the structure Eq. (13b) and the commutator relationship. More precisely, one has that

$$
\begin{aligned}
D \delta^{3} \tau= & \delta^{3}\left(\Xi \phi_{1}\right)+\delta^{3} \Phi_{01}+\Gamma \delta^{3} \Gamma_{1}+\Gamma \delta^{3} \tau+\Gamma \delta^{2} \Psi_{1} \\
& +\delta \Gamma \delta^{2} \Gamma+\Gamma^{2} \delta^{2} \Gamma+\Gamma^{3} \delta \Gamma+\Gamma(\delta \Gamma)^{2},
\end{aligned}
$$

where $\Gamma_{1}$ contains $\epsilon, \alpha, \beta, \rho$ and $\sigma$. Then, using the bootstrap assumption and the definition of $\Delta_{\Psi}$, we obtain

$$
\begin{aligned}
\left\|\mathbb{X}^{3} \tau\right\|_{L^{2}\left(\mathcal{S}_{u, v}\right) \leq} & C\left(I, \Delta_{e_{\star}}, \Delta_{\Gamma_{\star}}, \Delta_{\Phi_{\star}}, \Delta_{\Phi}\right)+C\left(I, \Delta_{e_{\star}}, \Delta_{\Sigma_{\star}}, \Delta_{\Gamma_{\star}}, \Delta_{\Phi_{\star}}, \Delta_{\phi}\right) \varepsilon \\
& +C\left(I, \Delta_{\Gamma_{\star}}\right) \int_{0}^{v}\left\|\mathbb{X}^{3} \tau\right\|_{L^{2}\left(\mathcal{S}_{u, v^{\prime}}\right)} \mathrm{d} v^{\prime}
\end{aligned}
$$

so that using Grönwall's inequality we conclude that

$$
\|\left.\mathbb{P}^{3} \tau\right|_{L^{2}\left(\mathcal{S}_{u, v}\right)} \leq C\left(I, \Delta_{e_{\star}}, \Delta_{\Gamma_{\star}}, \Delta_{\Phi_{\star}}, \Delta_{\Phi}\right)+C\left(\Delta_{e_{\star}}, \Delta_{\Sigma_{\star}}, \Delta_{\Gamma_{\star}}, \Delta_{\Phi_{\star}}, \Delta_{\phi}\right) \varepsilon .
$$

We can then choose the constant $\Delta_{\tau}$ larger than the right side above so as to improve the bootstrap assumption. The estimate of $\chi$ is similar:

$$
\left\|\mathbb{P}^{3} \chi\right\|_{L^{2}\left(\mathcal{S}_{u, v}\right)} \leq C\left(I, \Delta_{e_{\star}}, \Delta_{\Gamma_{\star}}, \Delta_{\Phi_{\star}}, \Delta_{\Phi}\right)+C\left(\Delta_{e_{\star}}, \Delta_{\Sigma_{\star}}, \Delta_{\Gamma_{\star}}, \Delta_{\Phi_{\star}}, \Delta_{\phi}\right) \varepsilon .
$$

Estimates for the the remaining spin connection coefficients. To obtain the estimates for

$$
\left\|\mathbb{Z}^{3}\{\mu, \lambda, \alpha, \beta, \epsilon\}\right\|_{L^{2}\left(\mathcal{S}_{u, v}\right)},
$$

we make use of their short direction equations. Since the proof are similar, we only show the details of $\epsilon$ as a representative example. In this case the relevant equation is

$$
\Delta \delta^{3} \epsilon=-\delta^{3}\left(\Xi \phi_{2}+\Phi_{12}\right)+\Gamma \delta^{3} \Gamma_{1}+\Gamma \delta^{3} \epsilon+\delta \Gamma \delta^{2} \Gamma+\Gamma^{2} \delta^{2} \Gamma+\Gamma^{3} \delta \Gamma+\Gamma(\delta \Gamma)^{2}+\Gamma^{5},
$$


where $\Gamma_{1}$ does not contain $\epsilon$. We can then make use of the short inequality in Proposition 3 and obtain that

$$
\left\|\nabla^{3} \epsilon\right\|_{L^{2}\left(\mathcal{S}_{u, v}\right)} \leq 2 \Delta_{\Gamma_{\star}}+C\left(I, \Delta_{e_{\star}}, \Delta_{\Gamma_{\star}}, \Delta_{\Phi_{\star}}, \Delta_{\Phi}\right) \varepsilon^{3 / 4}+o\left(\varepsilon^{3 / 4}\right) .
$$

Choosing the integral range sufficiently small we conclude that

$$
\left\|\nabla^{3} \epsilon\right\|_{L^{2}\left(\mathcal{S}_{u, v}\right)} \leq 3 \Delta_{\Gamma_{\star}}
$$

The estimates of $\left\|\nabla^{3}\{\mu, \lambda, \alpha, \beta\}\right\|_{L^{2}\left(\mathcal{S}_{u, v}\right)}$ are similar. Hence, we have improved the bootstrap assumption for the connection coefficients.

Estimates for $\nabla^{3} \Sigma_{2}$. The short direction equation for $\delta^{3} \Sigma_{2}$ can be analysed by the same method. Starting from

$$
\begin{aligned}
\Delta \delta^{3} \Sigma_{2}= & \Gamma^{3} \delta \Sigma_{2}+\Gamma \delta \Sigma_{2} \delta \Gamma+\Gamma^{2} \delta^{2} \Sigma_{2}+\delta \Gamma \delta^{2} \Sigma_{2}+\delta \Sigma_{2} \delta^{2} \Gamma+\Gamma \delta^{3} \Sigma_{2} \\
& +\sum_{i_{1}+\cdots+i_{4}=3} \delta^{i_{1}} \Xi \delta^{i_{2}} \Gamma^{i_{3}} \delta^{i_{4}} \Phi_{22},
\end{aligned}
$$

where $\Gamma$ contains $\tau$ and it is observed that the terms in the summation will contribute higher order of $\varepsilon$ in the integration. Then applying Proposition 11 we find that

$$
\left\|\nabla^{3} \Sigma_{2}\right\|_{L^{2}\left(\mathcal{S}_{u, v}\right)} \leq 2 \Delta_{\Sigma_{\star}}+C\left(I, \Delta_{e_{\star}}, \Delta_{\Gamma_{\star}}, \Delta_{\Sigma_{\star}}, \Delta_{\Phi_{\star}}\right) \varepsilon+o(\varepsilon)
$$

where the term $o(\varepsilon)$ arises from the summation.

Estimates for $\not^{3} \Sigma_{1}$. In this case one has that the $\Delta$-equation for $\Delta \delta^{3} \Sigma_{1}$ is of the form

$$
\begin{aligned}
\Delta \delta^{3} \Sigma_{1}= & \Sigma_{2} \Phi \Gamma^{2}+\Phi \Gamma \not \nabla \Sigma_{2}+\not \nabla \Sigma_{2} \not \nabla \Phi+\Sigma_{2} \Phi \not \Gamma+\Phi \not{ }^{2} \Sigma_{2} \\
& +s \Gamma^{3}+s \Gamma \delta \Gamma+s \delta^{2} \Gamma+\sum_{i_{1}+\cdots+i_{4}=3} \delta^{i_{1}} \Xi \delta^{i_{2}} \Gamma^{i_{3}} \delta^{i_{4}} \Phi
\end{aligned}
$$

here the first line on the right hand side contains the leading order contribution, and $\Phi$ does not contain $\Phi_{22}$. From this equation one readily obtains that

$$
\left\|\mathbb{Z}^{3} \Sigma_{1}\right\|_{L^{2}\left(\mathcal{S}_{u, v}\right)} \leq C\left(I, \Delta_{e_{\star}}, \Delta_{\Gamma_{\star}}, \Delta_{\Sigma_{\star}}, \Delta_{\Phi_{\star}}\right) \varepsilon+o(\varepsilon) .
$$

Estimates for $\nabla^{3} \Sigma_{3,4}$. In this case the term contributing to the leading order of the estimate of $\left\|\mathbb{X}^{3} \Sigma_{3}\right\|_{L^{2}\left(\mathcal{S}_{u, v}\right)}$ is

$$
\begin{aligned}
\Delta \delta^{3} \Sigma_{3}= & \Sigma_{2} \Gamma^{4}+\Gamma \not \Sigma_{2}+\Sigma_{2} \Gamma^{2} \not \Gamma+\Sigma_{2}(\not \Gamma)^{2}+\Gamma^{2} \not{ }^{2} \Sigma_{2}+\Sigma_{2} \Gamma \not^{2} \Gamma \\
& +\Sigma_{2} \not{ }^{3} \Gamma+\Gamma \not{ }^{3} \Sigma_{2} \\
& +\sum_{i_{1}+\cdots+i_{4}=3} \delta^{i_{1}} \Xi \delta^{i_{2}} \Gamma^{i_{3}} \delta^{i_{4}} \Phi,
\end{aligned}
$$


again here the first line of the right hand side offers the leading contribution, and gives

$$
\left\|\mathbb{P}^{3} \Sigma_{3}\right\|_{L^{2}\left(\mathcal{S}_{u, v}\right)} \leq C\left(I, \Delta_{e_{\star}}, \Delta_{\Gamma_{\star}}, \Delta_{\Sigma_{\star}}, \Delta_{\Phi_{\star}}\right) \varepsilon+o(\varepsilon)
$$

Concluding the argument. From the analysis above, it follows that we can choose

$$
\varepsilon_{\star}=\varepsilon_{\star}\left(I, \Delta_{e_{\star}}, \Delta_{\Gamma_{\star}}, \Delta_{\Sigma_{\star}}, \Delta_{\phi_{\star}}, \Delta_{\Phi_{\star}}, \Delta_{\phi}, \Delta_{\Phi}\right),
$$

sufficiently small so that

$$
\begin{aligned}
& \sup _{u, v}\left\|\mathbb{D}^{3}\{\mu, \lambda, \alpha, \beta, \epsilon\}\right\|_{L^{2}\left(\mathcal{S}_{u, v}\right)} \leq 3 \Delta_{\Gamma_{\star}}, \\
& \sup _{u, v}\left\|\mathbb{X}^{3}\{\rho, \sigma\}\right\|_{L^{2}\left(\mathcal{S}_{u, v}\right)} \leq C\left(I, \Delta_{e_{\star}}, \Delta_{\Gamma_{\star}}, \Delta_{\Phi_{\star}}, \Delta_{\Phi}\right), \\
& \sup _{u, v}\left\|\mathbb{X}^{3}\{\tau, \chi\}\right\|_{L^{2}\left(\mathcal{S}_{u, v}\right)} \leq C\left(I, \Delta_{e_{\star}}, \Delta_{\Gamma_{\star}}, \Delta_{\Phi_{\star}}, \Delta_{\Phi}\right), \\
& \sup _{u, v}\left\|\mathbb{X}^{3} \Sigma_{2}\right\|_{L^{2}\left(\mathcal{S}_{u, v}\right)} \leq 3 \Delta_{\Sigma_{\star}}, \\
& \sup _{u, v}\left\|\mathbb{X}^{3}\left\{\Sigma_{1}, \Sigma_{3}, \Sigma_{4}\right\}\right\|_{L^{2}\left(\mathcal{S}_{u, v}\right)} \leq C\left(I, \Delta_{e_{\star}}, \Delta_{\Gamma_{\star}}, \Delta_{\Sigma_{\star}}, \Delta_{\Phi_{\star}}\right) \varepsilon,
\end{aligned}
$$

on $\mathcal{D}_{u, v_{\bullet}}^{t}$.

\subsection{The energy estimates for the curvature}

In this subsection, we show how to obtain the main energy estimates for the components of the Ricci and rescaled Weyl curvature.

\subsubsection{Analysis of the rescaled Weyl tensor}

We begin by introducing some integral identities which follow from using integration by parts in the conformal equations satisfied by the components of the rescaled Weyl tensor, Eqs. (21a)-(21h). The proof of these results follows the same arguments used for the components of the Weyl tensor in Paper I as the (vacuum) Bianchi identities have an identical structure to that of the equations for the rescaled Weyl tensor and are thus omitted.

Proposition 14 (control of the angular derivatives of the components of the rescaled Weyl tensor) Suppose that we are given a solution to the CEFEs in Stewart's gauge and that $\mathcal{D}_{u, v}$ is contained in the existence area. The following $L^{2}$ estimates for the components of the rescaled Weyl curvature hold. First,

$$
\begin{aligned}
& \sum_{i=0,1,2} \int_{\mathcal{N}_{u}(0, v)}\left|\phi_{i}\right|^{2}+\sum_{j=1,2,3} \int_{\mathcal{N}_{v}^{\prime}(0, u)} Q^{-1}\left|\phi_{j}\right|^{2} \\
& \leq \sum_{i=0,1,2} \int_{\mathcal{N}_{0}(0, v)}\left|\phi_{i}\right|^{2}+\sum_{j=1,2,3} \int_{\mathcal{N}_{0}^{\prime}(0, u)} Q^{-1}\left|\phi_{j}\right|^{2}+\int_{\mathcal{D}_{u, v}} \phi_{H} \phi \Gamma,
\end{aligned}
$$


then

$$
\begin{aligned}
& \sum_{i=0,1,2} \int_{\mathcal{N}_{u}(0, v)}\left|\not \nabla \phi_{i}\right|^{2}+\sum_{j=1,2,3} \int_{\mathcal{N}_{v}^{\prime}(0, u)} Q^{-1}\left|\not \nabla \phi_{j}\right|^{2} \\
& \leq \sum_{i=0,1,2} \int_{\mathcal{N}_{0}(0, v)}\left|\mathbb{\nabla} \phi_{i}\right|^{2}+\sum_{j=1,2,3} \int_{\mathcal{N}_{0}^{\prime}(0, u)} Q^{-1}\left|\not \nabla \phi_{j}\right|^{2} \\
& \quad+\int_{\mathcal{D}_{u, v}}\left|\mathbb{\nabla} \phi_{H}\right|\left(\phi \Gamma^{2}+\Gamma|\not \nabla \phi|+\phi|\not| \Gamma \mid\right),
\end{aligned}
$$

next

$$
\begin{aligned}
& \sum_{i=0,1,2} \int_{\mathcal{N}_{u}(0, v)}\left|\nabla^{2} \phi_{i}\right|^{2}+\sum_{j=1,2,3} \int_{\mathcal{N}_{v}^{\prime}(0, u)} Q^{-1}\left|\nabla^{2} \phi_{j}\right|^{2} \\
& \leq \sum_{i=0,1,2} \int_{\mathcal{N}_{0}(0, v)}\left|\mathbb{Z}^{2} \phi_{i}\right|^{2}+\sum_{j=1,2,3} \int_{\mathcal{N}_{0}^{\prime}(0, u)} Q^{-1}\left|\nabla^{2} \phi_{j}\right|^{2} \\
& \quad+\int_{\mathcal{D}_{u, v}}\left|\nabla^{2} \phi_{H}\right|\left(\Gamma\left|\nabla^{2} \phi\right|+\phi\left|\nabla^{2} \Gamma\right|+|\not \nabla \phi||\not| \Gamma\left|+\Gamma^{2}\right| \not|\phi|+\phi \Gamma|\not| \Gamma \mid+\Gamma^{3} \phi\right),
\end{aligned}
$$

and finally

$$
\begin{aligned}
& \sum_{i=0,1,2} \int_{\mathcal{N}_{u}(0, v)}\left|\nabla^{3} \phi_{i}\right|^{2}+\sum_{j=1,2,3} \int_{\mathcal{N}_{v}^{\prime}(0, u)} Q^{-1}\left|\nabla^{3} \phi_{j}\right|^{2} \\
& \leq \sum_{i=0,1,2} \int_{\mathcal{N}_{0}(0, v)}\left|\mathbb{Z}^{3} \phi_{i}\right|^{2}+\sum_{j=1,2,3} \int_{\mathcal{N}_{0}^{\prime}(0, u)} Q^{-1}\left|\nabla^{3} \phi_{j}\right|^{2}+\int_{\mathcal{D}_{u, v}}\left|\mathbb{Z}^{3} \phi_{H}\right|\left(\Gamma\left|\nabla^{3} \phi\right|\right.
\end{aligned}
$$

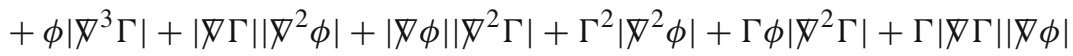

$$
\begin{aligned}
& \left.+\left.\phi|\not| \Gamma\right|^{2}+\Gamma^{3}|\not \nabla \phi|+\phi \Gamma^{2}|\not| \Gamma \mid+\Gamma^{4} \phi\right),
\end{aligned}
$$

where $\Gamma$ stands for arbitrary connection coefficients from the collection $\{\mu, \lambda, \alpha$, $\beta, \epsilon, \rho, \sigma, \tau\}$.

To summarise, the previous results can be given a more general formulation:

Proposition 15 Suppose that we are given a solution to the CEFEs in Stewart's gauge and that $\mathcal{D}_{u, v}$ is contained in the existence area. Then we have that

$$
\begin{aligned}
& \sum_{i=0,1,2} \int_{\mathcal{N}_{u}(0, v)}\left|\mathbb{X}^{m} \phi_{i}\right|^{2}+\sum_{j=1,2,3} \int_{\mathcal{N}_{v}^{\prime}(0, u)} Q^{-1}\left|\mathbb{X}^{m} \phi_{j}\right|^{2} \\
& \leq \sum_{i=0,1,2} \int_{\mathcal{N}_{0}(0, v)}\left|\mathbb{X}^{m} \phi_{i}\right|^{2}+\sum_{j=1,2,3} \int_{\mathcal{N}_{0}^{\prime}(0, u)} Q^{-1}\left|\nabla^{m} \phi_{j}\right|^{2} \\
& \quad+\int_{\mathcal{D}_{u, v}}\left|\mathbb{X}^{m} \phi_{H}\right| \sum_{i_{1}+i_{2}+i_{3}+i_{4}=m}\left|\mathbb{X}^{i_{1}} \Gamma^{i_{2}}\right|\left|\mathbb{X}^{i_{3}} \Gamma\right|\left|\mathbb{X}^{i_{4}} \phi\right|,
\end{aligned}
$$


where $\phi$ contains $\phi_{k}, k=0, \ldots, 4, \phi_{H}$ contains $\phi_{k}, k=0, \ldots, 3$.

In addition, we have the following proposition:

Proposition 16 (control of the angular derivatives of the "bad" components of the rescaled Weyl tensor) Suppose that we are given a solution to the CEFEs in Stewart's gauge and that $\mathcal{D}_{u, v}$ is contained in the existence area. Then we have that

$$
\begin{aligned}
& \int_{\mathcal{N}_{u}(0, v)}\left|\nabla^{m} \phi_{3}\right|^{2}+\int_{\mathcal{N}_{v}^{\prime}(0, u)} Q^{-1}\left|\nabla^{m} \phi_{4}\right|^{2} \\
& \leq \int_{\mathcal{N}_{0}(0, v)}\left|\nabla^{m} \phi_{3}\right|^{2}+\int_{\mathcal{N}_{0}^{\prime}(0, u)} Q^{-1}\left|\nabla^{m} \phi_{4}\right|^{2} \\
& +\int_{\mathcal{D}_{u, v}}\left|\nabla^{m} \phi_{4}\right| \sum_{i_{1}+i_{2}+i_{3}+i_{4}=m}\left|\mathbb{X}^{i_{1}} \Gamma^{i_{2}}\right|\left|\mathbb{X}^{i_{3}}(\rho+\epsilon)\right|\left|\mathbb{X}^{i_{4}} \phi_{4}\right| \\
& +\int_{\mathcal{D}_{u, v}}\left|\mathbb{X}^{m} \phi_{3}\right| \sum_{i_{1}+i_{2}+i_{3}+i_{4}=m}\left|\mathbb{X}^{i_{1}} \Gamma^{i_{2}}\right|\left|\mathbb{X}^{i_{3}} \Gamma\right|\left|\mathbb{X}^{i_{4}} \phi\right| \\
& +\int_{\mathcal{D}_{u, v}}\left|\mathbb{X}^{m} \phi_{4}\right| \sum_{i_{1}+i_{2}+i_{3}+i_{4}=m}\left|\mathbb{X}^{i_{1}} \Gamma^{i_{2}}\right|\left|\mathbb{X}^{i_{3}} \Gamma\right|\left|\mathbb{X}^{i_{4}} \phi_{H}^{\prime}\right|,
\end{aligned}
$$

where $\phi$ contains $\phi_{3}$ and $\phi_{4}, \phi_{H}^{\prime}$ contains $\phi_{2}$ and $\phi_{3}$.

\subsubsection{Analysis of the Ricci curvature}

In order to estimate the $L^{2}$-norms of the components of the Ricci tensor we need inequalities analogous to the ones used for the rescaled Weyl tensor. In order to obtain these, we first need to regroup the conformal equations for the Ricci tensor shown in Appendix A.2.3. More precisely, we pair the components $\Phi_{01}$ and $\Phi_{11}$ by analysing Eqs. (19b) and (19h); pair the components $\Phi_{02}$ and $\Phi_{12}$ by analysing (19c) and (19g)+(19l); pair the components $\Phi_{11}$ and $\Phi_{12}$ by analysing (19d) and (19g); pair the components $\Phi_{01}$ and $\Phi_{02}$ by analysing (19b)+(191) and (19h). Making use of this strategy one obtains the following:

Proposition 17 Suppose that we are given a solution to the CEFEs in Stewart's gauge and that $\mathcal{D}_{u, v}$ is contained in the existence area. Then we have that

$$
\begin{aligned}
& \sum_{\Phi_{i} \in \Phi_{L}} \int_{\mathcal{N}_{u}(0, v)}\left|\Phi_{i}\right|^{2}+\sum_{\Phi_{j} \in \Phi_{S}} \int_{\mathcal{N}_{v}^{\prime}(0, u)} Q^{-1}\left|\Phi_{j}\right|^{2} \\
& \leq \sum_{\Phi_{i} \in \Phi_{L}} \int_{\mathcal{N}_{0}(0, v)}\left|\Phi_{i}\right|^{2}+\sum_{\Phi_{j} \in \Phi_{S}} \int_{\mathcal{N}_{0}^{\prime}(0, u)} Q^{-1}\left|\Phi_{j}\right|^{2} \\
& \quad+\int_{\mathcal{D}_{u, v}} \Phi_{H} \Gamma \Phi+\int_{\mathcal{D}_{u, v}} \Phi_{H} \Sigma \phi
\end{aligned}
$$

where $\Phi_{L}=\equiv\left\{\Phi_{00}, \Phi_{01}, \Phi_{02}, \Phi_{11}\right\}, \Phi_{S} \equiv\left\{\Phi_{01}, \Phi_{02}, \Phi_{11}, \Phi_{12}\right\}, \Phi \equiv\left\{\Phi_{00}, \Phi_{01}\right.$, $\left.\Phi_{02}, \Phi_{11}, \Phi_{12}, \Phi_{22}\right\}$ and $\Phi_{H} \equiv\left\{\Phi_{00}, \Phi_{01}, \Phi_{02}, \Phi_{11}, \Phi_{12}\right\}$. 
Proof For simplicity, we demonstrate the argument with the conformal Eqs. (19a) and $(19 \mathrm{j})$ written in the form

$$
\begin{aligned}
& \Delta \Phi_{00}=\delta \Phi_{10}+\Gamma \Phi+\Sigma \phi, \\
& D \Phi_{01}=\delta \Phi_{00}+\Gamma \Phi+\Sigma \phi .
\end{aligned}
$$

Integrating by parts we have that

$$
\begin{aligned}
\int_{\mathcal{N}_{u}(0, v)}\left|\Phi_{00}\right|^{2}+\int_{\mathcal{N}_{v}^{\prime}(0, u)} Q^{-1}\left|\Phi_{01}\right|^{2} \leq & \int_{\mathcal{N}_{0}(0, v)}\left|\Phi_{00}\right|^{2}+\int_{\mathcal{N}_{0}^{\prime}(0, u)} Q^{-1}\left|\Phi_{01}\right|^{2} \\
& +\int_{\mathcal{D}_{u, v}}\left(\Phi_{00}, \Phi_{01}\right) \Gamma \Phi+\int_{\mathcal{D}_{u, v}}\left(\Phi_{00}, \Phi_{01}\right) \Sigma \phi .
\end{aligned}
$$

A similar argument applies to the pairs $\Phi_{01}$ and $\Phi_{11}, \Phi_{02}$ and $\Phi_{12}, \Phi_{11}$ and $\Phi_{12}, \Phi_{01}$ and $\Phi_{02}$. Putting everything together we obtain the required result.

Now, applying the angular derivatives to the conformal equations we obtain the following statement:

Proposition 18 Suppose that we are given a solution to the CEFEs in Stewart's gauge and that $\mathcal{D}_{u, v}$ is contained in the existence area. Then we have first that

$$
\begin{aligned}
& \sum_{\Phi_{i} \in \Phi_{L}} \int_{\mathcal{N}_{u}(0, v)}\left|\not \nabla \Phi_{i}\right|^{2}+\left.\sum_{\Phi_{j} \in \Phi_{S}} \int_{\mathcal{N}_{v}^{\prime}(0, u)} Q^{-1}|\not| \Phi_{j}\right|^{2} \\
& \leq \sum_{\Phi_{i} \in \Phi_{L}} \int_{\mathcal{N}_{0}(0, v)}\left|\not \nabla \Phi_{i}\right|^{2}+\sum_{\Phi_{j} \in \Phi_{S}} \int_{\mathcal{N}_{0}^{\prime}(0, u)} Q^{-1}\left|\not \nabla \Phi_{j}\right|^{2} \\
& +\int_{\mathcal{D}_{u, v}}\left|\not \nabla \Phi_{H}\right|\left(\Phi \Gamma^{2}+\Gamma|\not| \nabla|+\Phi| \not|\Gamma|\right)+\int_{\mathcal{D}_{u, v}}\left|\not \nabla \Phi_{H}\right|(\Sigma \phi \Gamma \\
& +\phi|\not| \Sigma|+\Sigma| \not \nabla \mid) \text {, }
\end{aligned}
$$

and also,

$$
\begin{aligned}
& \sum_{\Phi_{i} \in \Phi_{L}} \int_{\mathcal{N}_{u}(0, v)}\left|\nabla^{2} \Phi_{i}\right|^{2}+\sum_{\Phi_{j} \in \Phi_{S}} \int_{\mathcal{N}_{v}^{\prime}(0, u)} Q^{-1}\left|\nabla^{2} \Phi_{j}\right|^{2} \\
& \leq \sum_{\Phi_{i} \in \Phi_{L}} \int_{\mathcal{N}_{0}(0, v)}\left|\nabla^{2} \Phi_{i}\right|^{2}+\sum_{\Phi_{j} \in \Phi_{S}} \int_{\mathcal{N}_{0}^{\prime}(0, u)} Q^{-1}\left|\nabla^{2} \Phi_{j}\right|^{2} \\
& +\int_{\mathcal{D}_{u, v}}\left|\not{ }^{2} \Phi_{H}\right|\left(\Gamma\left|\not^{2} \Phi\right|+\Phi\left|\not{ }^{2} \Gamma\right|+|\not \nabla \Phi||\not| \Gamma\left|+\Gamma^{2}\right| \not|\Phi|+\Phi \Gamma|\not| \Gamma \mid+\Gamma^{3} \Phi\right) \\
& +\int_{\mathcal{D}_{u, v}}\left|\mathbb{Z}^{2} \Phi_{H}\right|\left(\Sigma \phi \Gamma^{2}+\Gamma \phi|\not \nabla \Sigma|+\Gamma \Sigma|\not \nabla \phi|+\Sigma \phi|\not \nabla \Gamma|+|\not \nabla \phi||\not \nabla \Sigma|\right. \\
& \left.+\phi\left|\not^{2} \Sigma\right|+\Sigma\left|\not^{2} \phi\right|\right) \text {, }
\end{aligned}
$$


and finally,

$$
\begin{aligned}
& \sum_{\Phi_{i} \in \Phi_{L}} \int_{\mathcal{N}_{u}(0, v)}\left|\nabla^{3} \Phi_{i}\right|^{2}+\sum_{\Phi_{j} \in \Phi_{S}} \int_{\mathcal{N}_{v}^{\prime}(0, u)} Q^{-1}\left|\mathbb{X}^{3} \Phi_{j}\right|^{2} \\
& \leq \sum_{\Phi_{i} \in \Phi_{L}} \int_{\mathcal{N}_{0}(0, v)}\left|\mathbb{\nabla}^{3} \Phi_{i}\right|^{2}+\sum_{\Phi_{j} \in \Phi_{S}} \int_{\mathcal{N}_{0}^{\prime}(0, u)} Q^{-1}\left|\nabla^{3} \Phi_{j}\right|^{2}
\end{aligned}
$$

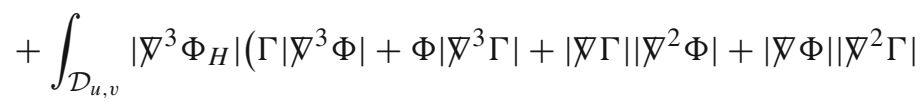

$$
\begin{aligned}
& +\Gamma^{2}\left|\nabla^{2} \Phi\right|+\Gamma \Phi\left|\nabla^{2} \Gamma\right| \\
& \left.+\Gamma|\not| \Gamma|| \not|+\Phi|+\left.\Phi|\not|\right|^{2}+\Gamma^{3}|\not| \Phi\left|+\Phi \Gamma^{2}\right| \not|\nabla \Gamma|+\Gamma^{4} \Phi\right) \\
& +\int_{\mathcal{D}_{u, v}}\left|\mathbb{Z}^{3} \Phi_{H}\right|\left(\Sigma\left|\mathbb{Z}^{3} \phi\right|+\phi\left|\mathbb{Z}^{3} \Sigma\right|+\left|\mathbb{Z}^{2} \phi\right||\not \mathbb{Z} \Sigma|\right. \\
& +\left|\not \mathbb{Z}^{2} \Sigma\right||\not \nabla \phi|+\Sigma \Gamma\left|\mathbb{Z}^{2} \phi\right|+\Sigma \phi\left|\mathbb{Z}^{2} \Gamma\right|
\end{aligned}
$$

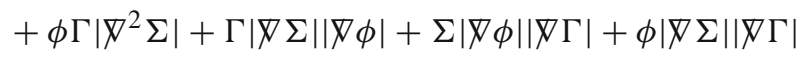

$$
\begin{aligned}
& \left.+\phi \Gamma^{2}|\not \nabla \Sigma|+\Sigma \Gamma|\not| \phi|+\Sigma \phi \Gamma| \not \nabla \Gamma \mid+\Sigma \phi \Gamma^{3}\right) \text {. }
\end{aligned}
$$

As before, we can summarise the previous estimates in the following more concise statement:

Proposition 19 (control of the higher angular derivatives of the components of the Ricci tensor) Suppose that we are given a solution to the CEFEs in Stewart's gauge and that $\mathcal{D}_{u, v}$ is contained in the existence area. Then we have that

$$
\begin{aligned}
& \sum_{\Phi_{i} \in \Phi_{L}} \int_{\mathcal{N}_{u}(0, v)}\left|\mathbb{X}^{m} \Phi_{i}\right|^{2}+\sum_{\Phi_{j} \in \Phi_{S}} \int_{\mathcal{N}_{v}^{\prime}(0, u)} Q^{-1}\left|\mathbb{X}^{m} \Phi_{j}\right|^{2} \\
& \leq \sum_{\Phi_{i} \in \Phi_{L}} \int_{\mathcal{N}_{0}(0, v)}\left|\mathbb{X}^{m} \Phi_{i}\right|^{2}+\sum_{\Phi_{j} \in \Phi_{S}} \int_{\mathcal{N}_{0}^{\prime}(0, u)} Q^{-1}\left|\mathbb{D}^{m} \Phi_{j}\right|^{2} \\
& \quad+\int_{\mathcal{D}_{u, v}}\left|\mathbb{X}^{m} \Phi_{H}\right| \sum_{i_{1}+i_{2}+i_{3}+i_{4}=m}\left(\left|\mathbb{X}^{i_{1}} \Gamma^{i_{2}}\right|\left|\mathbb{X}^{i_{3}} \Gamma\right|\left|\mathbb{X}^{i_{4}} \Phi\right|+\left|\mathbb{X}^{i_{1}} \Gamma^{i_{2}}\right|\left|\mathbb{X}^{i_{3}} \Sigma\right|\left|\mathbb{X}^{i_{4}} \phi\right|\right),
\end{aligned}
$$

where $m=0,1,2,3$.

Using Eqs. (19e) and (19f) we can obtain a similar control over the components $\Phi_{12}$ and $\Phi_{22}$. More precisely, one has that:

Proposition 20 (control of the higher angular derivatives of the "bad" components of the Ricci tensor) Suppose that we are given a solution to the CEFEs in Stewart's gauge and that $\mathcal{D}_{u, v}$ is contained in the existence area. Then we have that 


$$
\begin{aligned}
& \int_{\mathcal{N}_{u}(0, v)}\left|\nabla^{m} \Phi_{12}\right|^{2}+\int_{\mathcal{N}_{v}^{\prime}(0, u)} Q^{-1}\left|\nabla^{m} \Phi_{22}\right|^{2} \\
& \leq \int_{\mathcal{N}_{0}(0, v)}\left|\nabla^{m} \Phi_{12}\right|^{2}+\int_{\mathcal{N}_{0}^{\prime}(0, u)} Q^{-1}\left|\nabla^{m} \Phi_{22}\right|^{2} \\
& +\int_{\mathcal{D}_{u, v}}\left|\mathbb{Z}^{m} \Phi_{22}\right| \sum_{i_{1}+i_{2}+i_{3}+i_{4}=m}\left|\mathbb{X}^{i_{1}} \Gamma^{\prime i_{2}}\right|\left|\mathbb{X}^{i_{3}} \Gamma^{\prime}\right|\left|\mathbb{X}^{i_{4}} \Phi_{22}\right| \\
& +\int_{\mathcal{D}_{u, v}}\left|\mathbb{X}^{m} \Phi_{12}\right| \sum_{i_{1}+i_{2}+i_{3}+i_{4}=m}\left|\mathbb{\nabla}^{i_{1}} \Gamma^{i_{2}}\right|\left|\mathbb{X}^{i_{3}} \Gamma\right|\left|\mathbb{X}^{i_{4}} \Phi\right| \\
& +\int_{\mathcal{D}_{u, v}}\left|\mathbb{Z}^{m} \Phi_{22}\right| \sum_{i_{1}+i_{2}+i_{3}+i_{4}=m}\left|\mathbb{X}^{i_{1}} \Gamma^{i_{2}}\right|\left|\mathbb{X}^{i_{3}} \Gamma\right|\left|\mathbb{X}^{i_{4}} \Phi_{H}^{\prime}\right| \\
& +\int_{\mathcal{D}_{u, v}}\left(\left|\mathbb{X}^{m} \Phi_{12}\right|\left|\mathbb{X}^{i_{1}} \Gamma^{i_{2}}\right|\left|\mathbb{X}^{i_{3}} \Sigma\right|\left|\mathbb{X}^{i_{4}} \phi\right|+\left|\mathbb{X}^{m} \Phi_{22}\right|\left|\mathbb{X}^{i_{1}} \Gamma^{i_{2}}\right|\left|\mathbb{X}^{i_{3}} \Sigma\right|\left|\mathbb{X}^{i_{4}} \phi_{H}^{\prime}\right|\right),
\end{aligned}
$$

where $\Gamma^{\prime}$ does not contain $\tau$ and $\chi, \Phi$ does not contain $\Phi_{00}, \Phi_{H}^{\prime}$ does not contains $\Phi_{22}$ and $\Phi_{00}, \phi$ contains $\phi_{3}$ and $\phi_{4}, \phi_{H}^{\prime}$ contains $\phi_{2}$ and $\phi_{3}$.

Making use of the previous estimates for the Ricci tensor, we can show their boundedness in the truncated diamonds:

Proposition 21 (control of the components of the Ricci tensor in terms of the initial data) Suppose we are given a solution to the vacuum CEFE's in Stewart's gauge arising from data for the CIVP satisfying

$$
\Delta_{e_{\star}}, \Delta_{\Gamma_{\star}}, \Delta_{\Sigma_{\star}}, \Delta_{\Phi_{\star}} \Delta_{\phi_{\star}}<\infty,
$$

with the solution itself satisfying

$$
\begin{aligned}
& \sup _{u, v}\left\|\left\{\mu, \lambda, \alpha, \beta, \epsilon, \rho, \sigma, \tau, \chi, \Sigma_{i}\right\}\right\|_{L^{\infty}\left(\mathcal{S}_{u, v}\right)}<\infty, \\
& \sup _{u, v}\left\|\mathbb{X}\left\{\mu, \lambda, \alpha, \beta, \epsilon, \rho, \sigma, \Sigma_{i}\right\}\right\|_{L^{4}\left(\mathcal{S}_{u, v}\right)}<\infty, \\
& \sup _{u, v}\left\|\mathbb{X}^{2}\left\{\mu, \lambda, \alpha, \beta, \epsilon, \rho, \sigma, \tau, \Sigma_{i}\right\}\right\|_{L^{2}\left(\mathcal{S}_{u, v}\right)}<\infty, \\
& \sup _{u, v}\left\|\mathbb{X}^{3}\left\{\mu, \lambda, \alpha, \beta, \epsilon, \tau, \Sigma_{i}\right\}\right\|_{L^{2}\left(\mathcal{S}_{u, v}\right)}<\infty, \\
& \Delta_{\Phi}(\mathcal{S})<\infty, \quad \Delta_{\Phi}<\infty, \quad \Delta_{\phi}(\mathcal{S})<\infty, \quad \Delta_{\phi}<\infty,
\end{aligned}
$$

on some truncated causal diamond $\mathcal{D}_{u, v_{\bullet}}^{t}$. Then there exists $\varepsilon_{\star}=\varepsilon_{\star}\left(I, \Delta_{e_{\star}}, \Delta_{\Gamma_{\star}}, \Delta_{\Sigma_{\star}}\right.$, $\Delta_{\Phi_{\star}}, \Delta_{\phi}$ ) such that for $\varepsilon_{\star} \leq \varepsilon$ we have

$$
\Delta_{\Phi}<C\left(I, \Delta_{e_{\star}}, \Delta_{\Gamma_{\star}}, \Delta_{\Phi_{\star}}\right) .
$$


Proof We need to control the integration in $\mathcal{D}_{u, v}$ in Propositions 19 and 20. Firstly, we focus on Proposition 19. We need control

$$
\int_{\mathcal{D}_{u, v}}\left|\mathbb{X}^{m} \Phi_{H}\right| \sum_{i_{1}+i_{2}+i_{3}+i_{4}=m}\left(\left|\mathbb{X}^{i_{1}} \Gamma^{i_{2}}\right|\left|\mathbb{X}^{i_{3}} \Gamma\right|\left|\mathbb{X}^{i_{4}} \Phi\right|+\left|\mathbb{W}^{i_{1}} \Gamma^{i_{2}}\right|\left|\mathbb{X}^{i_{3}} \Sigma\right|\left|\mathbb{X}^{i_{4}} \phi\right|\right),
$$

where $\Phi_{H}=\left\{\Phi_{00}, \Phi_{01}, \Phi_{02}, \Phi_{11}, \Phi_{12}\right\}$. We can separate $\left|\mathbb{Z}^{m} \Phi_{H}\right|$ and the summation using the Hölder inequality. In turn, the term $\left|\mathbb{}^{m} \Phi_{H}\right|$ can be controlled as follows:

$$
\left\|\mathbb{Z}^{m} \Phi_{H}\right\|_{L^{2}\left(\mathcal{D}_{u, v}\right)}=\left(\int_{0}^{u} \int_{0}^{v} \int_{S}\left|\mathbb{\nabla}^{m} \Phi_{H}\right|^{2}\right)^{1 / 2} \leq C \Delta_{\Phi} \varepsilon^{1 / 2} .
$$

We observe that as $\Phi$ contains $\Phi_{22}$, we can only control it on $\mathcal{N}_{v}^{\prime}$. Accordingly, we have that

$$
\left\|\not \mathbb{P}^{m} \Phi\right\|_{L^{2}\left(\mathcal{D}_{u, v}\right)} \leq C \Delta_{\Phi}
$$

Next, we need to analyse the $L^{2}$-norm of the summation. Observing that the first term of the summation has a structure similar to that of the Weyl tensor $\Psi$ in vacuum Einstein case, we readily obtain that this term is controlled by

$$
C\left(I, \Delta_{e_{\star}}, \Delta_{\Gamma_{\star}}, \Delta_{\Phi_{\star}}, \Delta_{\Phi}\right) \varepsilon^{1 / 2} .
$$

The second term in the summation can be shown to be less than

$$
C \Delta_{\Phi} \varepsilon^{1 / 2} \sum_{i_{1}+i_{2}+i_{3}+i_{4}=m}\left\|\mathbb{Z}^{i_{1}} \Gamma^{i_{2}} \nabla^{i_{3}} \Sigma \mathbb{Z}^{i_{4}} \phi\right\|_{L^{2}\left(\mathcal{D}_{u, v}\right)} .
$$

Every time we encounter the components $\phi_{0}$ to $\phi_{3}$ and their derivatives, we can control them through the $L^{2}$-norm on the long light cone $\mathcal{N}_{u}$. Moreover, by analogy to $\Phi_{22}$, we control $\phi_{4}$ and its derivatives on the short light cone $\mathcal{N}_{v}^{\prime}$. Hence following the same procedure we can obtain that this norm is less than

$$
C\left(I, \Delta_{e_{\star}}, \Delta_{\Gamma_{\star}}, \Delta_{\Sigma_{\star}}, \Delta_{\Phi_{\star}}, \Delta_{\phi}, \Delta_{\Phi}\right) \varepsilon^{3 / 2} .
$$

In the next step, we consider the terms on the right hand side of the estimate in Proposition 20. The terms

$$
\int_{\mathcal{D}_{u, v}}\left|\mathbb{Z}^{m} \Phi_{12}\right| \sum_{i_{1}+i_{2}+i_{3}+i_{4}=m}\left|\mathbb{\nabla}^{i_{1}} \Gamma^{i_{2}}\right|\left|\mathbb{\nabla}^{i_{3}} \Gamma\right|\left|\mathbb{\nabla}^{i_{4}} \Phi\right|
$$

can be controlled in the same manner as it was done in Proposition 19 and are bounded by

$$
C\left(I, \Delta_{e_{\star}}, \Delta_{\Gamma_{\star}}, \Delta_{\Phi_{\star}}, \Delta_{\Phi}\right) \varepsilon^{1 / 2}
$$


Next, the terms

$$
\int_{\mathcal{D}_{u, v}}\left|\mathbb{\nabla}^{m} \Phi_{22}\right| \sum_{i_{1}+i_{2}+i_{3}+i_{4}=m}\left|\mathbb{\nabla}^{i_{1}} \Gamma^{i_{2}}\right|\left|\mathbb{\nabla}^{i_{3}} \Gamma\right|\left|\mathbb{X}^{i_{4}} \Phi_{H}^{\prime}\right|
$$

can also be controlled because it does not contains the term $\left(\Phi_{22}\right)^{2}$. Moreover, the terms

$$
\sum_{i_{1}+i_{2}+i_{3}+i_{4}=m} \int_{\mathcal{D}_{u, v}}\left|\mathbb{Z}^{m} \Phi_{12}\right|\left|\mathbb{Z}^{i_{1}} \Gamma^{i_{2}}\right|\left|\mathbb{Z}^{i_{3}} \Sigma \| \mathbb{\nabla}^{i_{4}} \phi\right|
$$

can be controlled by

$$
C\left(I, \Delta_{e_{\star}}, \Delta_{\Gamma_{\star}}, \Delta_{\Sigma_{\star}}, \Delta_{\Phi_{\star}}, \Delta_{\phi}, \Delta_{\Phi}\right) \varepsilon^{3 / 2} .
$$

In the case of the term

$$
\int_{\mathcal{D}_{u, v}}\left|\mathbb{\nabla}^{m} \Phi_{22}\right| \sum_{i_{1}+i_{2}+i_{3}+i_{4}=m}\left|\mathbb{\nabla}^{i_{1}} \Gamma^{\prime i_{2}}\right|\left|\mathbb{\nabla}^{i_{3}} \Gamma^{\prime}\right|\left|\mathbb{X}^{i_{4}} \Phi_{22}\right|,
$$

we readily found that it is bounded by

$$
\begin{aligned}
& C\left(I, \Delta_{e_{\star}}, \Delta_{\Gamma_{\star}}, \Delta_{\Phi_{\star}}\right) \int_{0}^{v}\left\|\mathbb{X}^{m} \Phi_{22}\right\|_{L^{2}\left(\mathcal{N}_{v}^{\prime}(0, u)\right)} \sum_{i=0}^{m}\left\|\mathbb{P}^{i} \Phi_{22}\right\|_{L^{2}\left(\mathcal{N}_{v}^{\prime}(0, u)\right)} \\
& \quad \leq C\left(I, \Delta_{e_{\star}}, \Delta_{\Gamma_{\star}}, \Delta_{\Phi_{\star}}\right) \int_{0}^{v} \sum_{i=0}^{m}\left\|\mathbb{P}^{i} \Phi_{22}\right\|_{L^{2}\left(\mathcal{N}_{v}^{\prime}(0, u)\right)}^{2} .
\end{aligned}
$$

Similarly, we also have that

$$
\begin{aligned}
& \sum_{i_{1}+i_{2}+i_{3}+i_{4}=m} \int_{\mathcal{D}_{u, v}}\left|\nabla^{m} \Phi_{22} \nabla^{i_{1}} \Gamma^{i_{2}} \nabla^{i_{3}} \Sigma \nabla^{i_{4}} \phi_{H}^{\prime}\right| \\
& \leq \varepsilon^{3 / 2} C\left(I, \Delta_{e_{\star}}, \Delta_{\Gamma_{\star}}, \Delta_{\Sigma_{\star}}, \Delta_{\Phi_{\star}}, \Delta_{\phi}\right) \int_{0}^{v}\left\|\nabla^{m} \Phi_{22}\right\|_{L^{2}\left(\mathcal{N}_{v}^{\prime}(0, u)\right)}^{2} .
\end{aligned}
$$

Putting together the above estimates in the inequality of Proposition 20 we have that

$$
\begin{aligned}
\sum_{i=0}^{3}\left\|\nabla^{i} \Phi_{22}\right\|_{L^{2}\left(\mathcal{N}_{v}^{\prime}\right)}^{2} \leq & C \Delta_{\Phi_{\star}}+C\left(I, \Delta_{e_{\star}}, \Delta_{\Gamma_{\star}}, \Delta_{\Sigma_{\star}}, \Delta_{\Phi}\right) \varepsilon^{1 / 2} \\
& +C\left(I, \Delta_{e_{\star}}, \Delta_{\Gamma_{\star}}, \Delta_{\Sigma_{\star}}, \Delta_{\Phi_{\star}}, \Delta_{\phi}, \Delta_{\Phi}\right) \varepsilon^{3 / 2} \\
& +\left(C\left(I, \Delta_{e_{\star}}, \Delta_{\Gamma_{\star}}, \Delta_{\Phi_{\star}}\right)+C\left(I, \Delta_{e_{\star}}, \Delta_{\Gamma_{\star}}, \Delta_{\Sigma_{\star}}, \Delta_{\Phi_{\star}}, \Delta_{\phi}\right) \varepsilon^{3 / 2}\right) \\
& \int_{0}^{v} \sum_{i=0}^{m}\left\|\mathbb{P}^{i} \Phi_{22}\right\|_{L^{2}\left(\mathcal{N}_{v}^{\prime}(0, u)\right)}^{2} .
\end{aligned}
$$


Thus, applying the Grönwall's inequality, we obtain that

$$
\Delta_{\Phi} \leq C\left(I, \Delta_{e_{\star}}, \Delta_{\Gamma_{\star}}, \Delta_{\Phi_{\star}}\right)+C\left(I, \Delta_{e_{\star}}, \Delta_{\Gamma_{\star}}, \Delta_{\Sigma_{\star}}, \Delta_{\Phi_{\star}}, \Delta_{\Phi}\right) \varepsilon^{1 / 2}+o\left(\epsilon^{1 / 2}\right) .
$$

Finally, taking $\varepsilon$ small enough we prove the proposition.

The final ingredient in our analysis is the following proposition whose proof is analogous to that of Proposition 17 in Paper I:

Proposition 22 (control of the components of the Rescaled Weyl tensor in terms of the initial data) With the same assumptions in Proposition 21 on some truncated causal diamond $\mathcal{D}_{u, v_{\bullet}}^{t}$. Then there exists $\varepsilon_{\star}=\varepsilon_{\star}\left(I, \Delta_{e_{\star}}, \Delta_{\Gamma_{\star}}, \Delta_{\Sigma_{\star}}, \Delta_{\Phi_{\star}}, \Delta_{\phi_{\star}}\right)$ such that for $\varepsilon_{\star} \leq \varepsilon$ we have

$$
\Delta_{\phi} \leq C\left(I, \Delta_{e_{\star}}, \Delta_{\Gamma_{\star}}, \Delta_{\Phi_{\star}}, \Delta_{\phi_{\star}}\right) .
$$

\section{Concluding the argument}

The estimates obtained in the previous sections can be used in a last slice argument to obtain our main result. The proof is completely analogous to that given in Section 7 in Paper I and is thus omitted.

Theorem 2 Given smooth initial data on $\mathscr{I}^{-} \cup \mathcal{N}_{\star}^{\prime}$ for $0 \leq v \leq I$ as constructed in Lemma 3, there exists $\varepsilon$ such that an unique smooth solution to the vacuum conformal Einstein field equations exists in the region where $0 \leq v \leq I$ and $0 \leq u \leq \varepsilon$ under the coordinate system and $\varepsilon$ can be chosen to depend only on $\Delta_{e_{\star}}, \Delta_{\Gamma_{\star}}, \Delta_{\Sigma_{\star}}, \Delta_{\Phi_{\star}}$ and $\Delta_{\phi_{\star}}$. Moreover, in this region

$$
\begin{aligned}
& \sup _{u, v} \sup _{\Gamma \in\{\chi, \mu, \lambda, \rho, \sigma, \alpha, \beta, \tau, \epsilon\}} \max \left\{\sum_{0}^{1}\left\|\mathbb{X}^{i} \Gamma\right\|_{L^{\infty}\left(\mathcal{S}_{u, v}\right)}, \sum_{i=0}^{2}\left\|\mathbb{X}^{i} \Gamma\right\|_{L^{4}\left(\mathcal{S}_{u, v}\right)}, \sum_{i=0}^{3}\left\|\mathbb{X}^{i} \Gamma\right\|_{L^{2}\left(\mathcal{S}_{u, v}\right)}\right\} \\
& \quad+\sup _{u, v} \max \left\{\sum_{i=0}^{1}\left\|\mathbb{X}^{i} \Sigma_{2}\right\|_{L^{\infty}\left(\mathcal{S}_{u, v}\right)}, \sum_{i=0}^{2}\left\|\mathbb{X}^{i} \Sigma_{2}\right\|_{L^{4}\left(\mathcal{S}_{u, v}\right)}, \sum_{i=0}^{3}\left\|\mathbb{X}^{i} \Sigma_{2}\right\|_{L^{2}\left(\mathcal{S}_{u, v}\right)}\right\} \\
& \quad+\Delta_{\Phi}+\Delta_{\phi} \leq C\left(I, \Delta_{e_{\star}}, \Delta_{\Gamma_{\star}}, \Delta_{\Sigma_{\star}}, \Delta_{\Phi_{\star}}, \Delta_{\phi_{\star}}\right)
\end{aligned}
$$

and

$$
\begin{aligned}
& \sup _{u, v} \max \left\{\sum_{i=0}^{1}\left\|\mathbb{X}^{i} \Sigma_{1,3,4}\right\|_{L^{\infty}\left(\mathcal{S}_{u, v}\right)}, \sum_{i=0}^{2}\left\|\mathbb{X}^{i} \Sigma_{1,3,4}\right\|_{L^{4}\left(\mathcal{S}_{u, v}\right)}, \sum_{i=0}^{3}\left\|\mathbb{X}^{i} \Sigma_{1,3,4}\right\|_{L^{2}\left(\mathcal{S}_{u, v}\right)}\right\} \\
& \quad \leq C\left(I, \Delta_{e_{\star}}, \Delta_{\Gamma_{\star}}, \Delta_{\Sigma_{\star}}, \Delta_{\Phi_{\star}}\right) \varepsilon .
\end{aligned}
$$

Acknowledgements DH was supported by the FCT (Portugal) IF Program IF/00577/2015, PTDC/MATAPL/30043/2017 and Project No. UIDB/00099/2020. PZ acknowledges the support of the China Scholarship Council. 
Open Access This article is licensed under a Creative Commons Attribution 4.0 International License, which permits use, sharing, adaptation, distribution and reproduction in any medium or format, as long as you give appropriate credit to the original author(s) and the source, provide a link to the Creative Commons licence, and indicate if changes were made. The images or other third party material in this article are included in the article's Creative Commons licence, unless indicated otherwise in a credit line to the material. If material is not included in the article's Creative Commons licence and your intended use is not permitted by statutory regulation or exceeds the permitted use, you will need to obtain permission directly from the copyright holder. To view a copy of this licence, visit http://creativecommons.org/licenses/by/4.0/.

\section{A The conformal field equations in the NP formalism}

This appendix serves as a quick reference to our equations. Throughout, we make use of the NP formalism in the conventions used in the book [19] which, in turn, follows the conventions of [20].

\section{A.1 The NP field equations}

Given the NP frame $\left\{l^{a}, n^{a}, m^{a}, \bar{m}^{a}\right\}$, we denote by $D \equiv l^{a} \nabla_{a}, \Delta \equiv n^{a} \nabla_{a}, \delta \equiv$ $m^{a} \nabla_{a}, \bar{\delta} \equiv \bar{m}^{a} \nabla_{a}$ the associated directional derivatives. The commutators are then given by

$$
\begin{aligned}
(\Delta D-D \Delta) \psi & =((\gamma+\bar{\gamma}) D+(\epsilon+\bar{\epsilon}) \Delta-(\bar{\tau}+\pi) \delta-(\tau+\bar{\pi}) \bar{\delta}) \psi, \\
(\delta D-D \delta) \psi & =((\bar{\alpha}+\beta-\bar{\pi}) D+\kappa \Delta-(\bar{\rho}+\epsilon-\bar{\epsilon}) \delta-\sigma \bar{\delta}) \psi, \\
(\delta \Delta-\Delta \delta) \psi & =(-\bar{v} D+(\tau-\bar{\alpha}-\beta) \Delta+(\mu-\gamma+\bar{\gamma}) \delta+\bar{\lambda} \bar{\delta}) \psi, \\
(\bar{\delta} \delta-\delta \bar{\delta}) \psi & =((\bar{\mu}-\mu) D+(\bar{\rho}-\rho) \Delta+(\alpha-\bar{\beta}) \delta-(\bar{\alpha}-\beta) \bar{\delta}) \psi .
\end{aligned}
$$

where $\psi$ is any scalar field. Here and in the following we highlight the terms which vanish in our gauge.

We use the same notation as in Reference [6] to denote the components of Weyl spinor $\Psi_{A B C D}$ and trace-free Ricci spinor $\Phi_{A A^{\prime} B B^{\prime}}$, namely $\left\{\Psi_{0}, \Psi_{1}, \Psi_{2}, \Psi_{3}, \Psi_{4}\right\}$ and $\left\{\Phi_{00}, \Phi_{01}, \Phi_{02}, \Phi_{11}, \Phi_{12}, \Phi_{22}, \Lambda\right\}$. In particular, we have that

$$
\begin{aligned}
\Phi_{00} & \equiv \frac{1}{2} R_{\{a b\}} l^{a} l^{b}, \quad \Phi_{01} \equiv \frac{1}{2} R_{\{a b\}} l^{a} m^{b}, \quad \Phi_{02} \quad \frac{1}{2} R_{\{a b\}} m^{a} m^{b}, \\
\Phi_{11} & \equiv \frac{1}{4} R_{\{a b\}}\left(l^{a} n^{b}+m^{a} \bar{m}^{b}\right), \quad \Phi_{12} \equiv \frac{1}{2} R_{\{a b\}} n^{a} m^{b}, \\
\Phi_{22} & \equiv \frac{1}{2} R_{\{a b\}} n^{a} n^{b}, \quad \Lambda \equiv-\frac{1}{24} R,
\end{aligned}
$$

where $R_{\{a b\}} \equiv R_{a b}-\frac{1}{4} R g_{a b}$.

The structure equations, in turn, have the form

$$
\begin{aligned}
& \Delta \epsilon-D \gamma=\Lambda-\Phi_{11}-\Psi_{2}+\epsilon(2 \gamma+\bar{\gamma})+\gamma \bar{\epsilon}+\kappa \nu-\beta \pi-\alpha \bar{\pi}-\alpha \tau-\pi \tau-\beta \bar{\tau} \\
& \Delta \kappa-D \tau=-\Phi_{01}-\Psi_{1}+3 \gamma \kappa+\bar{\gamma} \kappa-\bar{\pi} \rho-\pi \sigma-\epsilon \tau+\bar{\epsilon} \tau-\rho \tau-\sigma \bar{\tau},
\end{aligned}
$$




$$
\begin{aligned}
& \Delta \pi-D \nu=-\Phi_{21}-\Psi_{3}+3 \epsilon \nu+\bar{\epsilon} \nu-\gamma \pi+\bar{\gamma} \pi-\mu \pi-\lambda \bar{\pi}-\lambda \tau-\mu \bar{\tau}, \\
& \delta \gamma-\Delta \beta=\Phi_{12}-\bar{\alpha} \gamma-2 \beta \gamma+\beta \bar{\gamma}+\alpha \bar{\lambda}+\beta \mu-\epsilon \bar{\nu}-v \sigma+\gamma \tau+\mu \tau, \\
& \delta \epsilon-D \beta=-\Psi_{1}+\bar{\alpha} \epsilon+\beta \bar{\epsilon}+\gamma \kappa+\kappa \mu-\epsilon \bar{\pi}-\beta \bar{\rho}-\alpha \sigma-\pi \sigma, \\
& \delta \kappa-D \sigma=-\Psi_{0}+\bar{\alpha} \kappa+3 \beta \kappa-\kappa \bar{\pi}-3 \epsilon \sigma+\bar{\epsilon} \sigma-\rho \sigma-\bar{\rho} \sigma+\kappa \tau, \\
& \delta v-\Delta \mu=\Phi_{22}+\lambda \bar{\lambda}+\gamma \mu+\bar{\gamma} \mu+\mu^{2}-\bar{\alpha} \nu-3 \beta \nu-\bar{v} \pi+v \tau, \\
& \delta \pi-D \mu=-2 \Lambda-\Psi_{2}+\epsilon \mu+\bar{\epsilon} \mu+\kappa \nu+\bar{\alpha} \pi-\beta \pi-\pi \bar{\tau}-\mu \bar{\rho}-\lambda \sigma \text {, } \\
& \delta \tau-\Delta \sigma=\Phi_{02}-\kappa \bar{\nu}+\bar{\lambda} \rho-3 \gamma \sigma+\bar{\gamma} \sigma+\mu \sigma-\bar{\alpha} \tau+\beta \tau+\tau^{2}, \\
& \bar{\delta} \beta-\delta \alpha=-\Lambda-\Phi_{11}+\Psi_{2}-\alpha \bar{\alpha}+2 \alpha \beta-\beta \bar{\beta}-\epsilon \mu+\epsilon \bar{\mu} \\
& -\gamma \rho-\mu \rho+\gamma \bar{\rho}+\lambda \sigma \\
& \bar{\delta} \gamma-\Delta \alpha=\Psi_{3}-\bar{\beta} \gamma-\alpha \bar{\gamma}+\beta \lambda+\alpha \bar{\mu}-\epsilon \nu-\nu \rho+\lambda \tau+\gamma \bar{\tau}, \\
& \bar{\delta} \epsilon-D \alpha=-\Phi_{10}+2 \alpha \epsilon+\bar{\beta} \epsilon-\alpha \bar{\epsilon}+\gamma \bar{\kappa}+\kappa \lambda-\epsilon \pi-\alpha \rho-\pi \rho-\beta \bar{\sigma}, \\
& \bar{\delta} \kappa-D \rho=-\Phi_{00}+3 \alpha \kappa+\bar{\beta} \kappa-\kappa \pi-\epsilon \rho-\bar{\epsilon} \rho-\rho^{2}-\sigma \bar{\sigma}+\bar{\kappa} \tau, \\
& \bar{\delta} \mu-\delta \lambda=-\Phi_{21}+\Psi_{3}-\bar{\alpha} \lambda+3 \beta \lambda-\alpha \mu-\bar{\beta} \mu-\mu \pi+\bar{\mu} \pi-v \rho+v \bar{\rho}, \\
& \bar{\delta} \nu-\Delta \lambda=\Psi_{4}+3 \gamma \lambda-\bar{\gamma} \lambda+\lambda \mu+\lambda \bar{\mu}-3 \alpha \nu-\bar{\beta} v-v \pi+v \bar{\tau}, \\
& \bar{\delta} \pi-D \lambda=-\Phi_{20}+3 \epsilon \lambda-\bar{\epsilon} \lambda+\bar{\kappa} \nu-\alpha \pi+\bar{\beta} \pi-\pi^{2}-\lambda \rho-\mu \bar{\sigma}, \\
& \bar{\delta} \sigma-\delta \rho=-\Phi_{01}+\Psi_{1}-\kappa \mu+\kappa \bar{\mu}-\bar{\alpha} \rho-\beta \rho+3 \alpha \sigma-\bar{\beta} \sigma-\rho \tau-\bar{\rho} \tau, \\
& \bar{\delta} \tau-\Delta \rho=2 \Lambda+\Psi_{2}-\kappa \nu-\gamma \rho-\bar{\gamma} \rho+\bar{\mu} \rho+\lambda \sigma+\alpha \tau-\bar{\beta} \tau+\tau \bar{\tau} .
\end{aligned}
$$

\section{A.2 Conformal vacuum Einstein field equations}

The rescaled Weyl spinor $\phi_{A B C D}$ is related to the Weyl spinor $\Psi_{A B C D}$ of the NP formalism by

$$
\phi_{A B C D}=\Xi^{-1} \Psi_{A B C D} .
$$

Moreover, $\phi_{A B C D}$ is related to the spinorial counterpart of $d_{a b c d}$ via

$$
d_{A A^{\prime} B B^{\prime} C C^{\prime} D D^{\prime}}=-\phi_{A B C D} \epsilon_{A^{\prime} B^{\prime}} \epsilon_{C^{\prime} D^{\prime}}-\phi_{A^{\prime} B^{\prime} C^{\prime} D^{\prime}} \epsilon_{A B} \epsilon_{C D} .
$$

We denote the components of $\phi_{A B C D}$ by

$$
\begin{gathered}
\phi_{0} \equiv \Xi^{-1} \Psi_{0}, \quad \phi_{0} \equiv \Xi^{-1} \Psi_{0}, \quad \phi_{1} \equiv \Xi^{-1} \Psi_{1}, \quad \phi_{2} \equiv \Xi^{-1} \Psi_{2}, \\
\phi_{3} \equiv \Xi^{-1} \Psi_{3}, \quad \phi_{4} \equiv \Xi^{-1} \Psi_{4} .
\end{gathered}
$$

The spinorial counterpart of the Schouten tensor is

$$
L_{A A^{\prime} B B^{\prime}}=-\Lambda \epsilon_{A B} \epsilon_{A^{\prime} B^{\prime}}+\Phi_{A B A^{\prime} B^{\prime}} .
$$

Finally, we denote the components of the derivative of the conformal factor $\Xi$ by

$$
\Sigma_{1} \equiv D \Xi, \quad \Sigma_{2} \equiv \Delta \Xi, \quad \Sigma_{3}=\delta \Xi=\Xi_{01^{\prime}}, \quad \Sigma_{4}=\bar{\delta} \Xi=\Xi_{10^{\prime}} .
$$




\section{A.2.1 The first conformal Einstein field equation}

The spinorial counterpart of the first conformal Einstein equation, Eq. (2a), is

$$
\nabla_{B B^{\prime}} \nabla_{A A^{\prime}} \Xi=-\Xi \Phi_{A B A^{\prime} B^{\prime}}+s \epsilon_{A B} \bar{\epsilon}_{A^{\prime} B^{\prime}}+\Xi \Lambda \epsilon_{A B} \bar{\epsilon}_{A^{\prime} B^{\prime}}
$$

When we decompose it in terms of the NP null tetrad we obtain

$$
\begin{aligned}
-\Sigma_{1}(\epsilon+\bar{\epsilon})+\Sigma_{4} \kappa+\Sigma_{3} \bar{\kappa}+D \Sigma_{1} & =-\Xi \Phi_{00}, \\
\Sigma_{2}(\epsilon+\bar{\epsilon})-\Sigma_{3} \pi-\Sigma_{4} \bar{\tau}+D \Sigma_{2} & =s+\Xi \Lambda-\Xi \Phi_{11}, \\
-\Sigma_{3}(\epsilon-\bar{\epsilon})+\Sigma_{2} \kappa-\Sigma_{1} \bar{\tau}+D \Sigma_{3} & =-\Xi \Phi_{01}, \\
-\Sigma_{1}(\gamma+\bar{\gamma})+\Sigma_{4} \tau+\Sigma_{3} \bar{\tau}+\Delta \Sigma_{1} & =s+\Xi \Lambda-\Xi \Phi_{11}, \\
\Sigma_{2}(\gamma+\bar{\gamma})-\Sigma_{3} \nu-\Sigma_{4} \bar{v}+\Delta \Sigma_{2} & =-\Xi \Phi_{22}, \\
-\Sigma_{3}(\gamma-\bar{\gamma})-\Sigma_{1} \bar{v}+\Sigma_{2} \tau+\Delta \Sigma_{3} & =-\Xi \Phi_{12}, \\
-\Sigma_{1}(\bar{\alpha}+\beta)+\Sigma_{3} \bar{\rho}+\Sigma_{4} \sigma+\delta \Sigma_{1} & =-\Xi \Phi_{01}, \\
\Sigma_{2}(\bar{\alpha}+\beta)-\Sigma_{4} \bar{\lambda}-\Sigma_{3} \mu+\delta \Sigma_{2} & =-\Xi \Phi_{12}, \\
-\Sigma_{3}(-\bar{\alpha}+\beta)-\Sigma_{1} \bar{\lambda}+\Sigma_{2} \sigma+\delta \Sigma_{3} & =-\Xi \Phi_{02}, \\
\Sigma_{4}(-\bar{\alpha}+\beta)-\Sigma_{1} \mu+\Sigma_{2} \bar{\rho}+\delta \Sigma_{4} & =-s-\Xi \Lambda-\Xi \Phi_{11} .
\end{aligned}
$$

\section{A.2.2 The second conformal Einstein field equation}

The spinorial counterpart of the second conformal Einstein equation, Eq. (2b), is given by

$$
\nabla_{A A^{\prime}} s=\Lambda \nabla_{A A^{\prime}} \Xi-\Phi_{A B A^{\prime} B^{\prime}} \nabla^{B B^{\prime}} \Xi
$$

Its decomposition in terms of the NP null tetrad is given by

$$
\begin{aligned}
-D s & =-\Sigma_{1} \Lambda+\Sigma_{2} \Phi_{00}-\Sigma_{4} \Phi_{01}-\Sigma_{3} \Phi_{10}+\Sigma_{1} \Phi_{11}, \\
-\Delta s & =-\Sigma_{2} \Lambda+\Sigma_{2} \Phi_{11}-\Sigma_{4} \Phi_{12}-\Sigma_{3} \Phi_{21}+\Sigma_{1} \Phi_{22}, \\
-\delta s & =-\Sigma_{3} \Lambda+\Sigma_{2} \Phi_{01}-\Sigma_{4} \Phi_{02}-\Sigma_{3} \Phi_{11}+\Sigma_{1} \Phi_{12} .
\end{aligned}
$$

\section{A.2.3 The third conformal Einstein field equation}

The spinorial counterpart of the third equation is

$$
\begin{aligned}
\nabla_{A A^{\prime}} & \Phi_{B C B^{\prime} C^{\prime}}-\nabla_{B B^{\prime}} \Phi_{A C A^{\prime} C^{\prime}} \\
= & \epsilon_{B C} \bar{\epsilon}_{B^{\prime} C^{\prime}} \nabla_{A A^{\prime}} \Lambda-\epsilon_{A C} \bar{\epsilon}_{A^{\prime} C^{\prime}} \nabla_{B B^{\prime}} \Lambda \\
& -\bar{\phi}_{A^{\prime} B^{\prime} C^{\prime} D^{\prime}} \epsilon_{A B} \nabla_{C}^{D^{\prime}} \Xi-\phi_{A B C D} \bar{\epsilon}_{A^{\prime} B^{\prime}} \nabla_{C^{\prime}}^{D} \Xi .
\end{aligned}
$$

The independent components of this equation can be found to be

$$
\Delta \Phi_{00}-\delta \Phi_{10}+2 D \Lambda=\Phi_{00}(2 \gamma+2 \bar{\gamma}-\mu)-2 \Phi_{10}(\bar{\alpha}+\tau)-2 \Phi_{01} \bar{\tau}
$$




$$
\begin{aligned}
& +2 \Phi_{11} \bar{\rho}+\Phi_{20} \sigma+\Sigma_{3} \bar{\phi}_{1}-\Sigma_{1} \bar{\phi}_{2} \\
& \Delta \Phi_{01}-\delta \Phi_{11}+\delta \Lambda=\Phi_{01}(2 \gamma-\mu)+\Phi_{00} \bar{\nu}+\Phi_{12} \bar{\rho}+\Phi_{21} \sigma \\
& -\Phi_{10} \bar{\lambda}-2 \Phi_{11} \tau-\Phi_{02} \bar{\tau}+\Sigma_{3} \bar{\phi}_{2}-\Sigma_{1} \bar{\phi}_{1}, \\
& \Delta \Phi_{02}-\delta \Phi_{12}=\Phi_{02}(2 \gamma-2 \bar{\gamma}-\mu)+2 \Phi_{12}(\bar{\alpha}-\tau)+2 \Phi_{01} \bar{v} \\
& +\Phi_{22} \sigma-2 \Phi_{11} \bar{\lambda}+\Sigma_{3} \bar{\phi}_{3}-\Sigma_{1} \bar{\phi}_{4}, \\
& \Delta \Phi_{11}-\delta \Phi_{21}+\Delta \Lambda=\Phi_{01} \nu+\Phi_{10} \bar{\nu}+\Phi_{21}(2 \beta-\tau)+\Phi_{22} \bar{\rho}-\Phi_{20} \bar{\lambda} \\
& -2 \Phi_{11} \mu-\Phi_{12} \bar{\tau}+\Sigma_{2} \bar{\phi}_{2}-\Sigma_{4} \bar{\phi}_{3}, \\
& \Delta \Phi_{12}-\delta \Phi_{22}=\Phi_{22}(2 \bar{\alpha}+2 \beta-\tau)+\Phi_{02} \nu+2 \Phi_{11} \bar{\nu}-2 \Phi_{12}(\bar{\gamma}+\mu) \\
& -2 \Phi_{21} \bar{\lambda}+\Sigma_{2} \bar{\phi}_{3}-\Sigma_{4} \bar{\phi}_{4} \\
& D \Phi_{22}-\delta \Phi_{21}+2 \Delta \Lambda=\Phi_{22}(\bar{\rho}-2 \epsilon-2 \bar{\epsilon})+2 \Phi_{21}(\beta+\bar{\pi})+2 \Phi_{12} \pi \\
& -\Phi_{20} \bar{\lambda}-2 \Phi_{11} \mu+\Sigma_{3} \phi_{3}-\Sigma_{2} \phi_{2} \\
& D \Phi_{12}-\delta \Phi_{11}+\delta \Lambda=\Phi_{02} \pi+2 \Phi_{11} \bar{\pi}+\Phi_{12}(\bar{\rho}-2 \bar{\epsilon})+\Phi_{21} \sigma-\Phi_{22} \kappa \\
& -\Phi_{10} \bar{\lambda}-\Phi_{01} \mu-\Sigma_{2} \phi_{1}+\Sigma_{3} \phi_{2}, \\
& D \Phi_{11}-\delta \Phi_{10}+D \Lambda=\Phi_{01} \pi+\Phi_{10}(\bar{\pi}-2 \bar{\alpha})+2 \Phi_{11} \bar{\rho}+\Phi_{20} \sigma-\Phi_{21} \kappa \\
& -\Phi_{12} \bar{\kappa}-\Phi_{00} \mu-\Sigma_{4} \phi_{1}+\Sigma_{1} \phi_{2} \text {, } \\
& D \Phi_{02}-\delta \Phi_{01}=\Phi_{02}(2 \epsilon-2 \bar{\epsilon}+\bar{\rho})+2 \Phi_{01}(\bar{\pi}-\beta)+2 \Phi_{11} \sigma \\
& -2 \Phi_{12} \kappa-\Phi_{00} \bar{\lambda}-\Sigma_{2} \phi_{0}+\Sigma_{3} \phi_{1} \text {, } \\
& D \Phi_{01}-\delta \Phi_{00}=2 \Phi_{01}(\epsilon+\bar{\rho})+\Phi_{00} \bar{\pi}+2 \Phi_{10} \sigma-2 \Phi_{00}(\bar{\alpha}+\beta) \\
& -2 \Phi_{11} \kappa-\Phi_{02} \bar{\kappa}-\Sigma_{4} \phi_{0}+\Sigma_{1} \phi_{1} \text {, } \\
& \delta \Phi_{10}-\bar{\delta} \Phi_{01}=\Phi_{00}(\mu-\bar{\mu})+2 \Phi_{11}(\rho-\bar{\rho})+2 \Phi_{10} \bar{\alpha}+\Phi_{02} \bar{\sigma} \\
& -2 \Phi_{01} \alpha-\Phi_{20} \sigma+\Sigma_{4} \phi_{1}-\Sigma_{3} \bar{\phi}_{1}-\Sigma_{1} \phi_{2}+\Sigma_{1} \bar{\phi}_{2} \text {, } \\
& \delta \Phi_{11}-\bar{\delta} \Phi_{02}+\delta \Lambda=2 \Phi_{02}(\bar{\beta}-\alpha)+\Phi_{01}(\mu-2 \bar{\mu})+\Phi_{12}(2 \rho-\bar{\rho}) \\
& +\Phi_{10} \bar{\lambda}-\Phi_{21} \sigma+\Sigma_{2} \phi_{1}-\Sigma_{3}\left(\phi_{2}+\bar{\phi}_{2}\right)+\Sigma_{1} \bar{\phi}_{3}, \\
& \delta \Phi_{21}-\bar{\delta} \Phi_{12}=2 \Phi_{11}(\mu-\bar{\mu})+\Phi_{22}(\rho-\bar{\rho})+2 \Phi_{12} \bar{\beta}+\Phi_{20} \bar{\lambda} \\
& -2 \Phi_{21} \beta-\Phi_{02} \lambda+\Sigma_{2}\left(\phi_{2}-\bar{\phi}_{2}\right)-\Sigma_{3} \phi_{3}+\Sigma_{4} \bar{\phi}_{3} \text {. }
\end{aligned}
$$

\section{A.2.4 The fourth conformal Einstein field equation}

The spinorial counterpart of the fourth conformal Einstein equation, Eq. (2d), is given by

$$
\nabla_{D C^{\prime}} \phi_{A B C}^{D}=0
$$

Its decomposition in terms of the NP null tetrad is given by

$$
\Delta \phi_{0}-\delta \phi_{1}=-2 \phi_{1}(\beta+2 \tau)+\phi_{0}(4 \gamma-\mu)+3 \phi_{2} \sigma,
$$




$$
\begin{aligned}
\Delta \phi_{1}-\delta \phi_{2} & =2 \phi_{1}(\gamma-\mu)+\phi_{0} \nu+2 \phi_{3} \sigma-3 \phi_{2} \tau, \\
\Delta \phi_{2}-\delta \phi_{3} & =2 \phi_{3}(\beta-\tau)-3 \phi_{2} \mu+2 \phi_{1} \nu+\phi_{4} \sigma, \\
\Delta \phi_{3}-\delta \phi_{4} & =\phi_{4}(4 \beta-\tau)+3 \phi_{2} \nu-2 \phi_{3}(\gamma+2 \mu), \\
D \phi_{1}-\bar{\delta} \phi_{0} & =\phi_{0}(\pi-4 \alpha)+2 \phi_{1}(\epsilon+2 \rho)-3 \phi_{2} \kappa, \\
D \phi_{2}-\bar{\delta} \phi_{1} & =2 \phi_{1}(\pi-\alpha)-\phi_{0} \lambda+3 \phi_{2} \rho-2 \phi_{3} \kappa, \\
D \phi_{3}-\bar{\delta} \phi_{2} & =2 \phi_{3}(\rho-\epsilon)-2 \phi_{1} \lambda+3 \phi_{2} \pi-\phi_{4} \kappa, \\
D \phi_{4}-\bar{\delta} \phi_{3} & =\phi_{4}(\rho-4 \epsilon)+2 \phi_{3}(\alpha+2 \pi)-3 \phi_{2} \lambda .
\end{aligned}
$$

\section{References}

1. Hilditch, D., Valiente Kroon, J.A., Zhao, P.: Revisiting the characteristic initial value problem for the vacuum Einstein field equations (2019). arXiv:1911.00047

2. Luk, J.: On the local existence for the characteristic initial value problem in general relativity. Int. Math. Res. Not. 20, 4625 (2012)

3. Stewart, J.M., Friedrich, H.: Numerical relativity. The characteristic initial value problem. Proc. R. Soc. Lond. A 384, 427 (1982)

4. Friedrich, H.: On the global existence and the asymptotic behaviour of solutions to the EinsteinMaxwell-Yang-Mills equations. J. Differ. Geom. 34, 275 (1991)

5. Kánnár, J.: On the existence of $\mathrm{C}^{\infty}$ solutions to the asymptotic characteristic initial value problem in general relativity. Proc. R. Soc. Lond. A 452, 945 (1996)

6. Kroon, J.A.Valiente: Conformal Methods in General Relativity. Cambridge University Press, Cambridge (2016)

7. Friedrich, H.: On the regular and the asymptotic characteristic initial value problem for Einstein's vacuum field equations. Proc. R. Soc. Lond. A 375, 169 (1981)

8. Friedrich, H.: The asymptotic characteristic initial value problem for Einstein's vacuum field equations as an initial value problem for a first-order quasilinear symmetric hyperbolic system. Proc. R. Soc. Lond. A 378, 401 (1981)

9. Friedrich, H.: On the existence of analytic null asymptotically flat solutions of Einstein's vacuum field equations. Proc. R. Soc. Lond. A 381, 361 (1982)

10. Cabet, A., Chruściel, P.T., Wafo, R.T.: On the characteristic initial value problem for nonlinear symmetric hyperbolic systems, including Einstein equations. (2014)

11. Friedrich, H.: On purely radiative space-times. Commun. Math. Phys. 103, 35 (1986)

12. Friedrich, H.: On static and radiative space-times. Commun. Math. Phys. 119, 51 (1988)

13. Chruściel, P.T., Paetz, T.-T.: Kids like cones. Class. Quantum Grav. 30, 235036 (2013)

14. Chruściel, P.T., Paetz, T.-T.: Solutions of the vacuum einstein equations with initial data on past null infinity. Class. Quantum Grav. 30, 235037 (2013)

15. Friedrich, H.: The taylor expansion at past time-like infinity. Commun. Math. Phys. 324, 263 (2014)

16. Cagnac, F.: Problème de cauchy sur un conoïde caractéristique. Ann. Fac. Sci. Toulouse $2^{e}$ série 2,11 (1980)

17. Cagnac, F.: Problème de cauchy sur un conoïde caractéristique pour des equations quasi-lineaires. Ann. Mat. Pure Appl. 129, 13 (1981)

18. Friedrich, H.: Spin-2 fields on minkowski space near space-like and null infinity. Class. Quantum Grav. 20, 101 (2003)

19. Stewart, J.: Advanced General Relativity. Cambridge University Press, Cambridge (1991)

20. Penrose, R., Rindler, W.: Spinors and Space-Time, vol 1 Two-Spinor Calculus and Relativistic Fields. Cambridge University Press, Cambridge (1984)

21. Friedrich, H.: Cauchy problems for the conformal vacuum field equations in general relativity. Commun. Math. Phys. 91, 445 (1983) 
22. Penrose, R., Rindler, W.: Spinors and Space-Time. Volume 2. Spinor and Twistor Methods in SpaceTime Geometry. Cambridge University Press, Cambridge (1986)

Publisher's Note Springer Nature remains neutral with regard to jurisdictional claims in published maps and institutional affiliations. 